\title{
Genetic characterization, ginsenoside analysis and micropropagation of American ginseng (Panax quinquefolius L.)
}

\author{
Samuel G. Obae \\ West Virginia University
}

Follow this and additional works at: https://researchrepository.wvu.edu/etd

\section{Recommended Citation \\ Obae, Samuel G., "Genetic characterization, ginsenoside analysis and micropropagation of American ginseng (Panax quinquefolius L.)" (2010). Graduate Theses, Dissertations, and Problem Reports. 4638. https://researchrepository.wvu.edu/etd/4638}

This Dissertation is protected by copyright and/or related rights. It has been brought to you by the The Research Repository @ WVU with permission from the rights-holder(s). You are free to use this Dissertation in any way that is permitted by the copyright and related rights legislation that applies to your use. For other uses you must obtain permission from the rights-holder(s) directly, unless additional rights are indicated by a Creative Commons license in the record and/ or on the work itself. This Dissertation has been accepted for inclusion in WVU Graduate Theses, Dissertations, and Problem Reports collection by an authorized administrator of The Research Repository @ WVU. For more information, please contact researchrepository@mail.wvu.edu. 
Genetic characterization, ginsenoside analysis and micropropagation of American ginseng (Panax quinquefolius $\mathbf{L}$.)

\author{
Samuel G. Obae
}

Dissertation submitted to the Davis College of Agriculture, Natural Resources and Design at West Virginia University in partial fulfillment of the requirements for the degree of

Doctor of Philosophy

in

Plant and Soil Science

Todd P. West. Ph.D., Chair

Sven Verlinden, Ph.D.

Daniel Panaccione, Ph.D.

Brent Bailey, Ph.D.

Benjamin Dawson-Andoh, Ph.D

Division of Plant and Soil Sciences

Morgantown, West Virginia

2010

Key words: American ginseng; anthropogenic influence; genetic diversity; HPLC; ginsenosides; root shapes; tissue culture 


\title{
ABSTRACT \\ Genetic characterization, ginsenoside analysis and micropropagation of American ginseng (Panax quinquefolius L.)
}

\begin{abstract}
Samuel G. Obae
American ginseng (Panax quinquefolius L.), is an economically important medicinal plant native to eastern deciduous forests of North America. Like many other medicinal plants worldwide, $P$. quinquefolius is mainly collected from the wild. And because of its growing demand, abundance of wild populations is threatened with over harvesting. In West Virginia, $P$. quinquefolius is available both in cultivation and in the wild. Even though $P$. quinquefolius occurs in all 55 counties in West Virginia, patterns of its harvesting and cultivation vary across the state. As described in chapter 2 , the genetic diversity and population structure of $P$. quinquefolius under two levels of wild harvesting and cultivation intensity in West Virginia was examined using RAPD analysis. It was observed that harvesting pressure decreased genetic diversity whereas cultivation intensity increased genetic diversity. Unlike other medicinal plants that are mostly harvested for their leaf materials, $P$. quinquefolius is harvested for its root material. The root of $P$. quinquefolius is unique because it has a tendency to branch into different morphotypes, and this variation in root shapes influences the perceived quality and hence value of $P$. quinquefolius roots in the market. In chapter 3, the profiles and contents of ginsenosides (bioactive constituents of ginseng) among the three common root morphotypes encountered in $P$. quinquefolius were assessed using HPLC, and a simple economic analysis was conducted to determine if sorting roots to respective morphotypes before selling can leverage returns for the grower. It was found that roots of "man-like" (ML) morphotypes had more total ginsenosides content than those of "bulb or round" (BLB) or "straight or stick" (STK) morphotypes. ML roots exhibited low Rg1/high Re profile whereas roots of BLB and STK morphotypes exhibited both low Rg1/high Re, and high $\mathrm{Rg} 1 /$ low Re profiles. Economic analysis showed that sorting roots to respective morphotypes at the farm level before selling can increase returns even with a modest price mark up for high quality roots (ML) only on condition that the strategy does not result in a price mark down on the other root morphotypes. Given the slow growing nature of ginseng, in vitro culture has been suggested as a quick alternative way of producing ginseng's bioactive components. In chapter 4 , in vitro response and resulting ginsenosides content of explants from the three root morphotypes (ginseng lines) was evaluated. It was found that callus induction response, callus biomass, ginsenoside profiles and total ginsenosides content varied among lines. Correlations between total ginsenosides content of stock plants and callus biomass or total ginsenosides content of callus were positive and highly significant. RADP analysis revealed some genetic differences among root morphotypes. Based on molecular distances of their RAPD profiles, BLB and STK roots grouped into the same cluster and separate from ML roots. However, a specific link between a particular DNA band or banding pattern of roots and a given ginsenosides profile or abundance was not apparent. Overall, these findings will have important implications to the conservation, grading and breeding of $P$. quinquefolius and in vitro production of ginsenosides.
\end{abstract}




\section{Acknowledgements}

I like to thank my main advisor Dr. Todd West for his guidance throughout this study, his energy and enthusiasm for plants is contagious. Many thanks to the members of my committee: Drs. Sven Verlinden, Daniel Panaccione, Brent Bailey, and Benjamin Dawson-Andoh for their valuable input during this research. I am thankful for the gracious times I spent talking about ginseng and plants in general with the late $\mathrm{Mr}$. William Grafton, who was on my committee, his perspectives were valuable in shaping this research.

Also I will like to thank the Division of Plant and Soil Science for the generous financial support for this study and my graduate school. I am deeply thankful to Dr. Louis McDonald and Dr. Hillar Klandorf for allowing me to use their laboratory space and HPLC equipments, and much thanks to Elizabeth Falkenstein for her technical help with HPLC. I am grateful to the growers who donated plant materials for this research, and more importantly the wonderful generosity of ginseng diggers who took time out of their busy schedules to help me locate wild ginseng populations. The treks up and down the mountain terrains which are home to American ginseng enabled me to enjoy the wonderful natural sceneries of the great state of West Virginia, and brought some serene moments and much needed breather from laboratory work. Lastly and most importantly to my wife and kids, thanks a million for your steadfast support and encouragement during the many challenging moments of this research and my graduate school. 


\section{Table of Contents}

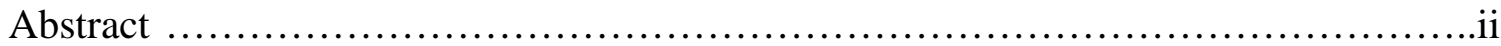

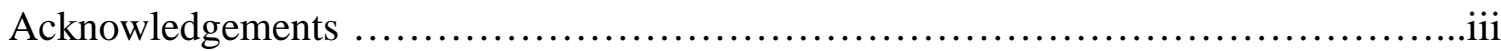

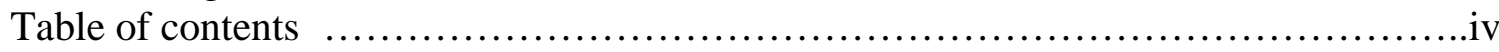

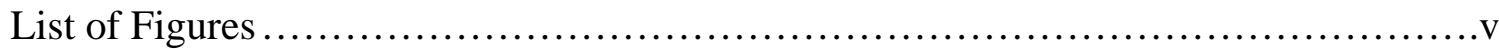

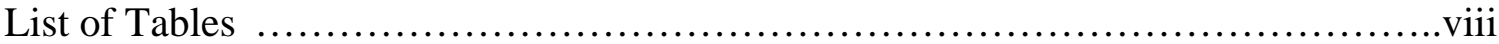

CHAPTER 1

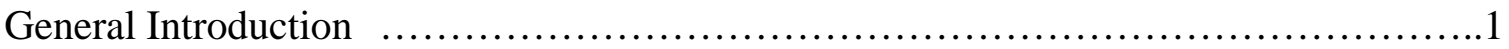

Literature Cited ....................................................................

\section{CHAPTER 2}

Genetic diversity and structure of Panax quinquefolius growing in West Virginia:

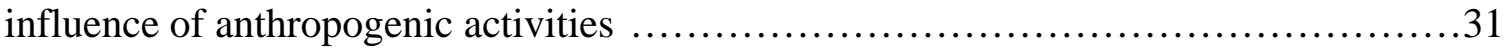

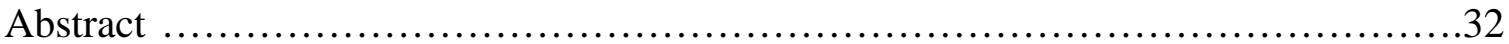

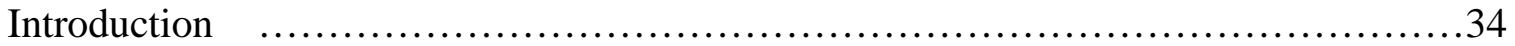

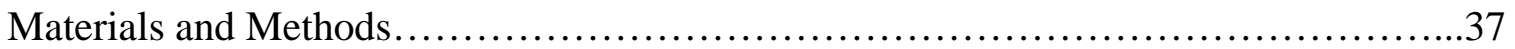

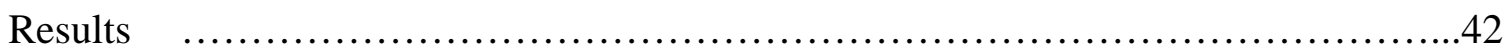

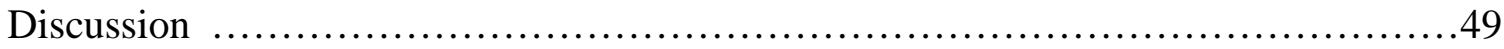

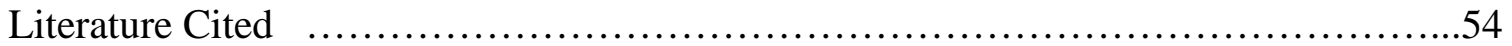

CHAPTER 3

Variation of ginsenosides profiles and content among root morphotypes of American

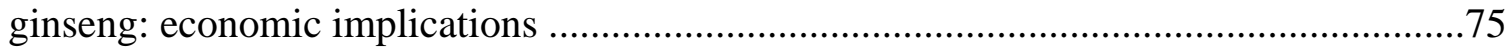

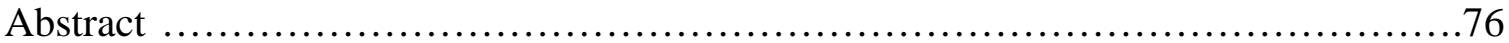

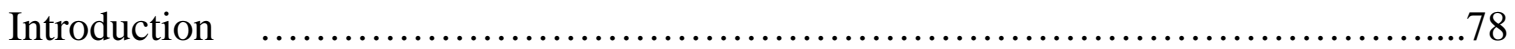

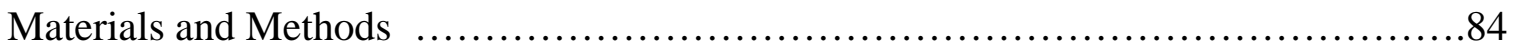

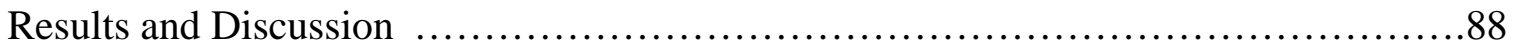

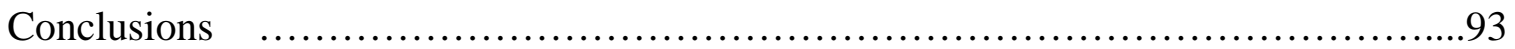

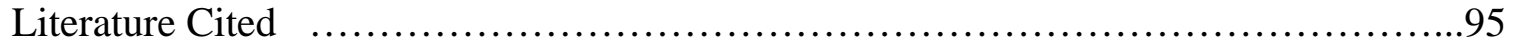

CHAPTER 4

Growth characteristics and ginsenosides production of in vitro tissues of American

ginseng (Panax quinquefolius L.) ，.........................................115

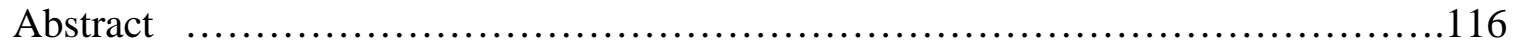

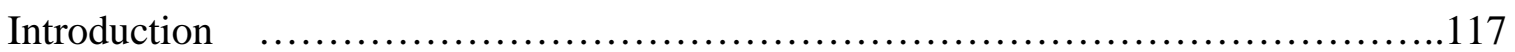

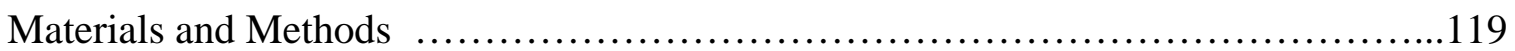

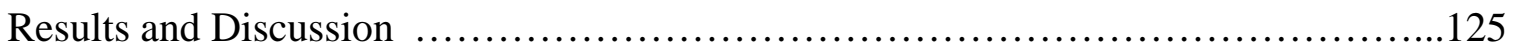

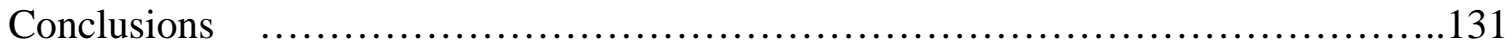

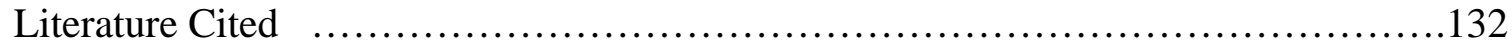

CHAPTER 5

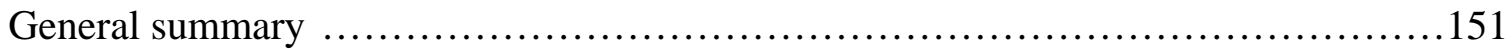

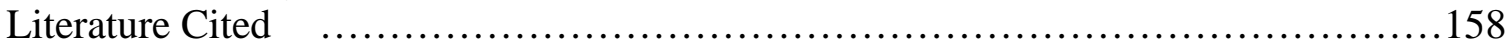




\section{List of Figures}

Figure 1.1. Structures of major ginsenosides in Panax quinquefolius. Adapted from Assinewe et al (2003) ....................................................... 30

Figure 2.1. Map of West Virginia showing Panax quinquefolius cultivation regions and population sites where samples were collected. Region attributes are described in the text. Areas not drawn to scale .......................................61

Figure 2.2. Map of West Virginia showing wild ginseng harvest regions and populations sites where samples were collected. Region attributes are described in the text. Areas not drawn to scale

Figure 2.3. Genomic DNA isolated for Panax quinquefolius leaf samples using Mini plant genomic DNA isolation Kit (Sigma-Aldrich). $1.5 \%$ agarose gel, $\mathrm{M}=1 \mathrm{~kb}$ DNA ladder, $\mathrm{C}=$ negative control, $1-18=$ ginseng leaf samples ................63

Figure 2.4. Reproducibility of band patterns A and B generated by primer UBC-98 in samples $1-18$ of POP_12. $\mathrm{M}=1 \mathrm{~kb}$ DNA marker, $\mathrm{C}=$ negative control. PCR reactions were run in separate days

Figure 2.5. Band patterns generated by primers OPAD-11(A) and OPO-15 (B) in samples 1-10 of POP_13. $\mathrm{M}=1 \mathrm{~kb}$ DNA ladder, arrows indicate polymorphic bands.....65

Figure 2.6. Cluster analysis of populations from groups of American ginseng using UPGMA based on Nei's (1978) unbiased genetic distance with 1000 bootstraps. Population code followed by -C indicates cultivated population, and -W indicates wild population .......................................................... 66

Figure 2.7. Cluster analysis of West Virginia populations using UPGMA based on Nei's (1978) unbiased genetic distances with 1000 bootstraps. Population code followed by -C indicates cultivated population, and -W indicates wild population. Only bootstrap values greater than $60 \%$ are shown

Figure 2.8. Cluster analysis of American ginseng populations from different groups, ecotypes and regions using UPGMA based on Nei's (1978) unbiased genetic distance with 1000 bootstraps ............................................68

Figure 3.1. Different root morphotypes of Panax quinquefolius. A through C; 3 month old roots grown in regular sunshine mix in a greenhouse: A) STK morphotype; B) BLB morphitype; C) ML morphotype; D) 8 year old woods cultivated roots showing all three morphotypes, left to right; STK, BLB and ML morphotypes respectively. The scale bar in $\mathrm{C}$ applies to all 3 month old roots ..................105 
Figure 3.2. HPLC chromatograms of six ginsenosides in P. quinquefolius root samples:

A) mixed standard solution; B) chromatograms showing high $\operatorname{Rg} 1 /$ low Re profile;

C) low $\operatorname{Rg} 1 /$ high Re profile in root samples. Ginsenoside peaks; 1 (Rg1), 2(Re),

3(Rb1), 4(Rc), 5(Rb2), and 6(Rd)

Figure 3.3. Profiles of ginsenoside $\operatorname{Rg} 1$ and Re in roots of P. quinquefolius of three different ages and morphotypes

Figure 3.4. Concentration of ginsenoside Rb1 in roots of $P$. quinquefolius of three different ages and morphotypes.

Figure 3.5. Comparison of total ginsenoside concentration (\% w/w) of P. quinquefolius roots by age and morphotype. Mean concentrations accompanied by same letter are not significantly different at $\alpha=0.05$ following Tukey's multiple comparison

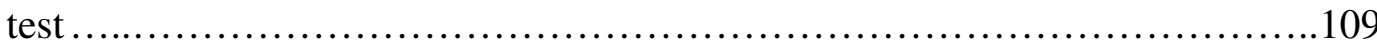

Figure 3.6. Concentration (Adjusted means \pm SEM) of six individual and total ginsenosides of different morphotypes of $P$. quinquefolius roots. Mean concentrations of individual and total ginsenosides accompanied by same letter are not significantly different among morphotypes at $\alpha=0.05$ following Tukey's multiple comparison test

Figure 4.1. Callus induction time of root explants from different ginseng lines cultured in the dark in MS medium with $1.0 \mathrm{mg} \mathrm{l}^{-1} 2$, 4-D and $0.1 \mathrm{mg} \mathrm{l}^{-1}$ Kinetin. Each value is a mean of 6 replicates with 5 explants per replicate. Vertical lines are standard deviations of means

Figure 4.2. Frequency of callus formation on root explants of three American ginseng lines cultured in MS medium with $1.0 \mathrm{mg} \mathrm{l}^{-1}$ 2,4-D and $0.1 \mathrm{mg} \mathrm{l}^{-1}$ Kinetin. Data based on observation of 6 replicates with 5 explants per replicate for each ginseng line 138

Figure 4.3. Callus growth on root explants of different ginseng lines cultured in the dark for $12 \mathrm{wks}$ in MS medium with $1.0 \mathrm{mg} \mathrm{l}^{-1}$ 2,4-D and $0.1 \mathrm{mg} \mathrm{l}^{-1}$ kinetin. Means are for 6 biological replicates with 5 explants per replicate for each ginseng line. Vertical lines are standard deviations of means. Different letters on each bar indicate means are significantly different at $\quad \alpha=0.05$, Tukey's test..........139

Figure 4.4. Relationship between total ginsenosides of stock plants and subsequent dry weight of their callus tissue after 12 wks of culture in the dark in MS medium with $1.0 \mathrm{mg} \mathrm{l}^{-1} 2,4-\mathrm{D}$ and $0.1 \mathrm{mg}^{-1}$ Kinetin. The extreme values are not leverage points; their DFIT values did not exceed $2 \sqrt{ }[(k+1) / n]$, where $k$ is the number of predictors and $n$ the number of data points (Minitab Reference Manual, MINITAB) 
Figure 4.5. Relationship between total ginsenosides of stock roots and that of their callus tissue cultured for $12 \mathrm{wks}$ in the dark in MS medium with $1.0 \mathrm{mg} \mathrm{l}^{-1} 2,4-\mathrm{D}$ and 0.1 $\mathrm{mg} \mathrm{l}^{-1}$ Kinetin. The extreme values are not leverage points; their DFIT values did not exceed $2 \sqrt{ }[(k+1) / n]$, where $k$ is the number of predictors and $n$ the number of data points (Minitab Reference Manual, MINITAB)......................... 141

Figure 4.6. UPGMA based distance relationship between individual plants of $P$. quinquefolius with different root morphotypes (ginseng lines) based on Nei's (1978) unbiased distance of their molecular data from RAPD analysis with 1000 bootstraps. BLB = "bulb or round"; ML = "man-like"; STK = "straight or stick" morphotypes. Only bootstraps values greater than $60 \%$ are indicated

Figure 4.7. UPGMA based distance relationship between three root morphotypes (ginseng lines) of P. quinquefolius based on Nei's (1978) unbiased distance of their molecular data from RAPD analysis with 1000 bootstraps. BLB = "bulb or round"; ML = "man-like"; STK = "straight or stick" morphotypes 143 


\section{List of Tables}

Table 2.1. Population, ecotype, sample size, and population code of populations used in this study

Table 2.2. List of primers used in this study .70

Table 2.3. Summary of within-population genetic diversity. $\mathbf{n}=$ sample size; $\boldsymbol{P}=$ is percent polymorphic loci; $\boldsymbol{H}=$ gene diversity (Nei 1973); $\boldsymbol{I}=$ Shannon's index (Lewontin 1972). Refer to introduction section for groups and regions definition....

Table 2.4. Summary of mean genetic diversity of populations in groups, ecotypes and regions. $\mathbf{n}=$ sample size; $\boldsymbol{P}=$ percent polymorphic loci; $\boldsymbol{H}=$ gene diversity $(\mathrm{Nei}$ 1973); $\boldsymbol{I}$ = Shannon's index (Lewontin 1972), $\boldsymbol{G}_{\boldsymbol{S T}}=$ coefficient of genetic differentiation. Refer to introduction section for group ecotype, and region definitions............................................................ 72

Table 2.5. Mann-Whitney test for differences in mean genetic diversity indices among populations of $P$. quinquefolius in various groups, ecotypes, and regions comparisons

Table 2.6. Summary of analysis of molecular variance (AMOVA) for groups, ecotypes and regions of $P$. quinquefolius using RAPD markers. Significance tests based on 1000 random permutations

Table 3.1. Relative abundance of individual ginsenosides to total content by root morphotype

Table 3.2. Root yield per acre of $P$. quinquefolius under different planting densities...112

Table 3.3. Projected returns from sorting of roots to different morphotypes at farm level prior to selling under current $P$. quinquefolius cultivation system (no cultivars)

Table 3.4. Projected returns of cultivating select cultivar of $P$. quinquefolius

Table 4.1. Rate of callus growth of different ginseng lines cultured in the dark in MS medium with $1.0 \mathrm{mg} \mathrm{l}^{-1} 2,4-\mathrm{D}$ and $0.1 \mathrm{mg} \mathrm{l}^{-1}$ Kinetin

Table 4.2. Pearson correlation among callus weight, total ginsenosides content of callus and stock roots. All correlations were highly significant $(P=0.001)$.

Table 4.3. Ginsenosides content of stock roots and callus tissues of different ginseng lines 
Table 4.4. Ginsenoside contents and profiles of roots and callus tissues of different ginseng lines. Values are means \pm SD of six replicates per line.................147

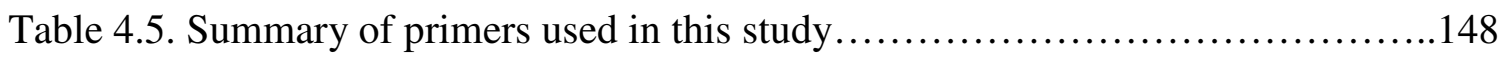

Table 4.6. Distance matrix among individual roots within ginseng lines based on RAPD markers. Distance values based on Nei's (1978) unbiased minimum distance...149

Table 4.7. Distance matrix of three ginseng lines based on RAPD markers. Distance values based on Nei's (1978) unbiased minimum distance.....................150 
CHAPTER 1

General Introduction 
For centuries, people have gathered plants from the wild for use as food and medicine (Schippmann et al. 2002). Even today, hundreds of people mostly in developing countries derive a significant part of their subsistence needs and income from gathered plants and plant products (Iqbal 1993; Walter 2001). Gathering of high value products such as mushrooms and medicinal plants from the wild also continues in developed countries for cultural and economic reasons (Bailey 1999; Jones et al. 2002).

Medicinal plants play a central role, not only as traditional medicines within local cultures, but also as trade commodities that meet the demands of distant markets (Schippmann et al. 2000). Seventy to eighty percent of the world's population in developing countries depends primarily on herbal medicine for basic healthcare needs, mainly due to the long held folklore bequeathed from generation to generation in different cultures and because herbal medicines are cheaper and more easily accessed through traditional healers as opposed to western medicines (Farnsworth et al. 1985; Farnsworth \& Soejarto 1991; Mander et al. 1997; Pei Shengji 2001; Vines 2004; Canter 2005). Studies have also indicated that significant populations of people in developed countries are embracing medicinal practices that involve use of herbal drugs and remedies (Hoareau and DaSilva 1999). For example, over $40 \%$ of US population is using herbal medicines or herbal based dietary supplements and $25 \%$ of the UK populations take herbal medicines regularly (Breevort 1998; Vines 2004; Canter 2005).

Increasing use of herbal products in developed countries is evidenced by their presence in a wide range of retail outlets and their frequent advertisement in popular 
media. Several factors have been attributed to this renaissance, among them; the growing individual interest, rising cost of pharmaceutical based healthcare, increasing success in validating the safety and efficacy of herbal remedies, renewed interest of companies in isolating useful compounds from plants, and legislation improving the status of herbal medicine industry (Iqbal 1993; Leaman 2002). In the US, for instance, the passing of the Dietary Supplement Health and Education Act or "DSHEA" in 1994 has facilitated production and marketing of herbal products and has therefore contributed significantly to their increasing usage (Breevort 1998).

Globally, approximately $8 \%$ of medicinal plants are threatened, however about $75 \%$ of medicinal plant species in use are still collected from the wild (Walter and Gillett 1998; Edwards 2004). The immediate threats to medicinal plants species are anthropogenic activities resulting in habitat loss, habitat degradation and over-harvesting mainly exacerbated by increasing demand for herbal products both regionally and internationally (Cunningham 1993; Hamilton 1997; Kuipers 1997; Ahmad 1998; Lange 1998). However, it has been noted that plants are more likely to go extinct due to over collection than from habitat destruction and population fragmentation alone (Vance 2002).

American ginseng (Panax quinquefolius L.), is among the top 12 commonly used herbs in the US (O'Hara et al. 1998). This perennial herb belongs to the Araliaceae family and is native to eastern deciduous woodlands of North America (Catling et al. 1994). The genus Panax is comprised of eleven species, however, P. quinquefolius and 
P. ginseng (Asian ginseng) are the two species that are commonly utilized for their medicinal qualities (Kitts et al. 2000). Even though the two species grow in similar habitats, they occur in different continents (Pritts 1995). American ginseng was first discovered in Montréal, Canada, by Father Lafitau in 1716 (Nash 1898; Goldstein 1975). Upon its discovery, the French and native Indians started collecting American ginseng roots for export to China where ginseng was extensively used (Nash 1898; Goldstein 1975). In 1750, American ginseng was found in western parts of New England region, and as human population continued moving westward, American ginseng was found in abundance to as far as states west of the Mississippi river (Nash 1898).

Asian ginseng is almost extinct from the wild, therefore the demand for ginseng from Asian consumers is mostly supplied from American ginseng harvested in North America (Pritts 1995). For nearly three centuries, American ginseng has been harvested from the wild and exported to markets in Asia where ginseng use in traditional Chinese medicine (TCM) dates back thousands of years (Duke 1989; Persons 1994; Robbins 1998). This has exerted an enormous pressure on American ginseng populations especially for wild plants since they are highly demanded and command a higher price than cultivated plants (Robbins 1998).

Among the biggest threats to the survival of American ginseng in the wild is overharvesting and habitat destruction. These threats may have been foreseen early in some states such as West Virginia and Virginia since they had put in place, during the last quarter of the $19^{\text {th }}$ century, legislation that regulated ginseng harvest from the wild (Nash 
1898). However, it was not until 1975 that American ginseng was listed as an Appendix II species by CITES (Convention of International Trade in Endangered Species) (Robbins 1998; Sumner 2000). There are 158 national governments who are signed to CITES treaty and are therefore obliged to monitor and control international trade in plants and animals listed in its two main Appendices (Schippmann et al. 2002). Appendix I prohibits trade in wild specimens except for reasons such as scientific research, and Appendix II requires parties to issue export permits that confirm non-detrimental harvest of listed species, and requires importing countries who are parties to CITES to check and monitor permits on incoming material (Schippmann et al. 2002).

Following the listing of American ginseng as an Appendix II species, concerted efforts including enforcement laws governing collection of wild American ginseng were adopted in various state jurisdictions across its range mainly to curb drastic decline of wild populations. American ginseng is designated as "endangered" in Canada, which means that its wild population status are at critical levels and therefore export of wild harvested roots is prohibited (COSEWIC 2001). In the US, some states list American ginseng as "endangered" and thus prohibit collection of wild ginseng within their jurisdictions (Robbins 1998). However, other states designate American ginseng as "threatened", meaning that a decrease of populations in the wild is evident, but has not reached critical levels to warrant prohibition of its collection from the wild (Robbins 1998). 
Those states that allow collection of American ginseng from the wild, however, do require ginseng collectors to purchase a state permit allowing them to harvest wild ginseng within the permit issuing state's jurisdictions (Schumann and Thomas 1993). The permit stipulates the dates within which harvestings is allowed, the age of plants from which roots are to be harvested (mostly three or more prongs), and also requires collectors to keep records of the amount of root they harvested for each collecting season and provide this information to the permit issuing authority upon request (Schumann and Thomas 1993; Robbins 1998; USFWS 2005). The information obtained from these records coupled with research findings help ginseng monitoring authorities to formulate and implement ginseng management and conservation strategies. Despite the strict regulations and guidelines for American ginseng harvest from the wild, illegal harvesting and wanton collection of ginseng outside of stipulated good stewardship guidelines is prevalent and this poses serious threats to the survival of ginseng populations in the wild. A significant proportion of American ginseng exported from the US is still collected from the wild (Pierce 2002). The top three US states producing wild American ginseng are; Kentucky, West Virginia, and Tennessee (Robbins 1998; WVDOF 2008).

Besides wild harvesting, ginseng is also obtained from cultivation. Cultivation of ginseng in North America started in the early 1870s (Nash 1898), however it was not until 1970s, following the listing of American ginseng in CITES that its cultivation really started getting serious consideration among landowners in North America. Most of ginseng cultivation in Canada occurs in the province of British Columbia, whereas in the US, the state of Wisconsin leads in ginseng cultivation (Kitts and $\mathrm{Hu} 2000$; Giblette 
2006; Cheng and Mitchell 2009). Cultivated ginseng supplies the bulk of American ginseng exported from Canada and the US (Pierce 2002). For instance, the annual production of dry roots of cultivated American ginseng in North America in 1999 was over 1000 metric tons, while that of wild ginseng roots was approximately 58 metric tons with a combined market value of about 100 million US dollars (Ren and Chen 1999). Asia still remains the main export destination for American ginseng from the US, with approximately $89 \%$ of the total export sold to Hong Kong, $5 \%$ to Singapore, $4 \%$ to Taiwan and the remaining $2 \%$ to other countries (Robbins 1998).

The effects of over-harvesting on wild American ginseng can be devastating given its biology and is therefore a major concern for plant conservationist. American ginseng exclusively reproduces by seed and has a long reproductive cycle (Lewis 1988). A plant germinates from seed in early spring 18 to 20 months after seeds are released from a mature plant (Hu et al. 1980; Lewis and Zenger 1982; Proctor and Bailey 1987; Pritts 1995). Plants typically do not flower until they are old enough to produce two compound leaves (whorls) (Charron and Gagnon 1991). Flowering may occur during the fourth year; however, it is not until the fifth year or later that a mature fruit is produced (Carpenter and Cottam 1982; Lewis and Zenger 1983; Anderson et al. 1993). The species is mostly self-fertilized (Schlessman 1985); however out-crossing by generalist insects has been reported (Carpenter and Cottam 1982; Lewis and Zenger 1983). Asexual reproduction of American ginseng from rhizome or root fragments is unknown (Lewis 1988). 
Demographic studies have reported declining population sizes of American ginseng in the wild (Robbins 1998). Increasing wild population sizes through "restocking" with cultivated seeds has been encouraged by conservation managers (USFWS 2006). However, there is a growing concern that this practice may affect the existing wild "local" genotypes, as it will result in cross breeding between local and non local populations and will subsequently lead to loss of distinct regional genotypes (Jacobson and Burkhart 2004). Nonetheless, reseeding of ginseng in forests has been a common practice in most places, and much of the wild ginseng likely exists because of deliberate planting of commercial seeds in the past (Jacobson and Burkhart 2004). This raises the question of whether we truly have wild ginseng especially in places frequented by ginseng collectors or surrounded by cultivated ginseng farms.

The bulk of American ginseng sold from West Virginia is collected from wild (WVDOF 2008). American ginseng occurs in all 55 counties in WV; however a significant proportion of harvesting occurs in the southern counties of the state (WVDOF 2008). This is because there are more diggers in the southern part of the state, and for cultural reasons people in these counties have grown up gathering herbs and digging roots (Anonymous 2008). Data from ginseng harvest records in WV over a 30 year period suggests that wild ginseng in WV is experiencing variable harvesting pressure: high harvest pressure, especially among ginseng populations in the southern part of the state, and low harvest pressure on populations in the rest of the state. 
On the other hand, ginseng cultivation is of relatively small scale in West Virginia when compared to a state like Wisconsin. To put this in perspective, in the year 1994 alone, over 2 million pounds of cultivated ginseng was exported to China from Wisconsin (Giblette 2006; Cheng and Mitchell 2009), whereas cultivated ginseng production in WV was 11, 063 pounds over a 30 year period ( 370 pounds/yr) (WVDOF 2008). Not all counties in WV cultivate ginseng, $7 \%$ of the counties have not reported cultivated ginseng production in the last 30 years, and $88 \%$ of all cultivated ginseng production from WV has been from one county during that period (WVDOF 2008). From this data it can be inferred that ginseng cultivation in WV comprises two scales; 1) a small segment of growers who cultivate ginseng on a large scale, and 2) a large segment of growers, mainly consisting of starters who are often less established and cultivate ginseng on a small scale. Under these two different cultivation scales or intensities, the genetic diversity and structure of cultivated ginseng populations could be different.

Generally, during cultivation plant populations can be subjected to different selective pressures which can ultimately change the genetic structures of their populations (Doebley 1989; Hernandez-Verdugo et al. 2001). Collection of seeds from a narrow source for use in establishing cultivated populations can cause genetic drift and bottlenecks that can result in cultivated populations that are significantly diverged from their wild progenitor gene pools (Zohary 2004). Under such circumstances genetic diversity may be relatively low in cultivated populations (Zohary 2004). However, for wild populations that are in close proximity to cultivated areas, the possibility of gene flow back and forth between wild and cultivated populations is likely. In this instance, 
gene flow can reduce genetic isolation and would result in non-distinct wild and cultivated populations (Otero-Arnaiz et al. 2005).

In Chapter 2, the genetic diversity and differentiation of WV populations with that of non-WV populations was compared, genetic differentiations between ginseng ecotypes (wild versus cultivated populations) in WV were assessed, and the effects of harvest pressure and cultivation intensity on unprotected wild and cultivated populations respectively growing in $\mathrm{WV}$ were evaluated. These genetic comparisons were meant to highlight the level of diversity in ginseng populations growing in WV, determine if there are genetic differences between ginseng ecotypes, and examine how anthropogenic activities may be impacting genetic diversity and structure of ginseng populations in WV.

\section{Phtyochemistry of ginseng}

Ginsenosides are the main bioactive components in ginseng. They are available in small quantities and are believed to be responsible for most of ginseng's pharmacological actions (Attele et al. 1999). More than 40 different ginsenosides have been identified, isolated and characterized in ginseng (Teng et al. 2003). The basic structure of ginsenosides is similar, consisting of a gonane steroid nucleus with 17 carbon atoms arranged in four rings with a modified side chain at C-20 (Radad et al. 2006) (Figure 1.1). Based on their structural differences, ginsenosides can be classified into three groups: the protopanaxadiol group (e.g., Rb1, Rb2, Rb3, Rc, Rd, Rg3, Rh2, Rs1), the protopanaxatriol group (e.g., $\mathrm{Re}, \mathrm{Rf}, \mathrm{Rg} 1, \mathrm{Rg} 2, \mathrm{Rh} 1$ ), and the oleanic acid group (e.g., Ro) (Attele et al. 1999; Vanisree et al. 2004). 
The specific mechanisms of pharmacological actions of ginsenosides are yet to be fully elucidated. However, it has been suggested that each ginsenoside may have its own specific tissue-dependent effects and may act independently or in combination with others to produce a biological effect (Ki et al. 1998; Murphy and Lee 2002). For instance ginsenosides $\mathrm{Rg} 1$ and $\mathrm{Rb} 1$ have been reported as efficient neuroprotective agents (Liao 2002), they promote neural growth in vitro, protect neurons against ischemic injury (Zou 2002; Shen and Zhang 2003), enhance learning ability by increasing hippocampal synaptic density (Mook-Jung 2001), and prevent memory loss by increasing the proliferative ability of neural progenitor cells (Wen et al. 1996; Zhang et al. 1998; Shen and Zhang 2003). It has also been reported that ginsenoside Rb1, when topically applied to a burn wound on mice, mediates vascular regeneration by promoting angiogenesis during skin wound repair via stimulation of vascular endothelial growth factor (VEGF) production and elevation of interleukin (IL)-1 $\beta$ resulting from the macrophage accumulation in the burn wound (Kimura et al. 2006).

Other studies have reported ginsenosides $\mathrm{Rb} 1, \mathrm{Rb} 2$ and $\mathrm{Rc}$ as effective anticancer agents as they inhibit tumor angiogenesis and metastasis (Mochizuki et al. 1995; Chang 2003). Ginsenoside Re has been reported to have anti-diabetic and antihyperlipidemic properties (Attele et al. 2002; Xie et al. 2005; Cho et al. 2006). These preliminary findings on medicinal properties of ginsenosides signals a significant potential for their future use in conventional medicine to treat diseases such as cancer, diabetes, amnesia, Parkinson's and Alzheimer's diseases. However rigorous clinical studies will need to be undertaken before ginsenosides are fully integrated into 
conventional medicine (Kitts and $\mathrm{Hu} 2000$ ). Nonetheless, use of ginseng as herbal remedy and supplement will continue to increase.

The root is the most used plant part of ginseng. Variations in content of total and individual ginsenosides have been reported between populations and even among individual roots within a single ginseng population (Smith et al. 1996; Assinewe et al. 2003; Lim et al. 2005). Even unique ginsenoside profiles (chemotypes) that are population specific have been reported in ginseng populations (Schlag and McIntosh 2006). The variations in ginsenoside profiles and contents have been attributed to several factors such as age of the root, growing conditions, and underlying genetics (Smith et al. 1996; Assinewe et al. 2003; Lim et al. 2005; Schlag and McIntosh 2006).

There is a notable bias in preference for roots among ginseng consumers based on root morphotype (Kim 2005). The varied preference significantly influences the way ginseng roots are graded and valued by its consumers (Roy et al. 2003, 2008). American ginseng roots have been classified into 3-5 morphotypes. Sokhansanj et al (1999) classified freshly dug ginseng roots into three distinct morphotypes: Pencil, chunky, and complex. A "Pencil" root resembles a carrot, it has a slender body and lacks major lateral roots; a "chunky" root has 3 to 4 large lateral roots giving a "man" shape to the root; and a "complex" root has a single central body with lateral roots giving a "chicken claw" or "spider" shape to the root. Roy et al (2003) classified dried roots into five categories: chunky, forked, pencil (carrot), spider, and fiber. Following this classification, a "chunky" root is described as bullet or bubble shaped; a "forked" root has a humanoid 
appearance, and a "pencil" root has a main tap root equal to or greater than $5 \mathrm{~cm}$ in length. "Spider" roots have no distinct tap root present or if present is less than $2 \mathrm{~cm}$ in length and have several secondary and tertiary roots radiating from main root. "Fiber" roots comprise of secondary and tertiary roots with diameters of 1-2 mm or less.

The classification by Roy et al (2003) is typical of that employed by wholesalers, who often prune and separate dried roots to attain different grades. For example 'fiber' roots are obtained through pruning of secondary and tertiary roots off of the main roots. A 'spider' root is a very rare morphotype of ginseng and is often times regarded as a variant of a 'chunky' or 'bullet' root. Therefore only three categories are truly representative of the common root morphotypes encountered in ginseng at the farm level; 'Forked' or 'Man-like' (ML) - a branched root exhibiting a humanoid shape; 'Chunky' or 'Bulb' (BLB) - compact, round or bullet shaped root; and 'Pencil/Carrot/Stick' (STK) - slender, elongated tap root without lateral roots (Figure 3.1).

The underlying causes of variations in root morphotypes and consequent effects on ginsenoside profiles have not been extensively studied (Li 1997). Soil texture and bulk density have been attributed to influencing the shape of ginseng root (Li 1997; Park et al. 2005; Roy et al. 2008). It is reported that coarse-textured soils provide little resistance to root growth especially in the seeding year resulting in less desirable STK shaped roots, and that roots grown in loam soil are shorter and rounder than those grown in sand soil (Roy et al. 2008). Increasing bulk density (BD) has also been reported to result in roots that are shorter, fatter, with wide mid-sections, and chunkier (desirable 
type) (Roy et al. 2008). Whereas BD and soil texture may play a role in determining ginseng root shape, the effects of underlying genetics cannot be discounted, especially in light of the fact that effects of genotype on root morphology have been documented on Radish (Rhapanus sativus L.) (Iwata et al. 2004; Tsuro et al. 2007).

Despite numerous reports on ginsenoside content and profiles in ginseng root, there has been less focus on relative abundance of individual ginsenosides among different root morphotypes. There are no known cultivars of ginseng (Schluter and Punja 2002), and this is largely attributed to general lack of breeding direction for medicinal plants (Hong et al. 2005). Long held biased consumer preference for different root morphotypes coupled with research findings about pharmacological properties of individual ginsenosides will help set breeding targets for ginseng. In chapter 3 , the influence of underlying genotypes on root morphotype was assessed, and frequency distribution of the three morphotypes (ML, BLB, and STK) among ginseng population was tabulated. Also the concentrations of six main ginensosides ( $\mathrm{Rg} 1, \mathrm{Re}, \mathrm{Rb} 1, \mathrm{Rc}, \mathrm{Rb} 2$, and $\mathrm{Rd}$ ) among the three root mophotypes were compared, with particular attention drawn to variations of $\operatorname{Rg} 1, \operatorname{Re}$ and $\mathrm{Rb} 1$ ginsenosides profiles. Lastly, the potential economic implications for the grower who may like to sort his harvested roots to different morphotypes prior to selling them as a way to leverage return for his crop were evaluated.

\section{In vitro production of ginsenosides}

Field cultivation of American ginseng is a time-consuming and labor intensive process. It takes 5-7 years from seedling to the final harvestable root, and during that time a lot of 
care must be given to plants since plant growth is exposed to various pathogens and pests (Proctor 1996). With the increasing demand for ginseng, relying on wild collection and field cultivation alone will not satisfy the growing need for ginseng and ginseng products. The bioactive components of ginseng produced in vitro have been shown to be similar to those produced from native roots growing in natural environments, however their levels vary with cell lines and culture conditions (Jhang et al. 1974). Use of in vitro culture has therefore been looked at as a potential alternative and a more efficient way to produce ginseng and its bioactive components (Wu and Zhong 1999). With in vitro culture, production is more controllable with regard to quality and quantity, since cell lines with desirable productivity can be selected (Wu and Zhong 1999).

Various physical factors that affect in vitro growth and development of plant cells and tissues have been reported, among them: physical form of medium, $\mathrm{pH}$, temperature, and light (Borkird et al. 1986). For solid medium; $\mathrm{pH}$ range is typically between 5.0 and 6.0 , the growth chamber is usually maintained between $20^{\circ} \mathrm{C}$ and $30^{\circ} \mathrm{C}$, and light intensity of less than 1000 lux is preferred (Borkird et al. 1986). Light requirement can vary from species to species, for ginseng, usually callus and cell suspensions are maintained in the dark and light is not required for cell growth and saponin production (Choi et al. 1994a). Other factors that may influence in vitro organogenic response of explants include; size of the explant, physiological stage of the donor plant, and the organ that serves as an explant source (Thorpe 1981; Choi et al. 1994a). The composition of culture medium has also been observed to influence callus organogenesis and ginsenoside production in $P$. ginseng (Bonfill et al. 2002), and influence of genotype on the growth and development 
of tissue cultures and ginsenoside metabolism has been reported in the genus Panax (Mathur et al. 2003).

There are only few studies on tissue culture of $P$. quinquefolius reported in literature compared to numerous publications on other species of the genus Panax such as $P$. ginseng and $P$. notoginseng. The few studies reported on $P$. quinquefolius tissue culture have basically focused on optimizing in vitro conditions for plantlet regeneration (Wang 1990; Punja et al. 2004; Zhou and Brown 2005). Thus far, minimal research has been done to elucidate the genetic influence on organogenesis and ginsenoside accumulation of $P$. quinquefolius in vitro tissues. American ginseng roots exhibit different morphological characteristics and this may be a consequence of underlying genetics since this species has been reported to be genetically heterogeneous (Assinewe et al. 2003). In chapter 4, the genetic diversity among the three morphotypes (BLB, ML, and STK) of American ginseng roots that were obtained from a single population was assessed, and the response of their explant tissues to in vitro callus initiation, growth and subsequent ginsenosides accumulation was evaluated. 


\section{Literature Cited}

Ahmad, B. 1998. Plant exploration and documentation in view of land clearing in Sabah. In Nair, M.N.B. and N. Ganapathi, eds., Medicinal Plants. Cure for the 21st Century. Biodiversity Conservation and Utilization of Medicinal Plants. pp. 161162. Serdang, Malaysia.

Anderson, R. C., J. E. Armstrong, P. K. Benjamin, and J. S. Fralish. 1993. The ecology and biology of Panax quinquefolium L. (Araliaceae) in Illinois. The American Midland Naturalist 129: 357-372.

Anonymous. 2008. American Ginseng and the Idea of the Commons. In Tending the Commons: Folklife and Landscape in Southern West Virginia. The Library of the Congress. http://memory.loc.gov/ammem/collections/tending/essay1a.html. Accessed on April 302008.

Assinewe, V.A., B.R. Baum, D. Gagnon, J.T. Arnason. 2003. Phytochemistry of wild populations of Panax quinquefolius L. (North American ginseng). Journal of Agricultural and Food Chemistry 51: 4549-4553.

Attele, A. S., J.A. Wu, C.S. Yuan. 1999. Ginseng pharmacology: multiple constituents and multiple actions. Biochememical Pharmacology 58: 1685-1693.

Attele, A. S., Y.P. Zhou, J.T. Xie, J.A. Wu, L. Zhang, L. Dey, W. Pugh, P.A. Rue, K.S. Polonsky, and C.S. Yuan. 2002. Antidiabetic effects of Panax ginseng berry extract and the identification of an effective component. Diabetes 51: 1851-1858.

Bailey, B. 1999. Social and economic impacts of wild harvested products. PhD dissertation, Forest Resource Science, West Virginia University, Morgantown, West Virginia, USA. 
Bonfill, M., M. C. Rosa, J. Palazon, M. T. Pinol, and C. Morales. 2002. Influence of auxins on organogenesis and ginsenoside production in Panax ginseng calluses. Plant, Tissue and Organ Culture 68: 73-78.

Borkird, C., C. H. Choi, and R. Sung. 1986. Effects of 2, 4 dichlorophenoxyacetic acid on the expression of embryogenic program in carrot. Plant physiology 81: $1143-1146$.

Brevoort P. 1998. The blooming U.S. botanical market: a new overview. Herbalgram 44: 33-46.

Canter, P.H. 2005. Bringing medicinal plants into cultivation. Focus on Alternative and Complementary Therapy 10: 167-168.

Carpenter, S. G., and G. Cottam. 1982. Growth and reproduction of American ginseng (Panax quinquefolius) in Wisconsin, USA. Canadian Journal of Botany 60: 2692-2696.

Catling, P. M., E. Haber, and E. Small. 1994. Poorly known economic plants of Canada: ginseng_Panax quinquefolius L. Canadian Botanical Association Bulletin 27: $70-72$.

Chang, Y.S., E.K. Seo, C. Gyllenhaal, and K.I. Block. 2003. Panax ginseng: a role in cancer therapy?. Integrative Cancer Therapies 2: 13-33.

Charron, D., and D. Gagnon. 1991. The demography of northern populations of Panax quinquefolium (American ginseng). Journal of Ecology 79: 431-445.

Cheng, L. and P.D. Mitchell. 2009. Status of the Wisconsin Ginseng Industry. Department of Agricultural and Applied Economics, University of Wisconsin-Madison, Madison, Wisconsin, USA. 
Cho, W.C.S., W-S. Chung, S.K.W. Lee, A.W.N. Leung, C.H.K. Cheng, and K.K.M. Yue. 2006. Ginsenoside Re of Panax ginseng possesses significant antioxidant and antihypelipidemic efficacies in streptozotocin-induced diabetic rats. European Journal of Pharmacology 550: 173-179.

Choi, K. T., I. O. Ahn, and J. C. Park. 1994a. Production of ginseng saponin in tissue culture of Ginseng (Panax ginseng C. A. Mayer). Russian Journal of Plant Physiology 41: 784-788.

Cunningham, A.B. 1993. African medicinal plants. Setting priorities at the interface between conservation and primary healthcare. People and Plants Working Paper 1. UNESCO, Paris, France.

COSEWIC. 2001. Assessment and Update Status Report of the American ginseng (Panax quinquefolius) in Canada.

Doebley, J. 1989. Isozymic evidence and evolution of crop plants. In: Soltis E.D. and Soltis P.M. (eds), Isozymes in Plant Biology. Dioscorides, Portland, Oregon, pp $165-191$.

Duke, J. 1989. Ginseng: A concise handbook. Reference Publication, Inc. Algonac. Mississippi. USA.

Edwards, R. 2004. No remedy in sight for herbal ransack. New Scientist.181: 10-11. Farnsworth, N.R. and D.D. Soejarto 1991. Global importance of medicinal plants. In Akerele, O., V. Heywood and H. Synge, (eds). Conservation of medicinal plants. pp. 25-51, Cambridge, UK, University Press.

Farnsworth N.R., O. Akerele and A.S. Bingel. 1985. Medicinal plants in therapy. Bulletin of the World Health Organization 63: 965-981. 
Giblette, J. 2006. The Role of Cultivation in Conserving Medicinal Plants. In Call et al., 2006. Mending the Web of Life: Chinese Medicine and Species Conservation. Published by the International Fund for Animal Welfare (IFAW) and the Foundation for Education and Research on Botanicals, American Herbal Products Association (AHPA-ERB).

Goldstein B. 1975. Ginseng: Its history, dispersion, and folk tradition. American Journal of Chinese Medicine 3: 223-234.

Hamilton, A.C. 1997. Threats to plants: an analysis of Centres of Plant Diversity. In Conservation into the 21 st Century. Proceedings of $4^{\text {th }}$ International Botanic Gardens Conservation Congress (ed. D. H. Touchell and K. W. Dixon), pp. 309322. Kings Park and Botanic Garden, Perth, Australia.

Hernandez-Verdugo, S., R. Luna-Reyes, and K. Oyama. 2001. Genetic structure and differentiation of wild and domesticated populations of Capsicum annuum from Mexico. Plant Systematics and Evolution 226: 129-142.

Hu, Y. S., L. Rudenberg, and P. D. Tredici. 1980. Studies of American Ginseng. Rhodora 82: 627-638.

Hoareau, L. and E.J. DaSilva. 1999. Medicinal plants: a re-emerging health aid. Electronic Journal of Biotechnology 2(2): 56-70.

Hong, Y. Q. D., A.J. Lau, C. L. Yeo, X. K. Liu, C. R. Yang, H. L. Koh, and Y. Hong. 2005. Genetic diversity and variation of saponin contents in Panax notoginseng root from a single farm. Journal of Agricultural and Food Chemistry. 53: 8460 8467. 
Iqbal, M. 1993. International trade in non-wood forest products. An overview. FAO, Rome.

Jacobson, M. and E. Burkhart. 2004. Opportunities from Ginseng Husbandry in Pennsylvania, Penn State College of Agricultural Sciences, University Park, Pennsylvania, USA.

Jhang, J.J., E.J. Staba, and J.Y. Kim. 1974. American and Korean ginseng tissue culture: growth, chemical analysis, and plantlet production. In Vitro 9: 253-259.

Jones, E.T., R.J. McLain, and J. Weigand. 2002. Nontimber forest products in the United States. - University of Kansas Press, Lawrence, Kansas, USA.

Ki, Y.N., S.-R. Ko, and K.-J. Choi. 1998. Relationship of saponin and non-saponin for the quality of ginseng. Journal of Ginseng Research 22: 272-283.

Kim, H., B. Sung, J. Park, C. Sim, Y. Kim, and S. Lee. 2005. Application of neutron radiography and tomography for analysis of root morphology of growing ginseng. Proceedings of the International Symposium on Research Reactor and Neutron Science - In Commemoration of the $10^{\text {th }}$ Anniversary of HANARO- Daejeon, Korea.

Kimura, Y., M. Sumiyoshi, K. Kawahira, and M. Sakanaka. 2006. Effects of ginseng saponins isolated from Red ginseng roots on burn wound healing in mice. British Journal of Pharmacology 148: 860-870.

Kitts, D.D. and C. Hu. 2000. Efficacy and safety of ginseng. Public Health Nutrition 3: 473-485.

Kitts, D.D., A.N. Wijewickreme, and C. Hu. 2000. Antioxidant properties of a North American ginseng extract. Molecular Cellular Biochemistry 203: 1-10. 
Kuipers, S.E. 1997. Trade in medicinal plants. In Medicinal plants for forest conservation and healthcare, vol. 11. Non-Wood Forest Products (ed. G. Bodeker, K. K. S. Bhat, J. Burley and P. Vantomme), pp. 45-59. FAO, Rome, Italy.

Lange, D. 1998. Europe's medicinal and aromatic plants: their use, trade and conservation: an overview. TRAFFIC International, Cambridge, UK.

Leaman, D. 2002. Medicinal plants. Briefing notes on the impacts of domestication/cultivation on conservation. Paper for the "Commercial captive propagation and wild species conservation" workshop, 7-9.12.2001, Jacksonville. (Unpublished report).

Lewis, W. H. 1988. Re-growth of a Decimated Population of (Panax quinquefolium) in a Missouri Climax Forest. Rhodora 90: 1-5.

Lewis, W., and V. Zenger. 1982. Population dynamics of American ginseng (Panax quinquefolium) Araliaceae. American Journal of Botany 69 (9): 1483-1490.

Lewis, W., and V. Zenger. 1983. Breeding systems and fecundity in the American ginseng, Panax quinquefolium (Araliaceae). American Journal of Botany 70 (3): $466-468$.

Li, T.S.C. 1997. Effects of seedling depth and of soil texture texture on seedling emergence and root shape of American ginseng. Korean Journal of Ginseng Research 21: 115-118.

Liao, B., H. Newmark, and R. Zhou. 2002. Neuroprotective effects of ginseng total saponin and ginsenoside $\mathrm{Rb} 1$ and $\operatorname{Rg} 1$ on spinal code neurons in vitro. Experimental Neurology 173: 224-234. 
Lim, W., K.W. Mudge, and F.Vermeylen. 2005. Effects of population, age, and cultivation methods on ginsenoside content of wild American ginseng (Panax quinquefolium). Journal of Agricultural and Food Chemistry 53: 8498-8505.

Mander, M., J. Mander, and C. Breen. 1997. Promoting the cultivation of indigenous plants for markets. Experiences from KwaZulu-Natal, South Africa. In Leakey, R.R.B., A.B. Temu, M. Melnyk \& P. Vantomme, eds., Domestication and commercialization of non-timber forest products in agroforestry systems. FAO, Rome, Italy.

Mathur, A., A.K. Mathur, R.S. Sangwan, A. Gangwar, and G.C. Uniyal. 2003. Differential morphogenetic response, ginsenoside metabolism and RAPD patterns of three Panax species. Genetic Resource and Crop Evolution 50: 245-252.

Murphy, L. L. and T.J. Lee. 2002 Ginseng, sex behavior and nitric oxide. Annals of the New York Academy of Science 962: 372-377.

Mochizuki, M., Y.C. Yoo, K. Matsuzawa, K. Sato, I. Saiki, and S. Tonooka. 1995. Inhibitory effect of tumor metastasis in mice by saponins, ginsenoside-Rb2, 20(R)- and 20(S)- ginsenoside-Rg3 of red ginseng. Biological and Pharmaceutical Bulletin 18: 1197-1202.

Mook-Jung, I., H.S Hong, J.H. Boo, K.H. Lee, S.H. Yun, M.Y. Cheong, I. Joo, K. Huh, and M.W. Jung. 2001. Ginsenoside Rb1 and Rg1 improve spatial learning and increase hippocampal synaptophysin level in mice. Journal of Neuroscience Research 63: 509-515.

Nash, G.V. 1898. American ginseng: Commercial history, protection, and cultivation. Government printing press, Washington D.C. USA. 
O’Hara, M., D. Kiefer, K. Farrell, and K. Kemper. 1998. A review of 12 commonly used medicinal herbs. Archives of Family Medicine 7: 523-53.

Otero-Arnaiz A., A. Casas, J.L. Hamrick, and J. Cruse-Sanders. 2005. Genetic variation and evolution of Polaskia chichipe (Cactaceae) under domestication in the Tehuacán Valley, central Mexico. Molecular Ecology 14: 1603-1611.

Park, H., B.D. Lee, and J.-M. Lee. 2005. Effect of soil condition on the root contraction of ginseng. Acta Horticulturae 676: 155-160.

Persons, W. S. 1994. American ginseng: Green Gold. Bright Mountain Books, Inc. Asheville, North Carolina, USA.

Pierce, A. R. 2002. American ginseng (Panax quinquefolius): Biology, ecology, trade, social issues, and prospects for conservation. Case study of the Rainforest Alliance Sustainable Botanicals Program. New York, New York, USA.

Punja, Z.K., M. Feeney, C. Schluter, and T. Tautorus. 2004. Multiplication and germination of somatic embryos of American ginseng derived from suspension cultures and biochemical and molecular analyses of plantlets. In Vitro Cellular and Developmental Biology-Plant 40 (3): 329-338.

Pritts, K. D. 1995. How to find, grow, and use America's forest gold. Stackpole Books. Mechanicsburg, Pennsylvania, USA.

Proctor, J.T.A. 1996. Ginseng: Old crop, new directions. In: Janick J (ed) Progress in new crops. ASHS Press, Arlington, Virginia, USA.

Proctor J. T. A. and W. G. Bailey. 1987. Ginseng: Industry, botany, and culture. Horticulture Reviews 9: 187-236. 
Radad, K., G. Gille, L. Liu, and W.D. Rausch. 2006. Use of ginseng in medicine with emphasis on neurodegenerative disorders. Journal of Pharmacological Science 100: $176-186$.

Ren, G. and F. Chen. 1999. Simultaneous Quantification of Ginsenosides in American Ginseng (Panax quinquefolium) Root Powder by Visible/Near-Infrared Reflectance Spectroscopy. Journal of Agricultural and Food Chemistry 47: 27712775.

Robbins, C. S. 1998. American ginseng: The root of North America's medicinal herb trade. TRAFFIC North America Report. Number B347, Washington, D.C. USA.

Roy, R.C., R. Grohs, and R.D. Reeleder. 2003. A method for the classification by shape of dried roots of ginseng (Panax quinquefolius L.). Canadian Journal of Plant Science 83: 955-958.

Roy, R.C., B.R. Ball Coelho, R.D. Reeleder, A.J. Bruin, R. Grohs, P.H. White, and B.B. Capell. 2008. Effect of planting bed shape, mulch and soil density on root yield and shape in North American ginseng (Panax quinquefolius L.). Canadian Journal of Plant Science 88 (5): 937-949.

Schippmann, U., D.J. Leaman, and A.B. Cunningham 2002. Impact of Cultivation and Gathering of Medicinal Plants on Biodiversity: Global Trends and Issues. FAO, Rome. Italy.

Schlag, E.M. and M.S. McIntosh. 2006. Ginsenoside content and variation among and within American ginseng (Panax quinquefolius L.) populations. Phytochemistry 67: $1510-1519$. 
Schlessman, M. 1985. Floral biology of American ginseng (Panax quinquefolium). Bulletin of the Torrey Botanical Club 112 (2): 129-133.

Schluter, C. and Z. K. Punja. 2002. Genetic diversity among natural and cultivated field populations and seed lots of American ginseng (Panax quinquefolius L.) in Canada. International Journal of Plant Science 163 (3): 427-439.

Schumann, D. R. and M. G. Thomas. 1993. Income opportunities in special forest products: Self help suggestions for rural entrepreneurs. Agricultural Information Bulletin (666). U.S. Department of Agriculture, Washington, D.C. USA.

Shen, L.and J. Zhang. 2003. Ginsenoside Rg1 increases ischemia-induced cell proliferation and survival in the dentate gyrus of adult gerbils. Neuroscience Letters 344: 1-4.

Shengji, P. 2001. Ethnobotanical approaches of traditional medicine studies: some experiences from Asia. Pharmaceutical Botany 39: 74-79.

Smith, R.G., D. Caswell, A. Carriere, and B. Zielke. 1996. Variation in the ginsenoside content of American ginseng, Panax quinquefolius L., roots. Canadian Journal of Botany 74: 1616-1620.

Sokhansanj, S., W.G. Bailey, Y.P. Guo, K.B. van Dalfsen, and A.L. Oliver. 1999. Physical characteristics of North American ginseng (Panax quinquefolius L.) root. Canadian Society of Agricultural Engineering 41: 239-246.

Sumner, J. 2000. The natural history of medicinal plants. Timber Press, Portland, Oregon.USA. 
Teng, R., C. Ang, D. McManus, D. Armstrong, S. Mau, and A. Bacic. 2003. Regioselective acylation of ginsenoside by Novozyme 435. Tetrahedron Letters 44: 5661-5664.

Thorpe, T. A. 1981. Plant tissue culture: Methods and applications in agriculture. Academic Press, University of Calgary, Alberta, Canada.

Tsuro, M., K. Suwabe, N. Kubo, S. Matsumoto, and M. Masashi. 2007. Maping of QTLs controlling root shape and red pigmentation in radish, Raphanus sativum L. Breeding Science 58: $55-61$.

USFWS. 2005. Ginseng Findings. http://www.fws.gov/international/pdf/2005ginsengfinding.pdf. Accessed February10, 2006.

USFWS. 2006. Convention Permit Applications for Ginseng Harvested in 2006. Washington, D.C. USA.

Vance, N.C. 2002. Ecological considerations in sustainable use of wild plants. In: Jones E.T., McLain R.J.and Weigand J. (eds) Nontimber Forest Products in the United States. pp. 151-162. University Press of Kansas, Lawrence, Kansas, USA.

Vanisree, M., C.Y. Lee, S.F. Lo, S. M. Nalawade, C.Y. Lin, and H.S. Tsay. 2004. Studies on the production of some important secondary metabolites from medicinal plants by plant tissue cultures. Botanical Bulletin of Academia Sinica 45: $1-22$.

Vines, G. 2004. Herbal harvests with a future: towards sustainable sources for medicinal plants, Plantlife International. www.plantlife.org.uk. Accessed January 8, 2008. 
Walter, S. 2001. Non-wood forest products in Africa. A regional and national overview. FAO, Rome, Italy.

Walter, K.S. and H.J. Gillett 1998. 1997 IUCN Red List of threatened plants. IUCN, Switzerland.

Wang, A. 1990. Callus induction and plant regeneration of American ginseng. HortScience 25 (5): 57-572.

Wen, T. C., H. Yoshimura, S. Matsuda, J.H. Lim, and M. Sakanaka. 1996. Ginseng root prevents learning disability and neuronal loss in gerbil with 5-minute forebrain ischemia. Acta Neuropathologica 91: 15-22.

Wu, J. and J.J. Zhong. 1999. Production of ginseng and its bioactive components in plant cell culture: Current technological and applied aspects. Journal of Biotechnology 68: 89-99.

WVDOF. 2008. http://www.wvforestry.com/ginseng.cfm?menucall=ginseng. Accessed April 22, 2008.

Xie, J-T., S.R. Mehendale, X. Li, R. Quigg, X. Wang, C-Z. Wang, J.A. Wu, H.H. Aung, P.A. Rue, G.I. Bell, and C-S. Yuan. 2005. Anti-diabetic effect of ginsenoside Re in ob/ob mice. Biochimica et Biophysica Acta 1740 (3): 319-325.

Zhang, B., S. Matsuda, J. Tanaka, N. Tateishi, N. Maeda, T-C. Wen, H. Peng, and M.Sakanaka. 1998. Ginsenoside Rb1 prevents image navigation disability, cortical infarction, and thalamic degeneration in rats with focal cerebral ischemia. Jornal of Stroke and Cerebrovascular Diseases 7 (1): 1-9. 
Zhou, S. and D.C.W. Brown. 2005. High efficiency plant production of North American ginseng via somatic embryogenesis from cotyledon explants. Plant Cell Report 25: $166-173$.

Zohary, D. 2004. Unconscious selection and the evolution of domesticated plants. Economic Botany 58 (1): 5-10.

Zou, K., S. Zhu, M. R. Meselhy, C. Tohda, and K. Komatsu. 2002. Dammarene type saponins from Panax japonicus and their neutrite outgrowth activity in SK-N-SH cells. Journal of Natural Products 65: 1288-1292. 


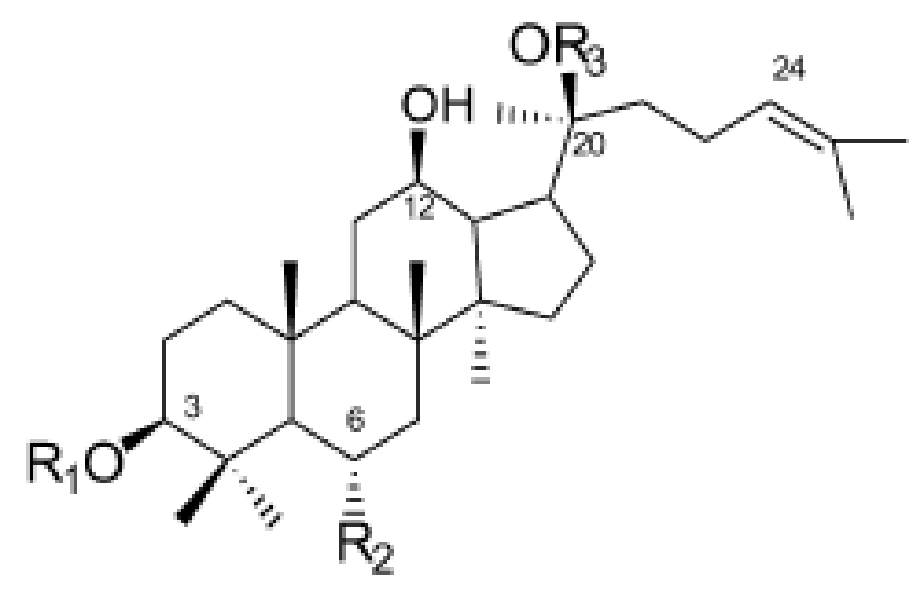

\begin{tabular}{|c|c|c|c|}
\hline Ginsenoside & $\mathrm{R} 1$ & $\mathrm{R} 2$ & R3 \\
\hline \multicolumn{4}{|c|}{ Protopanaxadiol } \\
\hline $\mathrm{Rb} 1$ & Glc- ${ }^{2}$ Glc- & $\mathrm{H}$ & Glc- ${ }^{6}$ Glc- \\
\hline $\mathrm{Rb} 2$ & Glc- ${ }^{2}$ Glc- & $\mathrm{H}$ & Ara(pyr)- ${ }^{6}$ Glc- \\
\hline $\mathrm{Rc}$ & Glc- ${ }^{2}$ Glc- & $\mathrm{H}$ & Ara(fur) $-{ }^{6}$ Glc- \\
\hline $\mathrm{Rd}$ & Glc- ${ }^{2}$ Glc- & $\mathrm{H}$ & Glc- \\
\hline \multicolumn{4}{|c|}{ Protopanaxatriol } \\
\hline $\operatorname{Rg} 1$ & $\mathrm{H}$ & Glc-O- & Glc- \\
\hline $\mathrm{Re}$ & $\mathrm{H}$ & Rha- ${ }^{2}$ Glc-O- & Glc- \\
\hline
\end{tabular}

Figure 1.1. Structures of major ginsenosides in Panax quinquefolius. Adapted from Assinewe et al (2003). 


\section{CHAPTER 2}

Genetic diversity and structure of Panax quinquefolius growing in West Virginia:

influence of anthropogenic activities 


\begin{abstract}
Using random amplified polymorphic DNA (RAPD) markers, genetic variation and structure of wild and cultivated populations of American ginseng growing in West Virginia were assessed and compared to those of populations from other states (Pennsylvania and Wisconsin). Effects of cultivation intensity and harvest pressure on genetic diversity of ginseng populations in West Virginia were also evaluated. Eight primers were used, generating a total of 98 discernable and reproducible bands of which $84(85.71 \%)$ bands were polymorphic. Overall mean genetic diversity measures were lower in West Virginia populations compared to populations from Pennsylvania and Wisconsin. Among West Virginia populations, mean genetic diversity indices were higher in cultivated than in wild populations. With regard to cultivation intensity, populations from a region with low cultivation intensity showed lower levels of genetic diversity (percent polymorphism $(P)=29.93 \%$, gene diversity $(H)=0.0948$, and Shannon's index $(I)=0.1609)$ compared to populations from a region with high cultivation intensity $(P=60.60 \%, H=0.2593, I=0.3243)$, these diversity measures were significantly different $(P<0.05$, Mann-Whitney test $)$. Analysis of molecular variance (AMOVA) further revealed that in cultivated populations $53.68 \%$ and $42.11 \%$ of the total genetic variation was attributed to within and among population differentiation in regions respectively, and a small (4.20\%) but significant variation was found among regions. Significantly higher population genetic diversity evidenced in cultivated populations from a region with high cultivation intensity could be attributed to large scale growers sourcing seeds from a broad seed source to meet their cultivation scale therefore increasing gene flow. Whereas, lower diversity levels among populations in regions with
\end{abstract}


less cultivation intensity could be due to small scale growers probably starting their crop from a narrow seed source, mostly from seeds collected from a single population in the wild or purchased from a single source. With regard to harvest pressure on wild populations, those from low harvest pressure regions had higher genetic diversity indices $(P=33.33 \%, H=0.1172$, and $I=0.1743$ ) compared to populations from high harvest pressure regions $(P=28.27 \%, H=0.1019, I=0.1513)$, however diversity measures were not significantly different $(P>0.05$, Mann-Whitney test). AMOVA further showed no significant genetic differentiation between the two harvest pressure regions $(P=0.7918)$, but genetic differentiation within populations (48.37\%) and among populations (54.10\%) were significant $(P<0.001)$. Even though there was lack of significant differences in genetic diversity indices between wild populations from the two regions, lower diversity indices in high harvest pressure regions indicate that harvesting pressure could reduce genetic diversity of ginseng populations. These results would have useful implications for conservation of this important medicinal plant. 


\section{Introduction}

The immediate threats to medicinal plants species are anthropogenic activities resulting in habitat loss, habitat degradation and over harvesting mainly exacerbated by increasing demand for herbal products both regionally and internationally (Cunningham 1993; Hamilton 1997; Kuipers 1997; Ahmad 1998; Lange 1998). About 75\% of medicinal plant species in use are still collected from the wild (Canter 2005), and it has been noted that plants are more likely to go extinct due to over collection than from habitat destruction and population fragmentation alone (Vance 2002).

Cultivation of medicinal plants has begun to emerge as a common practice mainly as a response to declining wild sources. Increase in cultivation of medicinal plants is particularly motivated by possible economic gains stemming from growing demand for herbal products globally. However, during cultivation, plant populations can be subjected to different selective pressure which can ultimately change the genetic structures of their populations (Doebley 1989; Hernandez-Verdugo et al. 2001). Collection of seeds from a narrow source for use in establishing a cultivated population can cause genetic drift and bottlenecks that can result in populations that have diverged significantly from their wild progenitor gene pools (Zohary 2004). Under such circumstance genetic diversity may be relatively low in cultivated populations (Zohary 2004). However, for wild populations that are in close proximity to their cultivated relatives, the possibility of gene flow back and forth between wild and cultivated populations is likely and that will cause reduction in genetic isolation therefore resulting in non-distinct wild and cultivated populations (Otero-Arnaiz et al. 2005). 
American ginseng (Panax quinquefolius L.), is among the top 12 commonly used herbs in the US (O'Hara et al. 1998) and one of the most widely used medicinal herbs in the world (Christensen et al. 2006). Its growing popularity among consumers worldwide has led to extensive collection from the wild and increasing cultivation within and outside of its native range (Cruse-Sanders and Hamrick 2004; Christensen et al. 2006). West Virginia is at the core of American ginseng's native range, and collection of American ginseng from the wild has been a part of the folklore of West Virginians for hundreds of years (Anonymous 2008). Out of the 19 states authorized to export ginseng, West Virginia ranks second in wild ginseng production (Anonymous 2008; WVDOF 2008). Even though American ginseng grows in the wild throughout the state, more than half of the wild harvested roots come from eight counties in the state's southwestern corner (Anonymous 2008; WVDOF 2008).

Ginseng cultivation in West Virginia however is of relatively small scale when compared to a state like Wisconsin. Cultivated ginseng exported to China from Wisconsin in 1994 alone was over 2 million pounds (Giblette 2006; Cheng and Mitchell 2009), whereas total cultivated ginseng production from WV was 11,063 pounds over a 30 year period ( $\sim 370$ pounds/yr) (WVDOF 2008 ). Not all counties in West Virginia cultivate ginseng, $7 \%$ of the counties have not reported cultivated ginseng production in the last 30 years, and $88 \%$ of all cultivated ginseng production from West Virginia has been from one county during that period (WVDOF 2008). 
Based on ginseng production data in West Virginia (WVDOF 2008), it is apparent that wild ginseng populations occurring in areas where ginseng harvest is permitted face two levels of harvest pressure: high harvest pressure especially in southern parts of the state where collection of wild ginseng is dominant, and low harvest pressure in the rest of the state where ginseng harvest is low to moderate. Likewise, ginseng cultivation intensity in West Virginia can be categorized into two scales: high cultivation intensity regions mainly comprised of a small segment of well established growers, and low cultivation intensity regions mostly comprised of a large segment of growers basically starting out and often less established.

Genetic variation and structure under these varied levels of harvest pressure is relatively unexplored, and little is known about how the cultivation process has impacted genetic variation and structure in American ginseng populations in West Virginia. Genetic variation is a valuable resource from which populations derive short-term adaptation to environmental stochasticities and long-term evolutionary changes (Ellstrand and Elam 1993), and is a good reservoir from which breeding varieties can be derived. The amount and structure of genetic variation in cultivated populations is shaped in part by how genetic material is obtained and how it is transferred from one cultivated generation to the next (Abbo et al. 2003; Miller and Schaal 2006). Cultivated crop species that are started with seeds obtained from a few wild plants often result in cultivated populations that are less diverse than their wild relatives (founder effect) (Abbo et al. 2003). The goal of this study was to compare genetic diversity of West Virginia ginseng populations to populations in other states, and assess genetic diversity 
and population structure of American ginseng populations under varied levels of anthropogenic influences.

\section{Materials and methods}

Study species- Panax quinquefloius L. is a long-lived perennial herb with palmate compound leaves and a fleshy tap root. The species belongs to Araliaceae family and is native to eastern deciduous woodlands of North America, from southern Ontario and Quebec in Canada, along the Appalachian Mountains, south to Georgia and west to Minnesota and Oklahoma (Catling et al. 1994). It is allotetraploid with 2n $=48$ chromosomes (Hu et al. 1980). Plants typically do not flower until they are old enough to produce two leaf whorls (Charron and Gagnon 1991). Flowering may occur during the fourth year; however, it is not until the fifth year or later that a mature fruit is produced (Carpenter and Cottam 1982; Lewis and Zenger, 1983; Anderson et al., 1993). The species is mostly self-fertilized (Schlessman, 1985) however, outcrossing by generalist insects has been reported (Carpenter and Cottam 1982; Lewis and Zenger 1983). A mature plant may produce 30 to 40 fruits on each inflorescence, and seeds usually undergo a dormancy period of 18 to 20 months before germinating in early spring of the second growing season (Hu et al. 1980; Lewis and Zenger 1982; Proctor and Bailey 1987; Pritts 1995). Asexual reproduction of American ginseng from rhizome or root fragments is unknown (Lewis 1988).

Plant materials - Leaf samples from 468 plants in 26 populations of wild and cultivated P. quinquefolius were collected in 2007 and 2008 from West Virginia, Pennsylvania, and 
Wisconsin (Table 2.1). Leaf samples from cultivated populations were donated by growers, whereas samples of wild populations were collected from the wild with the help of ginseng diggers in various regions. At least 9 randomly selected plants within a 50 meter radius of a contiguous patch of ginseng plants were sampled. Upon collection, individual leaf materials were wrapped separately in moist paper towels then placed in Ziploc $^{\circledR}$ bags (S.C. Johnson \& Sons, Inc., Racine, WI) and transported back to the laboratory within 24 hours. Samples from Wisconsin were collected by the grower following the same procedure as all other samples and were shipped to West Virginia University horticulture research laboratory using FedEx next day delivery. Once in the laboratory, leaf samples were surface rinsed with distilled water then immediately frozen in liquid nitrogen and stored in Ziploc ${ }^{\circledR}$ bags at $-80^{\circ} \mathrm{C}$ until DNA extraction.

To assess the effect of cultivation intensity and harvest pressure on genetic diversity of ginseng populations, samples in West Virginia were collected from populations in specifically demarcated regions reflecting different cultivation intensity and harvest pressure. Regions were demarcated based on ginseng harvest records obtained from West Virginia Department of Forestry (WVDOF 2008) for the period between 1978 and 2006. Regarding cultivation intensity, two regions were categorized. Region 1 (WVCR1), low cultivation intensity, was mainly comprised of small scale ginseng farms typically less than 1 acre of land under ginseng cultivation with reported ginseng production averaging less than 10 pounds per year; and region 2 (WVCR2), high cultivation intensity, was comprised of large scale ginseng farms reportedly producing on average 300 pounds or more per year over a 30 year period (Figure 2.1). With regard to 
harvest pressure on wild ginseng populations two regions were categorized. These regions are located in areas where harvesting of wild ginseng is allowed. Region 1 (WVWR1), a low harvest pressure region, where ginseng collection averages less than 500 pounds per year; and region 2 (WVWR2), a high harvest pressure region, where ginseng collection averages over 500 pounds per year over a 30 year period (Figure 2.2).

DNA isolation- Approximately $20 \mathrm{mg}$ of leaf material was placed in $1.5 \mathrm{ml}$ micro centrifuge tube and ground with a sterile disposable plastic pestle (Fisher Scientific, Pittsburgh, PA). DNA extraction was done using a GenElute Plant Genomic DNA Mini Prep Kit (Catalog no. G2N70, Sigma-Aldrich, St. Louis, MO) following the manufacturer's instructions. Quality of isolated genomic DNA was assessed by running $10 \mu$ of DNA template through $1.5 \%$ agarose gel in $0.5 x$ TBE buffer (Tris-base, Boric acid, and EDTA). Gels were stained with ethidium bromide (EtBr), then visualized on electronic UV transilluminator (ULTRA-LŪM, Inc., Claremont, CA) and digitally photographed with Canon Powershot G6 camera (Canon USA, Inc.). A single high molecular genomic DNA band devoid of a smear indicated intact high quality genomic DNA suitable for subsequent RAPD analysis (Figure 2.3). Purity of genomic DNA was also assessed based on 260/280 $\mathrm{nm}$ absorbance ratios obtained from a spectrophotometer (GeneQuat, Pharmacia, LKB Biochrom, England). A ratio of $>1.5$ was considered good quality genomic DNA sample for use in subsequent analysis. For those samples whose DNA absorbance ratios were $<1.5$ or whose quality appeared poor by electrophoresis, DNA extraction was repeated. For each sample isolated genomic DNA was quantified 
and diluted to $20 \mathrm{ng} / \mu \mathrm{l}$ with milliQ water and stored at $-20^{\circ} \mathrm{C}$ until when needed for RAPD analysis.

Primer selection - Twenty-nine, 10-bp primers were initially selected from a list of those that were reported as successful in RAPD analysis of $P$. quinquefolius in previously published studies. Primers UBC-98, UBC-203, UBC-297 were selected from Bai et al. (1997); primers, OPD-03, OPD-05, OPH-04, OPH-05, OPO-15, OPU-10, OPU-15 were from Boehm et al. (1999); primers UBC-06, UBC-18, UBC-81, UBC-164, UBC-177, UBC-210, UBC-227, UBC-262, UBC-326, UBC-398, UBC-419, UBC-464, UBC-497 were from Schluter and Punja (2002), and primers OPAD-01, OPAD-02, OPAD-11, OPAD-15, OPN-02 , OPN-19 were from Lim (2004). These primers were prescreened for polymorphism and reproducibility in amplification products using one sample each from all 26 populations. Eight of the 29 primers exhibited high polymorphic and reproducibile products and were therefore selected for further RAPD analysis of all other samples (Table 2.2). All primers were purchased from Operon Biotechnologies (Huntsville, AL).

PCR amplification and gel electrophoresis - RAPD amplification conditions were initially optimized following a series of trials in which DNA template amount and primer concentrations were varied to determine conditions that produced discernible and reproducible bands (Figure 2.4). Upon establishment of optimal PCR conditions, the rest of the samples were amplified twice with duplicate amplifications run on separate days. A HotStarTaq ${ }^{\circledR}$ Master Mix Kit ( Catalog no. 203443, Qiagen ${ }^{\circledR}$, Germantown, MD) was 
used for PCR amplification with each $25 \mu$ l mixture containing: $12.5 \mu$ l Hotstart mix, $11 \mu \mathrm{l}$ of RNase free $\mathrm{H}_{2} \mathrm{O}, 0.5 \mu \mathrm{l}$ of primer $(0.2 \mu \mathrm{M})$, and $1 \mu \mathrm{l}$ of DNA template $(\sim 20 \mathrm{ng})$. All PCR amplifications were done on a single thermo cycler (GeneAmp ${ }^{\circledR}$ PCR System 9700, Applied Biosystems) with a PCR thermocycling program based on the protocol of Schluter and Punja (2002) with some modifications. The initial PCR cycle included heating the mixture for $15 \mathrm{~min}$ at $94^{\circ} \mathrm{C}$ to activate the HotStarTaq DNA polymerase, as recommended by the manufacture, then $10 \mathrm{~min}$ at $36^{\circ} \mathrm{C}$, and $2 \mathrm{~min}$ at $72^{\circ} \mathrm{C}$. Subsequent 46 cycles included denaturation at $94^{\circ} \mathrm{C}$ for $30 \mathrm{~s}$, annealing at $36^{\circ} \mathrm{C}$ for $1 \mathrm{~min}$, and elongation at $72^{\circ} \mathrm{C}$ for $1 \mathrm{~min}$. In the final cycle primer elongation at $72^{\circ} \mathrm{C}$ was extended for $10 \mathrm{~min}$. Ten micro litres of amplified fragments were loaded into $1.5 \%$ agarose gels alongside 1 kb DNA ladder (Promega, Madison, WI) and separated electrophoretically in 0.5x TBE buffer at $105 \mathrm{~V}$ for 40 min. Gels were stained with EtBr, visualized with electronic UV transilluminator, and digitally photographed.

Data analysis - RAPD bands were scored as present (1) or absent (0) in a binary matrix for each primer. Monomorphic bands across all samples and bands below $250 \mathrm{bp}$ or above 3000 bp were omitted from subsequent genetic statistical analysis. Bands outside of this range (250 - $3000 \mathrm{bp})$ are considered unreliable for RAPD analysis (Stewart and Porter 1995). To estimate genetic diversity and population structure, various parameters were calculated using different software programs for population genetic analysis. Percent polymorphic loci $(P)$, Shannon information index $(I)$ (Lewontin 1972), Nei's (1973) gene diversity $(H)$ and genetic differentiation $\left(G_{S T}\right)$ were calculated using POPGENE ver. 1.31 software (Yeh et al. 1997). Statistical significance of genetic 
diversity measures among different levels of comparisons was determined using MannWhitney test in MINITAB release 13.20 (MINITAB, State College, PA, USA).

Hierarchical population genetic structure (two or three levels) and fixation index $\left(\Phi_{\mathrm{ST}}\right)$ values were determined by Analysis of Molecular Variance (AMOVA) using Arlequin ver. 3.11 software (Excoffier et al. 2005). Significance tests were performed using 1000 permutations. AMOVA uses the phenotypic distance to describe how the variance is partitioned among and within populations, and tests for the significance against the null hypothesis of no population structure (Excoffier et al. 1992; Stewart and Excoffier 1996). AMOVA was based on Euclidean squared distance matrix which was constructed using AMOVA-PREP software (Miller 1997). To illustrate genetic relationships between different groups or population levels, a cluster analysis (UPGMA) was used to generate dendrograms based on Nei's (1978) unbiased distance using TFPGA software program (Miller 1998) with 1000 bootstrap replications.

\section{Results}

RADP polymorphism - Eight primers used in RAPD analysis of 26 populations (468 samples) generated a total of 98 distinct loci (average 12.25 loci per primer) of which 84 were polymorphic $(85.71 \%)$ - an average of 10.5 polymorphic loci per primer (Table 2.2). The 84 polymorphic bands were clear, unambiguously scored, and were between 250 and $3000 \mathrm{bp}$. Examples of polymorphic bands generated by different primers are shown in Figure 2.5. 
Genetic diversity and structure in all populations - Among the twenty six populations, mean $P, H$, and $I$ values were $39.33 \%, 0.1359$, and 0.2016 respectively. Among wild populations, mean $P, H$, and $I$ values were $37.59 \%, 0.1253$, and 0.1883 respectively. Within wild populations, lowest genetic variability was in population WV-W_21 $(P=$ $15.48 \%, H=0.0570, I=0.0655)$ whereas population PA-W_2 had the highest genetic variability $(P=76.19 \%, H=0.2060, I=0.3226)$. Among cultivated populations, mean $P, H$, and $I$ values were $41.36 \%, 0.1482$, and 0.2171 respectively. Within cultivated populations, lowest genetic variability was in populations WV-C_6 $(P=16.67 \%, H=$ $0.0470, I=0.0748)$ whereas population WV-C_8 had the highest genetic variability $(P=$ 64.29\%, $H=0.2941, I=0.3691)$ (Table 2.3).

Overall cultivated populations had higher genetic variation than wild populations evaluated in this study; however Mann-Whitney test revealed no significant differences in any genetic diversity indices. The fixation index $\Phi_{\mathrm{ST}}$ analogous to $\mathrm{F}_{\mathrm{ST}}$ (Wright, 1951) obtained by AMOVA using pairwise distances among populations was equal to 0.473 (range $0.082-0.678$ ). Which means that $47.3 \%$ of total molecular variance was attributed to among population differentiation, and $52.7 \%$ of the variance attributed to individuals within populations and all values were different from zero $(P<0.001)$

\section{Genetic diversity within and among groups (WV vs. non-WV populations) - Overall} non-WV group had higher mean genetic variability $(P=62.85 \%, H=0.1866, I=0.2870)$ than WV group $(P=35.05 \%, H=0.1267, I=0.1861)$ (Table 2.4). The Mann-Whitney 
test revealed significant differences $(P<0.05)$ in all genetic diversity indices between the two groups (Table 2.5, Analysis 1).

The coefficient of genetic differentiation $\left(G_{S T}\right)$ for the WV group was 0.462 , meaning $46.2 \%$ of total variation was attributed to among population differentiation, and $53.8 \%$ of variation was within populations. For the non-WV group $G_{S T}$ was 0.170 , which means $17.0 \%$ of total variation was due to among population differentiation, and $83.0 \%$ of genetic variation was due to individuals within population (Table 2.4). AMOVA further revealed significant $(P<0.001)$ differentiation within groups (Table 2.6). For the WV group, among population differentiation accounted for $48.48 \%$ of the total variation and the rest $(51.52 \%)$ was attributed to individual differentiations within populations (Table 2.6, Analysis 1). For the non-WV group, $25.33 \%$ of the total variation was attributed to among population differentiation, and $74.67 \%$ was due to individual differentiation within populations (Table 2.6, Analysis 2). When total population differentiation was partitioned to a three level hierarchy to account for among groups (WV and non-WV) differentiation, highest genetic differentiation was attributed to individuals within populations $(46.50 \%)$, among population differentiation accounted for $36.84 \%$, and variation between groups accounted for $16.66 \%$ of the total variation (Table 2.6, Analysis 3).

UPGMA dendrogram based on Nei's (1978) unbiased genetic distances between WV and non-WV populations revealed two well defined clusters separating WV populations from non WV populations. Non-WV group formed two distinct sub clusters, 
one having Pennsylvania (PA) populations and the other having Wisconsin (WI) populations together. These clusters were supported by high bootstrap values suggesting a strong genetic differentiation between groups (Figure 2.6).

\section{Genetic diversity within and among ecotypes (wild vs. cultivated populations) in} WV - In cultivated ecotype, lowest mean genetic variations were in population WV-C_6 $(P=16.67 \%, H=0.0470, I=0.0748)$ whereas population WV-C_8 had the highest genetic variability $(P=64.29 \%, H=0.2941, I=0.3691)$. For wild ecotype, lowest genetic variability was in population WV-W_21 $(P=15.48 \%, H=0.0570, I=0.0655)$ and population WV-W_17 had the highest genetic variability $(P=48.81 \%, H=0.1675, I$ $=0.2506)($ Table 2.3). Overall, $\mathrm{WV}$ cultivated ecotype exhibited higher mean genetic diversity values $(P=39.13 \%, H=0.1441, I=0.2095)$ than wild ecotypes $(P=31.65 \%, \mathrm{H}$ $=0.1121, I=0.1666)($ Table 2.4). However Mann-Whitney test did not reveal significant differences in any of the diversity indices between ecotypes (Table 2.5, Analysis 2).

The coefficients of genetic differentiation $\left(G_{S T}\right)$ were 0.425 and 0.475 for cultivated and wild ecotypes respectively, meaning that $42.5 \%$ of total genetic variation in WV cultivated ecotype resides among population and 57.5 within population, whereas for wild ecotype, $47.5 \%$ of total variation was among populations and $52.5 \%$ among individual within populations (Table 2.4). AMOVA further revealed significant $(P<$ 0.001) molecular variations within ecotypes, with cultivated populations having lower among population differentiation than wild populations (Table 2.6, Analyses $4 \& 5$ ). However, when variance was partitioned to account for between ecotype variation, a 
significant $(P<0.001)$ proportion was attributable to among population differentiation $(49.10 \%)$ and to individual differentiation within populations $(51.72 \%)$ but not among ecotypes $(P=0.07048)$. Variation among ecotypes was very small (close to zero), so a negative value was obtained (Table 2.6, Analysis 6).

UPGMA cluster analysis based on Nei's (1978) unbiased genetic distances among $22 \mathrm{WV}$ populations did not separate populations into clusters consistent with their ecotypes. Populations from both ecotypes clustered with each other indicating that they were not distinctly separable from each other genetically, and clusters were supported by low bootstrap values (Figure 2.7).

\section{Genetic diversity within and among populations from low (WVCR1) and high} (WVCR2) cultivation intensity regions in WV - In WVCR1 populations, $P$ values ranged from $16.67 \%$ to $36.90 \%, H$ ranged from 0.0470 to 0.1589 , and $I$ ranged from 0.0748 to 0.2275 for WV-C_6 and WV-C_1 populations respectively (Table 2.3). Whereas in WVCR2 populations, $P$ ranged from $53.71 \%$ to $64.29 \%, H$ ranged from 0.2329 to 0.2941 , and $I$ ranged from 0.2960 to 0.3691 for WV-C_10 and WV-C_8 populations respectively (Table 2.3). Mean estimates of genetic diversity of populations in WVCR2 were higher $(P=60.60 \%, H=0.2593, I=0.3243)$ than that of populations in WVCR1 $(P=29.93 \%, H=0.0948, I=0.1603)$ (Table 2.4). Mann-Whitney test indicated significant differences $(P<0.05)$ in all genetic variation measures between populations from low and high cultivation intensity regions (Table 2.5, Analysis 3). 
The estimates of genetic differentiation $\left(G_{S T}\right)$ were 0.4140 and 0.3410 for WVCR1 and WVCR2 populations respectively, meaning $44.10 \%$ of total variation was among populations and $55.90 \%$ within populations in WVCR1, whereas $34.10 \%$ of total variation was among populations and $64.10 \%$ within populations in WVCR2 (Table 2.4). When genetic differentiation was partitioned to account for among regions variation using AMOVA, it further revealed higher proportion of differentiation (53.68\%) attributed to individuals in populations within regions, followed by among populations within regions $(42.11 \%)$ and differentiation among regions was the lowest $(4.20 \%)$ but was highly significant (Table 2.6, Analysis 7).

\section{Genetic diversity within and among populations from low and high harvest pressure} regions in WV - In WVWR1 populations, $P$ ranged from $20.24 \%$ to $48.81 \%, H$ ranged from 0.0687 to 0.1675 , and $I$ ranged from 0.1032 to 0.2506 for WV-W_14 and WVW_17 populations respectively (Table 2.3 ). Whereas for populations within WVWR2, $P$ ranged from $15.48 \%$ to $38.10 \%, H$ ranged from 0.0570 to 0.1193 , and $I$ ranged from 0.0655 to 0.1820 for WV-W_21 and WV-W_22 populations, respectively (Table 2.3). Mean estimates of genetic variation within populations in WVWR1 region were higher $(P=33.33 \%, H=0.1172$, and $I=0.1743)$ compared to populations in WVWR2 region

$(P=28.27 \%, H=0.1019$, and $I=0.1513)($ Table 2.4). However Mann-Whitney test did not indicated significant differences in all variation measures between regions (Table 2.5, Analysis 4). 
The coefficients of genetic differentiation $\left(G_{S T}\right)$ were 0.454 and 0.459 for WVWR1 and WVWR2 populations respectively, inferring an almost equal proportion of among populations variation for populations in both regions (Table 2.4). A three level hierarchy AMOVA revealed significant $(P<0.001)$ genetic differentiation among populations within regions and individuals in populations within regions, however variation between regions was not significant $(P=0.79179)$ and resulted in a negative value (Table 2.6, Analysis 8).

\section{Comparisons between all WV wild populations and populations from WVCR1 or} WVCR2 - Comparisons between 12 wild populations and 7 cultivated populations in low cultivation intensity regions (WVCR1) revealed non-significant differences in all genetic diversity measures (Table 2.5, Analysis 5). However comparisons between the same 12 wild populations and 3 cultivated populations from high cultivation intensity region (WVCR2) revealed significant differences among all genetic diversity measures (Table 2.5, Analysis 6).

UPGMA cluster analysis of WV populations in different regions based on Nei’s (1978) unbiased genetic distances among regions sub clustered WVCR1 populations close to populations from wild ecotypes (WVWR1 and WVWR2), whereas populations from WVCR2 sub clustered separately from all three regions populations (Figure 2.8). 


\section{Discussion}

Genetic diversity and structure of $\boldsymbol{P}$. quinquefolius - Using RAPD markers, this study evaluated genetic variation and structure among 26 populations of $P$. quinquefolius. Mean values of genetic diversity showed that cultivated populations had higher levels of diversity compared to wild populations. This observation is similar to that reported by Grubbs and Case (2004) for 44 populations of $P$. quinquefolius sampled from different regions across ginseng's native range. On average genetic diversity values obtained in this study were higher than those reported for 21 populations (Cruse-Sanders and Hamrick 2004) and 44 populations (Grubbs and Case 2004) of P. quinquefolius using allozymes. This can be attributed to the vast numbers of loci that can be examined with RAPD markers compared to allozymes, therefore able to reveal a high degree of DNAlevel variation. Allozymes are only limited to protein coding regions and may not be representative of genome wide diversity (Stewart and Excoffier 1996). It is also possible that differences in these values could be due to sampling from different populations, even though this study and the other two studies sampled plants from the same geographic area in WV. Genetic differentiations among and within populations were highly significant. Mean fixation index $\Phi_{\text {ST }}$ value was 0.473 . According to Wright (1978), $F_{\text {ST }}$ values above 0.25 indicate substantial genetic differentiation. This observation is consistent to what was reported for 18 wild populations of $P$. quinquefolius $\left(\mathrm{F}_{\mathrm{ST}}=0.547\right)$ in a previous study (Mooney 2007).

\section{Comparison of genetic diversity between WV and non WV populations - Means of} genetic diversity indices were lower for WV populations $(P=35.05, H=0.1267, I=$ 
$0.1861)$ compared to non West Virginia populations $(P=62.85, H=0.1866, I=0.2866)$.

Lower genetic diversity in WV populations can be attributed to harvest pressure associated with the long history of wild ginseng harvesting in WV. American ginseng populations facing harvest pressure have been reported to have reduced genetic variations compared to populations in protected areas (low pressure) (Cruse-Sanders and Hamrick 2004). Indeed when wild populations in WV were compared directly to wild populations form PA which have not been harvested for several years (protected), measures of genetic diversity in WV wild populations were almost half that of PA wild populations. The partitioning of genetic structure of WV populations was almost equal for among and within populations differentiation, however for non WV populations within population differentiation was almost three times that of among population differentiation. An almost equal genetic differentiation among and within populations from WV can be explained by lack of significant genetic differences among wild and cultivated populations from low cultivation intensity region.

\section{Genetic diversity and structure of population ecotypes in WV - There were no} significant differences in all diversity measures among cultivated and wild populations in WV. Lack of significant differences in genetic diversity among ecotypes was mainly attributed to high level of similarity between wild populations and cultivated populations from low cultivation intensity regions (WVCR1) which could be attributed to growers in these regions recruiting into their farms seeds collected from wild populations. The Majority of growers from low cultivation intensity regions (WVCR1) were also wild ginseng diggers, as most of them did help in locating wild ginseng populations used in 
this study. Although it is highly recommended that ginseng diggers plant seeds at locations from where they collect wild roots in order to sustain future ginseng populations, it is not uncommon for diggers to take along with them some seeds to start ginseng crops in their own property. After all, these seeds do not have associated costs (as opposed to expensive commercial seeds) and establishing ginseng populations on their personal property ensures easy accessibility and security of their crop from other diggers.

Most of the variation in cultivated ecotypes was attributed to individuals within populations, whereas in wild ecotypes greater differentiation was partitioned to differentiation among populations (AMOVA). This finding is in agreement with other studies on P. quinquefolius (Schluter and Punja 2002; Mooney 2007). Cultivated populations typically have a high degree of gene flow among population mainly due to movement of seeds between growers as opposed to wild populations which have mostly become isolated because of fragmented habitats therefore there is limited gene flow among populations.

Effects of cultivation intensity on genetic diversity - Higher diversity indices were observed in populations from high cultivation intensity region than from populations from low cultivation intensity regions. This can be explained by the higher gene flow in high cultivation intensity regions as a result of growers recruiting seeds from a broader source to meet their cultivation scale as opposed to lower gene flow in lower cultivation intensity regions which could be attributable to growers obtaining seeds from a narrow 
source or even collecting from a single source in the wild to start their ginseng crop. A higher proportion of among populations differentiation was present in populations from low cultivation intensity regions $\left(\mathrm{G}_{\mathrm{ST}}=0.4140\right)$ compared to among populations from high cultivation intensity regions $\left(\mathrm{G}_{\mathrm{ST}}=0.3410\right)$. This observation further supports the increased gene flow among populations in high cultivation intensity region, which increases diversity within populations but reduced genetic differentiation among populations.

\section{Genetic diversity of populations under different levels of harvest pressure in WV -}

Higher mean genetic diversity measures were observed in populations from low harvest pressure region (WVWR1) than in populations from a high harvest pressure region (WVWR2). This trend is in agreement with both field and simulation studies of $P$. quinquefolius (Cruse-Sanders and Hamrick 2004; Cruse-Sanders et al. 2005). However the differences in genetic diversity measured in this study were not significant following Mann-Whitney test and AMOVA. Lack of significant differences in genetic diversity among these regions could be attributed to small sample size evaluated from high harvest pressure regions (only 4 populations, 69 samples). Genetic structure of populations from both regions partitioned higher differentiation to individuals within populations than among populations, which again is in agreement with other studies on wild populations of American ginseng (Cruse-Sanders and Hamrick 2004; Mooney 2007)

Conservation implications - Genetic variation is a valuable resource from which populations derive short-term adaptation to environmental stochasticities and long-term 
evolutionary changes (Ellstrand and Elam 1993). This study indicates that there is still a substantial amount of genetic diversity at the population level for $P$. quinquefolius growing in WV. Harvest pressure is a factor behind diminishing genetic diversity in wild populations in WV and if left unchecked it could have far reaching consequences such as severely inbred wild populations in the long run. However if good stewardship practices for wild populations are strictly adhered to by ginseng diggers, the pace of getting to critical levels of lack of genetic diversity on wild populations can be slowed. Lack of significant differences in genetic diversity between wild and cultivated ginseng from small farms in low cultivation intensity regions imply that there is considerable movement of seeds from wild to cultivation and this may eventually lead to non distinguishable wild and cultivated genotypes in WV. 


\section{Literature Cited}

Abbo, S., J. Berger, and N.C. Turner. 2003. Evolution of cultivated chickpea: four bottlenecks limit diversity and constrain adaptation. Functional Plant Biology 30: $108-1087$.

Ahmad, B. 1998. Plant exploration and documentation in view of land clearing in Sabah. In Nair, M.N.B. and N. Ganapathi, eds., Medicinal Plants. Cure for the 21st Century. Biodiversity Conservation and Utilization of Medicinal Plants pp. 161162. Serdang, Malaysia.

Anderson, R. C., J. E. Armstrong, P. K. Benjamin, and J. S. Fralish. 1993. The ecology and biology of Panax quinquefolium L. (Araliaceae) in Illinois. The American Midland Naturalist 129: 357-372.

Anonymous, 2008. American Ginseng and the Idea of the Commons. In Tending the Commons: Folklife and Landscape in Southern West Virginia. The Library of the Congress. http://memory.loc.gov/ammem/collections/tending/essay1a.html. Accessed on April 30, 2008.

Bai, D., J. Brandle, and R. Reeleder. 1997. Genetic diversity in North American ginseng (Panax quinquefolius L.) grown in Ontario detected by RAPD analysis. Genome 40: $11-115$.

Boehm, C. L., H. C. Harrison, G. Jung, and J. Nienhuis. 1999. Organization of American and Asian ginseng germplasm using randomly amplified polymorphic DNA (RAPD) markers. Journal American Society Horticulture Science 124: 252-256. 
Canter, P.H. 2005. Bringing medicinal plants into cultivation. Focus on Alternative and Complementary Therapy 10: 167-168.

Carpenter, S. G., and G. Cottam. 1982. Growth and reproduction of American ginseng (Panax quinquefolius) in Wisconsin, USA. Canadian Journal of Botany 60: 2692-2696.

Catling, P. M., E. Haber, and E. Small. 1994. Poorly known economic plants of Canada: ginseng_Panax quinquefolius L. Canadian Botanical Association Bulletin 27: $70-72$.

Charron, D., and D. Gagnon. 1991. The demography of northern populations of Panax quinquefolium (American ginseng). Journal of Ecology 79: 431-445.

Cheng, L. and P.D. Mitchell. 2009. Status of the Wisconsin Ginseng Industry. Department of Agricultural and Applied Economics, University of Wisconsin-Madison, Madison, Wisconsin, USA.

Christensen, L.P., M. Jensen, and U. Kidmose. 2006. Simultaneous determination of ginsenosides and polyacetylenes in American ginseng root (Panax quinquefolium L.) by high performance liquid chromatography. Journal of Agricultural and Food Chemistry 54: 8995-9003.

Cruse-Sanders, J.M., and J.L. Hamrick. 2004. Genetic diversity in harvested and protected populations of wild American ginseng, Panax quinquefolius L. (Araliaceae). American Journal of Botany 91 (4): 540-548.

Cruse-Sanders, J.M., J.L. Hamrick, and J.A. Ahumada. 2005. Consequences of harvesting for genetic diversity in American ginseng (Panax quinquefolius L.): a simulation study. Biodiversity and Conservation 14: 493-504. 
Cunningham, A.B. 1993. African medicinal plants. Setting priorities at the interface between conservation and primary healthcare. People and Plants Working Paper 1. UNESCO, Paris, France.

Doebley, J. 1989. Isozymic evidence and evolution of crop plants. In: Soltis E.D. and Soltis P.M. (eds), Isozymes in Plant Biology. Dioscorides, Portland, Oregon, pp $165-191$.

Ellstrand, N.C., and D.R. Elam. 1993. Population genetic consequences of small population size: implications for plant conservation. Annual Review for Ecology and Systematics 24: 271-242.

Excoffier, L., P.E. Smouse, and J.M Quatro.1992. Analysis of molecular variance inferred from metric distances among DNA haplotypes: application to human mitochondria DNA restriction data. Genetics 131 (2):479-491.

Excoffier, L., G. Laval, and S. Schneider. 2005. Arlequin ver. 3.11: An integrated software package for population genetics data analysis. Evolutionary Bioinformatics Online 1:47-50.

Giblette, J. 2006. The Role of Cultivation in Conserving Medicinal Plants. In Call et al., 2006. Mending the Web of Life: Chinese Medicine and Species Conservation. Published by the International Fund for Animal Welfare (IFAW) and the Foundation for Education and Research on Botanicals, American Herbal Products Association (AHPA-ERB).

Grubbs, J.H., and M.A. Case. 2004. Allozyme variation in American ginseng (Panax quinquefolius L.): Variation, breeding system, and implications for current conservation practice. Conservation genetics 5: 13-23. 
Hamilton, A.C. 1997. Threats to plants: an analysis of Centres of Plant Diversity. In Conservation into the 21 st Century. Proceedings of $4^{\text {th }}$ International Botanic Gardens Conservation Congress (ed. D. H. Touchell and K. W. Dixon), pp. 309322. Kings Park and Botanic Garden, Perth, Australia.

Hernandez-Verdugo, S., R. Luna-Reyes, and K. Oyama. 2001. Genetic structure and differentiation of wild and domesticated populations of Capsicum annuum from Mexico. Plant Systematics and Evolution 226: 129-142.

Hu, Y. S., L. Rudenberg, and P. D. Tredici. 1980. Studies of American Ginseng. Rhodora 82: 627-638.

Kuipers, S.E. 1997. Trade in medicinal plants. In Medicinal plants for forest conservation and healthcare, vol. 11. Non-Wood Forest Products (ed. G. Bodeker, K. K. S. Bhat, J. Burley and P. Vantomme), pp. 45-59. FAO, Rome, Italy.

Lange, D. 1998. Europe's medicinal and aromatic plants: their use, trade and conservation: an overview. TRAFFIC International, Cambridge, UK.

Lewis, W. H. 1988. Re-growth of a Decimated Population of (Panax quinquefolium) in a Missouri Climax Forest. Rhodora. 90: 1-5.

Lewis, W., and V. Zenger. 1982. Population dynamics of American ginseng (Panax quinquefolium) Araliaceae. American Journal of Botany. 69 (9): 1483-1490.

Lewis, W., and V. Zenger. 1983. Breeding systems and fecundity in the American ginseng, Panax quinquefolium (Araliaceae). American Journal of Botany 70 (3): 466-468. 
Lewontin, R.C. 1972. The apportionment of human diversity. Evolutionary Biology 6: $381-398$

Lim, W. 2004. Population characterization of Panax quinquefolium with ginsenoside analysis and randomly amplified polymorphic DNA markers. Ph.D. dissertation. Cornell University, Ithaca, NewYork.USA.

Miller, M.P. 1997. AMOVA-PREP: A program for the preparation of input files for use in WINAMOVA. Department of Biological Sciences, Northern Arizona University, Flagstaff, Arizona.USA.

Miller, M.P. 1998. TFPGA: A Windows ${ }^{\circledR}$ program for the analysis of allozyme and molecular population genetic data. Department of Biological Sciences, Northern Arizona University, Flagstaff, Arizona.

Miller, J.A., and B.A. Schaal. 2006. Domestication and the distribution of genetic variation in wild and cultivated populations of the Mesoamerican fruit tree Spondias purpurea L. (Anacardiaceae). Molecular Ecology. 15: 1467-1480.

Mooney, H.E. 2007. Genetic and evolutionary consequences of harvest in American ginseng, Panax quinquefolius L. (Araliaceae). Ph.D. dissertation. West Virginia University, Morgantown, West Virginia.USA.

Nei, M. 1973. Analysis of gene diversity in subdivided populations. Proceedings of the National Academy of Science 70: 3321-3323.

Nei, M. 1978. Estimation of average heterozygosity and genetic distance from a small number of individuals. Genetics 89: 583-590.

O’Hara, M., D. Kiefer, K. Farrell, and K. Kemper. 1998. A review of 12 commonly used medicinal herbs. Archives of Family Medicine 7: 523-53. 
Otero-Arnaiz A., A. Casas, J.L. Hamrick, and J. Cruse-Sanders. 2005. Genetic variation and evolution of Polaskia chichipe (Cactaceae) under domestication in the Tehuacán Valley, central Mexico. Molecular Ecology 14: 1603-1611.

Pritts, K. D. 1995. How to find, grow, and use America's forest gold. Stackpole Books. Mechanicsburg, Pennsylvania, USA.

Proctor J. T. A. and W. G. Bailey. 1987. Ginseng: Industry, botany, and culture. Horticulture Reviews 9: 187-236.

Schlessman, M. 1985. Floral biology of American ginseng (Panax quinquefolium). Bulletin of the Torrey Botanical Club 112 (2): 129-133.

Schluter, C., and Z.K. Punja. 2002. Genetic diversity among natural and cultivated field populations and seed lots of American ginseng (Panax quinquefolius L.) in Canada. International Journal of Plant Science 163: 427-439.

Stewart Jr., C.N., and D.M. Porter. 1995. RAPD profiling in biological conservation: an application to estimating clonal variation in rare and endangered Iliamna in Virginia. Biological Conservation 74: 135-142.

Stewart Jr., C.N., and L. Excoffier. 1996. Assessing population genetic structure and variability with RADP data: Application to Viccinium macrocarpon (American Cranberry). Journal of Evolutionary Biology 9: 153-171.

Vance N.C. 2002. Ecological considerations in sustainable use of wild plants. In: Jones E.T., McLain R.J.and Weigand J. (eds) Nontimber Forest Products in the United States. pp. 151-162. University Press of Kansas, Lawrence, Kansas, USA.

Wright, S. 1951. The genetical structure of populations. Annals of Eugenics 15: 323354. 
Wright, S. 1978. Evolution and the genetics of populations, Volume 4, Variability within and among natural populations, University of Chicago Press, Chicago.USA.

WVDOF. 2008. http://www.wvforestry.com/ginseng.cfm?menucall=ginseng. Accessed April 22, 2008.

Yeh, C.F., R-C. Yang, T. Boyle., Z. Ye, and J.X. Mao. 1997. POPGENE. The userfriendly shareware for population genetic analysis. Molecular Biology and Biotechnology Centre, University of Alberta, Edmonton, Canada.

Zohary, D. 2004. Unconscious selection and the evolution of domesticated plants. Economic Botany 58 (1): 5-10. 


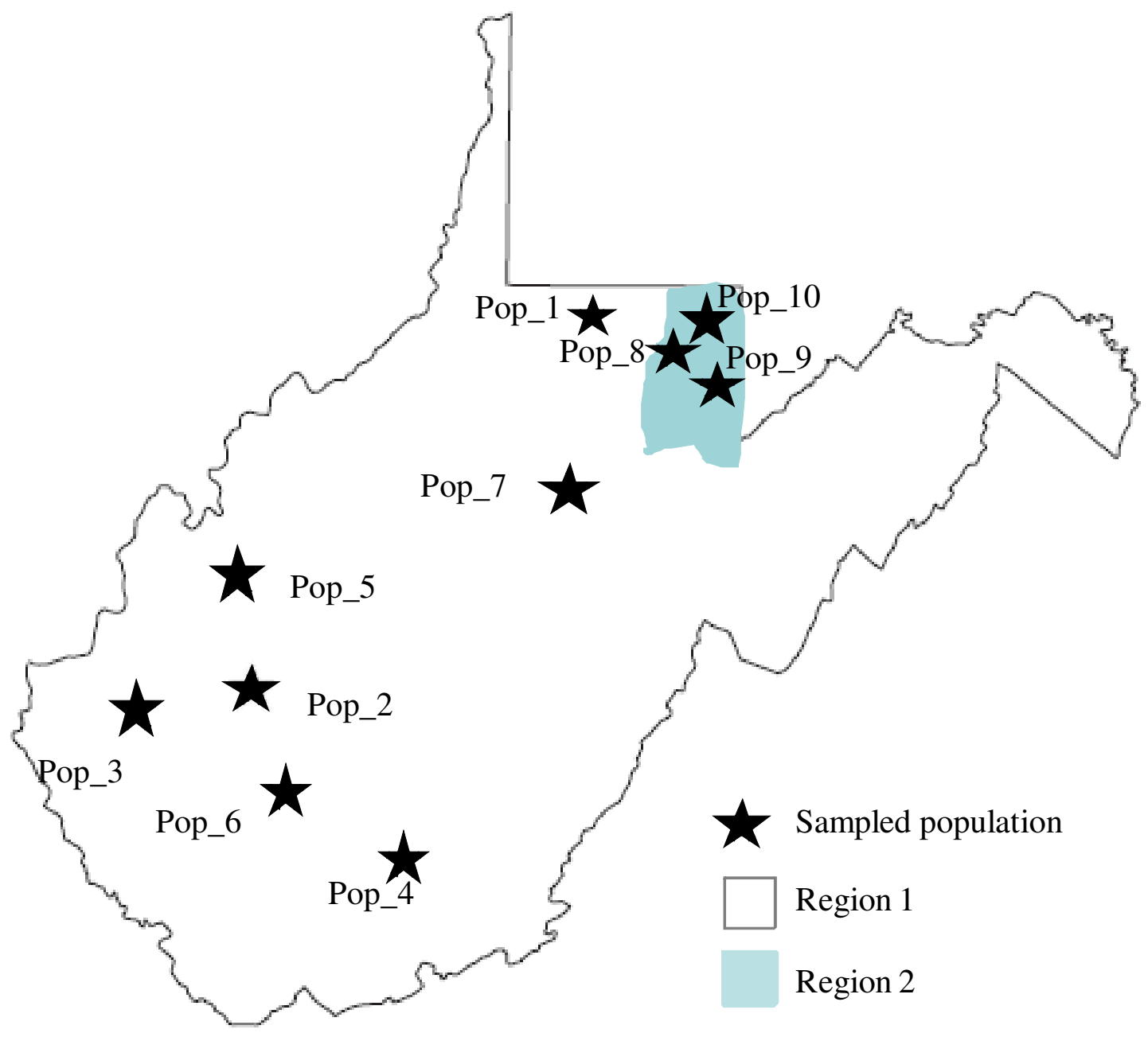

Figure 2.1. Map of West Virginia showing Panax quinquefolius cultivation regions and population sites where samples were collected. Region attributes are described in the text. Areas not drawn to scale. 


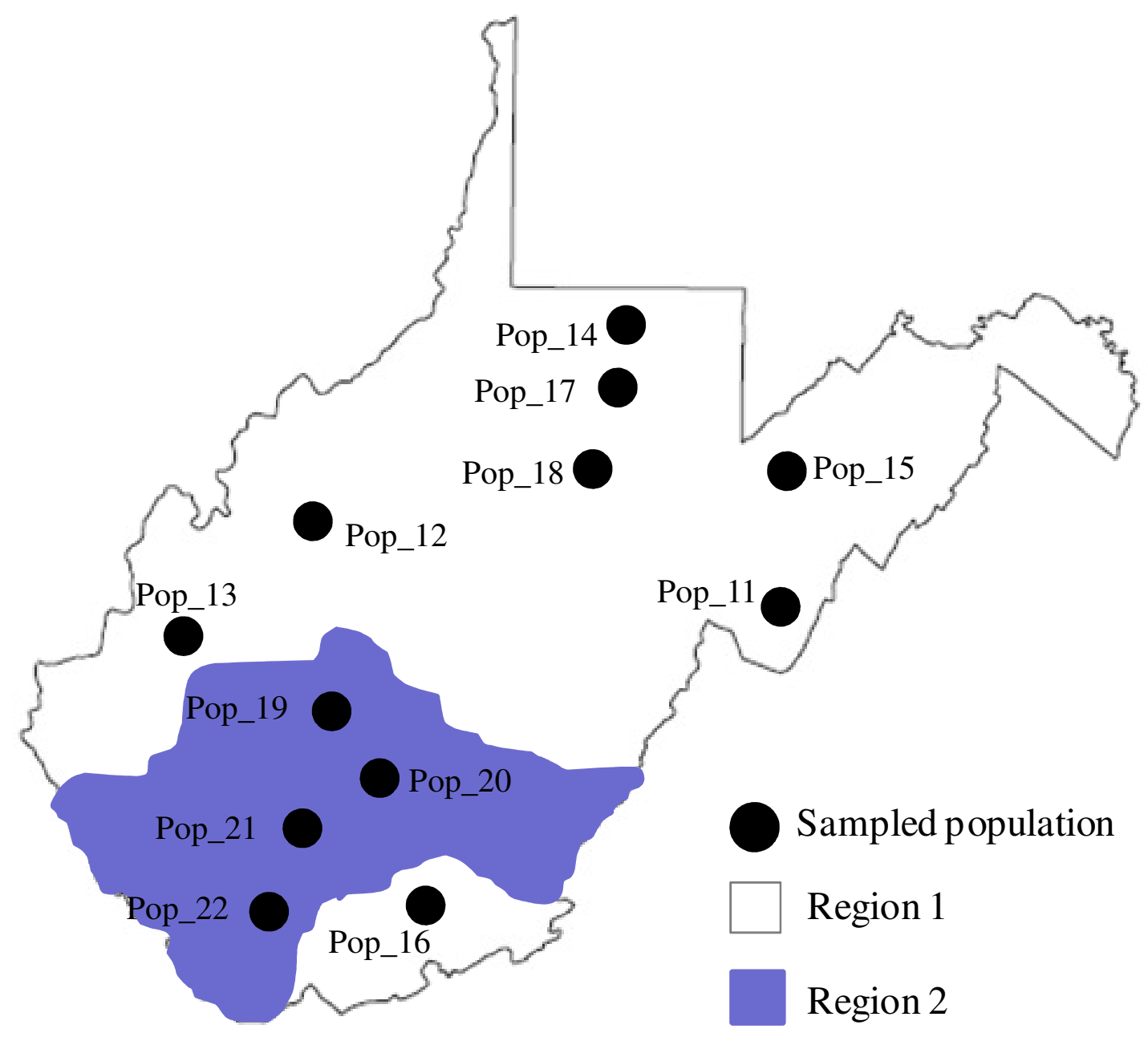

Figure 2.2. Map of West Virginia showing wild ginseng harvest regions and populations sites where samples were collected. Region attributes are described in the text. Areas not drawn to scale. 


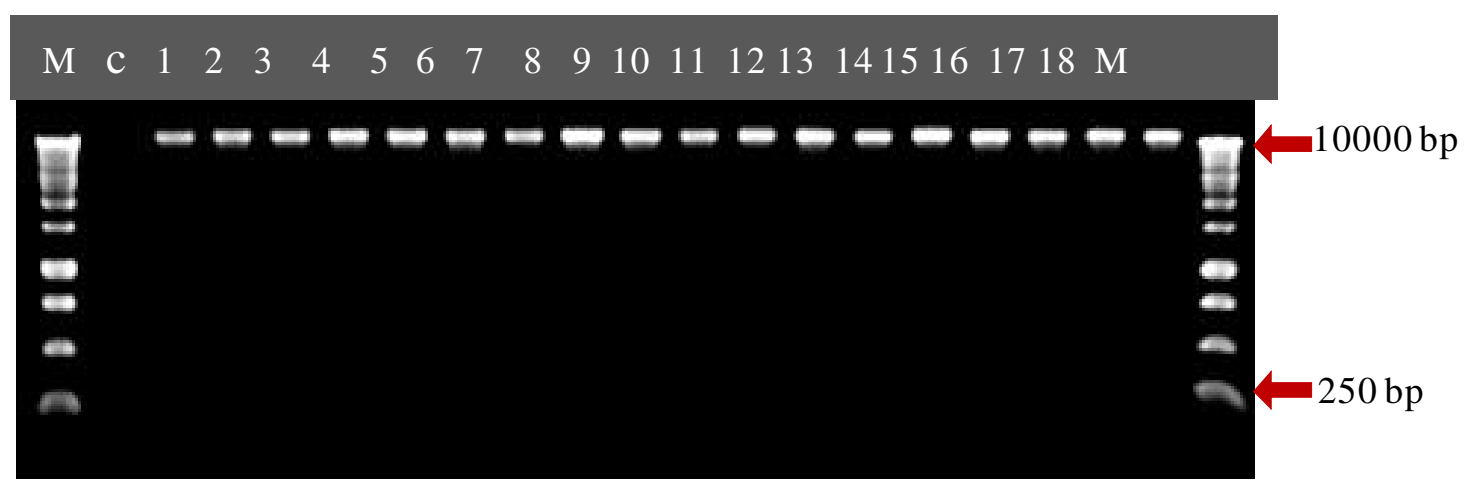

Figure 2.3. Genomic DNA isolated for Panax quinquefolius leaf samples using Mini plant genomic DNA isolation kit (Sigma-Aldrich). $1.5 \%$ agarose gel, $\mathrm{M}=1 \mathrm{~kb}$ DNA ladder, $\mathrm{C}=$ negative control, 1-18 = ginseng leaf samples. 


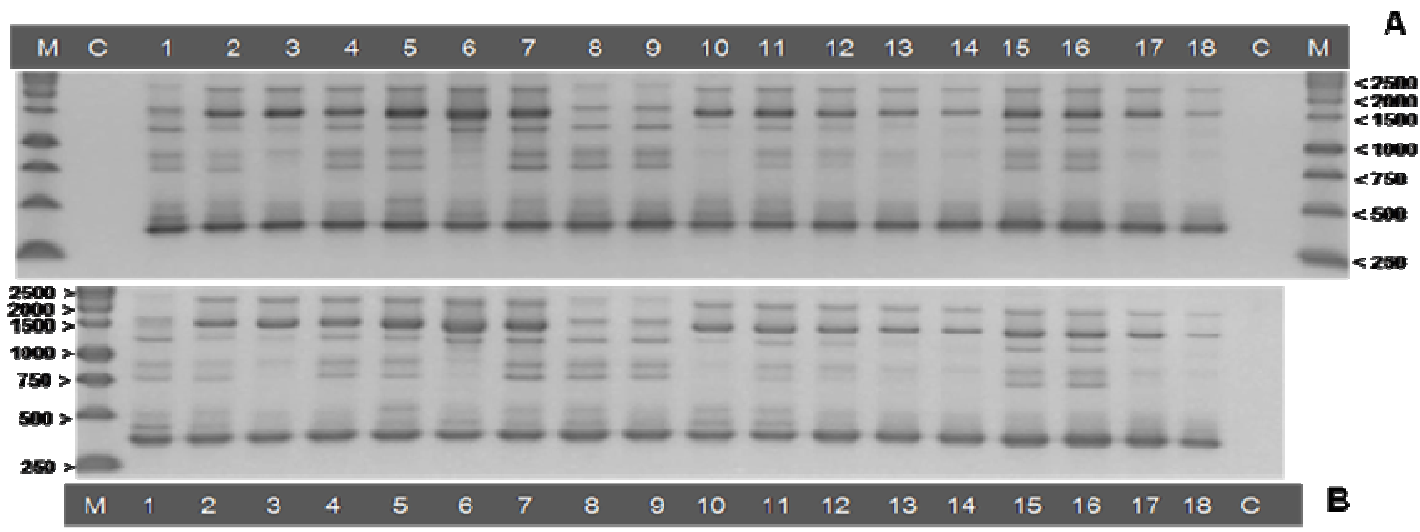

Figure 2.4. Reproducibility of band patterns A and B generated by primer UBC-98 in samples $1-18$ of POP_12. $\mathrm{M}=1 \mathrm{~kb}$ DNA marker, $\mathrm{C}=$ negative control. $\mathrm{PCR}$ reactions were run in separate days. 

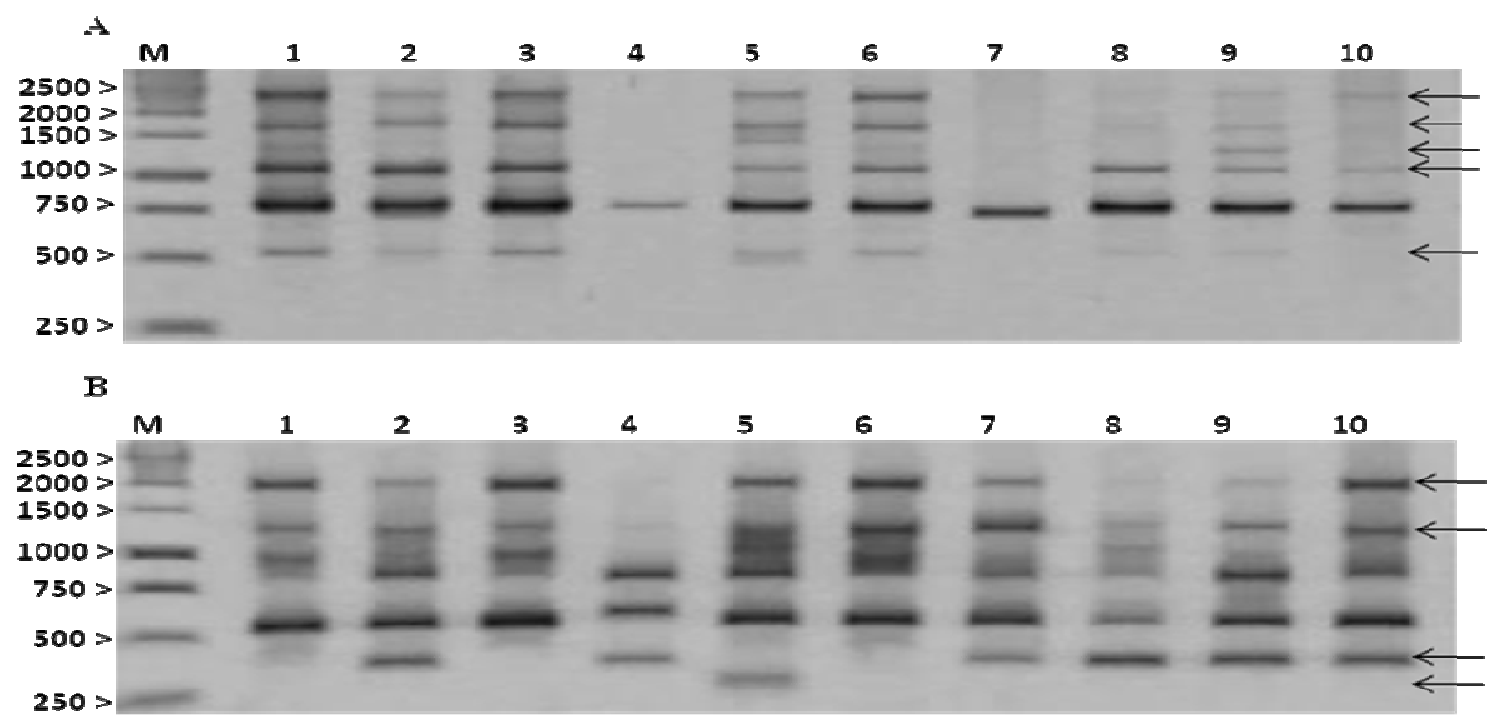

Figure 2.5. Band patterns generated by primers OPAD-11(A) and OPO-15 (B) in samples 1-10 of POP_13. $\mathrm{M}=1 \mathrm{~kb}$ DNA ladder, arrows indicate polymorphic bands. 


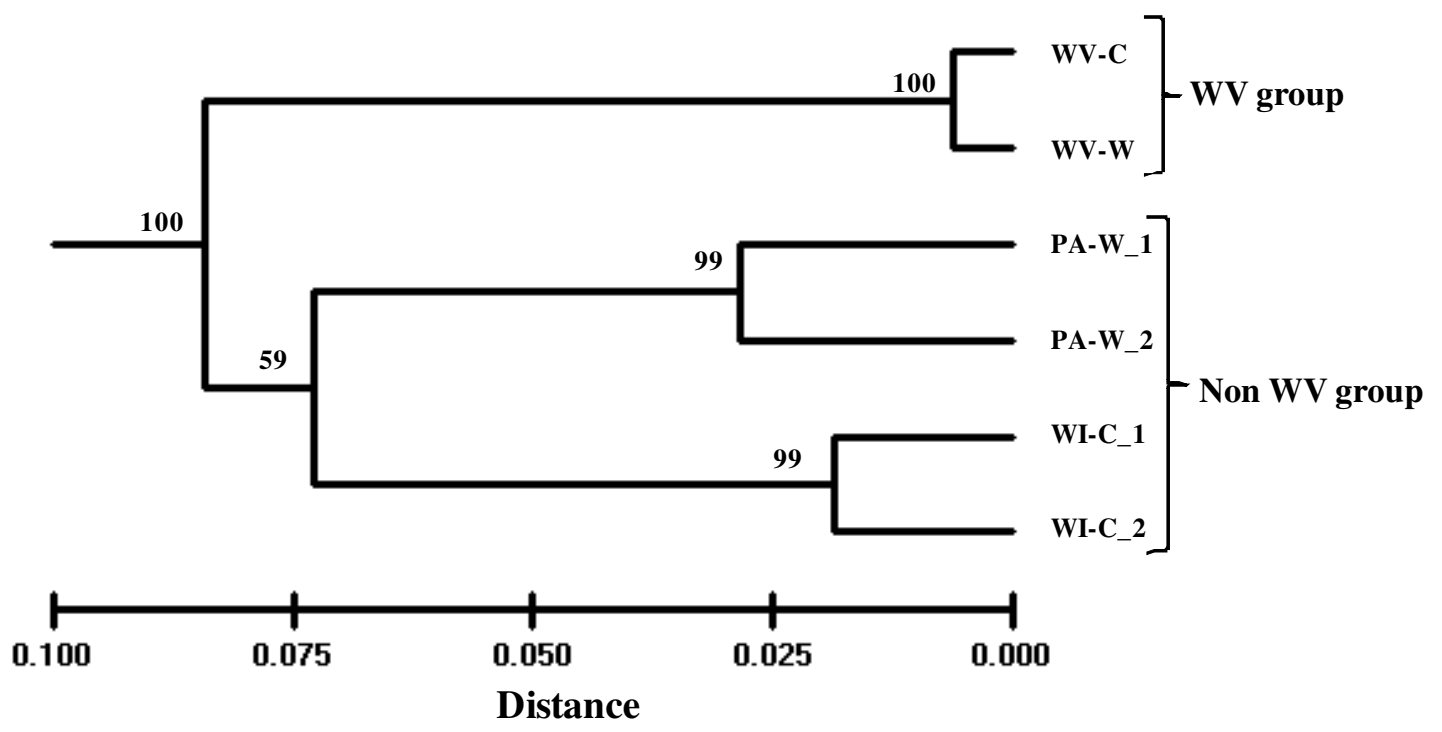

Figure 2.6. Cluster analysis of populations from groups of American ginseng using UPGMA based on Nei's (1978) unbiased genetic distance with 1000 bootstraps. Population code followed by $-\mathrm{C}$ indicates cultivated population, and $-\mathrm{W}$ indicates wild population. 


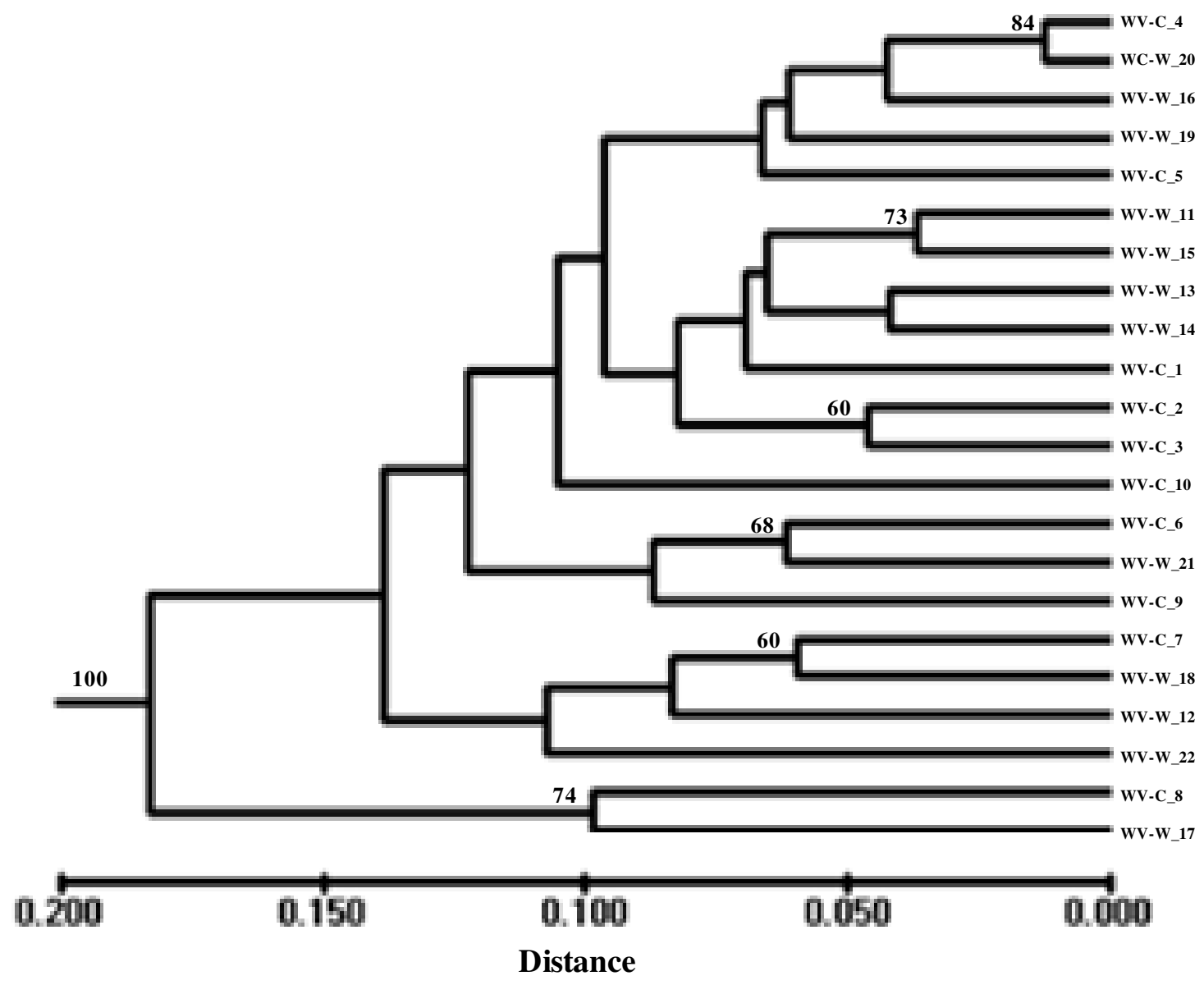

Figure 2.7. Cluster analysis of West Virginia populations using UPGMA based on Nei's (1978) unbiased genetic distances with 1000 bootstraps. Population code followed by -C indicates cultivated population, and -W indicates wild population. Only bootstrap values greater than $60 \%$ are shown. 


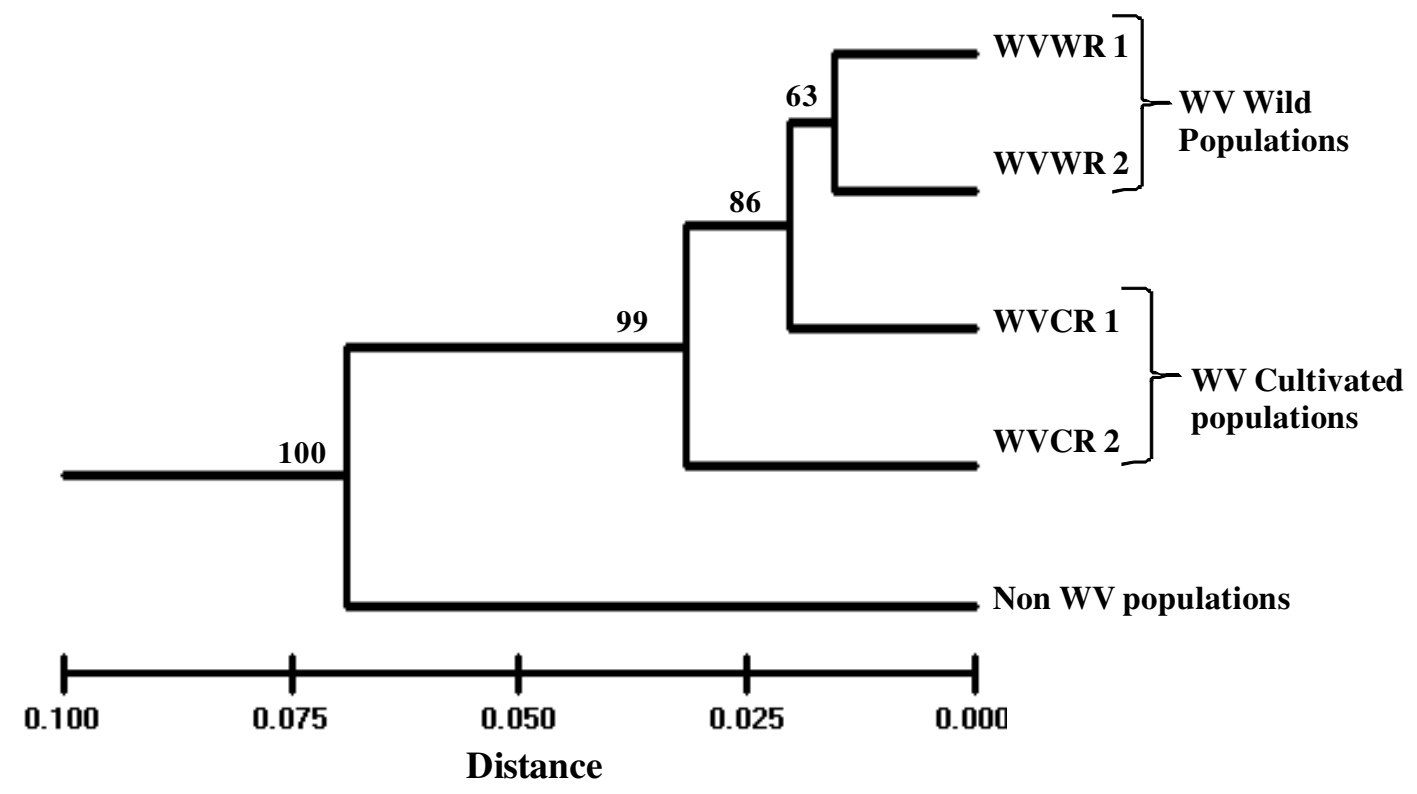

Figure 2.8. Cluster analysis of American ginseng populations from different groups, ecotypes and regions using UPGMA based on Nei's (1978) unbiased genetic distance with 1000 bootstraps. 
Table 2.1. Population, ecotype, sample size, and population code of populations used in this study.

\begin{tabular}{|c|c|c|c|}
\hline Population & Ecotype & $\begin{array}{c}\text { Sample } \\
\text { size }\end{array}$ & $\begin{array}{l}\text { Population } \\
\text { Code }\end{array}$ \\
\hline \multicolumn{4}{|l|}{ WV populations } \\
\hline POP_1 & Cultivated & 16 & WV-C_1 \\
\hline POP_2 & " & 16 & WC-C_2 \\
\hline POP_3 & & 20 & WC-C_3 \\
\hline POP_4 & $"$ & 10 & WC-C_4 \\
\hline POP_5 & $"$ & 10 & WC-C_5 \\
\hline POP_6 & $"$ & 15 & WC-C_6 \\
\hline POP_7 & $"$ & 25 & WC-C_7 \\
\hline POP_8 & $"$ & 20 & WC-C_8 \\
\hline POP_9 & $"$ & 25 & WC-C_9 \\
\hline POP_10 & $"$ & 40 & WC-C_10 \\
\hline POP_11 & Wild & 14 & WV-W_11 \\
\hline POP_12 & " & 18 & WV-W_12 \\
\hline POP_13 & $"$ & 10 & WV-W_13 \\
\hline POP_14 & $"$ & 16 & WV-W_14 \\
\hline POP_15 & $"$ & 15 & WV-W_15 \\
\hline POP_16 & $"$ & 18 & WV-W_16 \\
\hline POP_17 & $"$ & 20 & WV-W_17 \\
\hline POP_18 & $"$ & 12 & WV-W_18 \\
\hline POP_19 & $"$ & 9 & WV-W_19 \\
\hline POP_20 & $"$ & 10 & WV-W_20 \\
\hline POP_21 & $"$ & 25 & WV-W_21 \\
\hline POP_22 & $"$ & 25 & WV-W_22 \\
\hline \multicolumn{4}{|c|}{ Non WV populations } \\
\hline \multirow[t]{2}{*}{ Pennsylvania } & Wild & 16 & PA-W_1 \\
\hline & Wild & 20 & PA-W_2 \\
\hline \multirow[t]{2}{*}{ Wisconsin } & cultivated & 21 & WI-C_1 \\
\hline & cultivated & 22 & WI-C_2 \\
\hline
\end{tabular}


Table 2.2. List of primers used in this study.

\begin{tabular}{|l|l|c|c|l|}
\hline Primer & Sequence 5' $\mathbf{- 3}^{\prime}$ & $\begin{array}{c}\text { No. of } \\
\text { bands }\end{array}$ & $\begin{array}{c}\text { No. of } \\
\text { polymorphic } \\
\text { bands }\end{array}$ & $\begin{array}{l}\text { Size ranges (bp) } \\
\text { of polymorphic } \\
\text { bands }\end{array}$ \\
\hline OPD-05 & TGAGCGGACA & 21 & 15 & $300-2000$ \\
\hline OPH-04 & GGAAGTCGCC & 10 & 9 & $250-2500$ \\
\hline OPH-05 & AGTCGTCCCC & 8 & 8 & $250-2500$ \\
\hline OPO-15 & TGGCGTCCTT & 14 & 12 & $300-2000$ \\
\hline OPAD-11 & CAATCGGGTC & 13 & 12 & $300-2500$ \\
\hline UBC-81 & GAGCACGGGG & 14 & 12 & $250-3000$ \\
\hline UBC-98 & ATCCTGCCAG & 10 & 8 & $250-2500$ \\
\hline UBC-164 & CCAAGATGCT & 8 & 8 & $250-2000$ \\
\hline Total & & 98 & 84 & \\
\hline
\end{tabular}


Table 2.3. Summary of within-population genetic diversity. $\mathrm{n}=$ sample size; $P=$ percent polymorphic loci; $H=$ gene diversity (Nei 1973); $I=$ Shannon's index (Lewontin 1972). Refer to introduction section for groups and regions definitions.

\begin{tabular}{|c|c|c|c|c|}
\hline Group, region, population & $\mathbf{n}$ & $\boldsymbol{P}$ & $\boldsymbol{H}$ & $I$ \\
\hline \multicolumn{5}{|l|}{ WV-group } \\
\hline \multicolumn{5}{|l|}{ WVCR1 } \\
\hline WV-C_1 & 16 & 36.90 & $0.1589(0.2190)$ & $0.2275(0.3088)$ \\
\hline WC-C_2 & 16 & 27.38 & $0.0829(0.1515)$ & $0.1286(0.2255)$ \\
\hline WC-C_3 & 20 & 28.57 & $0.0998(0.1702)$ & $0.1504(0.2497)$ \\
\hline WC-C_4 & 10 & 36.90 & $0.1287(0.1889)$ & $0.1925(0.2726)$ \\
\hline WC-C_5 & 10 & 28.57 & $0.1066(0.1847)$ & $0.1568(0.2647)$ \\
\hline WC-C_6 6 & 15 & 16.67 & $0.0470(0.1140)$ & $0.0748(0.1757)$ \\
\hline WC-C_7 & 25 & 34.52 & $0.1296(0.1930)$ & $0.1916(0.2778)$ \\
\hline \multicolumn{5}{|l|}{ WVCR2 } \\
\hline WC-C_8 & 20 & 64.29 & $0.2941(0.2037)$ & $0.3691(0.2906)$ \\
\hline WC-C_9 & 25 & 63.81 & $0.2508(0.1778)$ & $0.3077(0.2554)$ \\
\hline WC-C_10 & 40 & 53.71 & $0.2329(0.1963)$ & $0.2960(0.2814)$ \\
\hline \multicolumn{5}{|l|}{ WVWR1 } \\
\hline WV-W_11 & 14 & 34.52 & $0.1240(01913)$ & $0.1839(0.2739)$ \\
\hline WV-W_12 & 18 & 39.29 & $0.1270(0.1894)$ & $0.1914(0.2697)$ \\
\hline WV-W_13 & 10 & 32.14 & $0.1177(0.1886)$ & $0.1742(0.2708)$ \\
\hline WV-W_14 & 16 & 20.24 & $0.0687(0.1514)$ & $0.1032(0.2204)$ \\
\hline WV-W_15 & 15 & 35.71 & $0.1312(0.1965)$ & $0.1932(0.2812)$ \\
\hline WV-W_16 & 18 & 30.95 & $0.0923(0.1626)$ & $0.1417(0.2384)$ \\
\hline WV-W_17 & 20 & 48.81 & $0.1675(0.2001)$ & $0.2506(0.2863)$ \\
\hline WV-W_18 & 12 & 25.00 & $0.1090(0.1955)$ & $0.1560(0.2766)$ \\
\hline \multicolumn{5}{|l|}{ WVWR2 } \\
\hline WV-W_19 & 9 & 27.38 & $0.1184(0.2003)$ & $0.1697(0.2835)$ \\
\hline WV-W_20 & 10 & 32.14 & $0.1130(0.1860)$ & $0.1679(0.2665)$ \\
\hline WV-W_21 & 25 & 15.48 & $0.0570(0.1373)$ & $0.0655(0.2036)$ \\
\hline WV-W_22 & 25 & 38.10 & $0.1193(0.1781)$ & $0.1820(0.2585)$ \\
\hline \multicolumn{5}{|l|}{ Non-WV group } \\
\hline WI-C_1 & 21 & 52.58 & $0.1700(0.1924)$ & $0.2577(0.2780)$ \\
\hline WI-C_2 & 22 & 52.38 & $0.1675(0.1971)$ & $0.2525(0.2822)$ \\
\hline $\mathrm{PA}-\mathrm{W}_{-} 1$ & 16 & 70.24 & $0.2028(0.1852)$ & $0.3135(0.2614)$ \\
\hline PA-W_2 & 20 & 76.19 & $0.2060(0.1756)$ & $0.3226(0.2476)$ \\
\hline Cultivated (12 populations) & 20 & 41.36 & 0.1482 & 0.2171 \\
\hline Wild (14 populations) & 16 & 37.59 & 0.1253 & 0.1883 \\
\hline All (26 populations) & 18 & 39.33 & 0.1359 & 0.2016 \\
\hline
\end{tabular}


Table 2.4. Summary of mean genetic diversity of populations in groups, ecotypes and regions. $\mathrm{n}=$ sample size; $P$ = percent polymorphic loci; $H=$ gene diversity (Nei 1973); $I$ $=$ Shannon's index (Lewontin 1972), $G_{S T}=$ coefficient of genetic differentiation. Refer to introduction section for groups, ecotypes, and regions definitions.

\begin{tabular}{cccccc}
\hline Group, ecotype, region & $\mathbf{n}$ & $\boldsymbol{P}$ & $\boldsymbol{H}$ & $\boldsymbol{I}$ & $\boldsymbol{G}_{\boldsymbol{S T}}$ \\
\hline WV (22 populations) & 389 & 35.05 & 0.1267 & 0.1861 & 0.4620 \\
WV cultivated (10 populations) & 197 & 39.13 & 0.1441 & 0.2095 & 0.4250 \\
WVCR1 (7 populations) & 112 & 29.93 & 0.0948 & 0.1603 & 0.4140 \\
WVCR2 (3 populations) & 85 & 60.60 & 0.2593 & 0.3243 & 0.3410 \\
WV wild (12 populations) & 192 & 31.65 & 0.1121 & 0.1666 & 0.4750 \\
WVWR1 (8 populations) & 123 & 33.33 & 0.1172 & 0.1743 & 0.4540 \\
WVWR2 (4 populations) & 69 & 28.27 & 0.1019 & 0.1513 & 0.4590 \\
Non WV ( 4 populations) & 79 & 62.85 & 0.1866 & 0.2870 & 0.1700 \\
Cultivated (2 population) & 43 & 52.48 & 0.1688 & 0.2551 & 0.0552 \\
wild (2 populations) & 36 & 73.21 & 0.2044 & 0.3181 & 0.0676 \\
\hline
\end{tabular}


Table 2.5. Mann-Whitney test for differences in mean genetic diversity indices among populations of $P$. quinquefolius in various groups, ecotypes, and regions comparisons.

Analysis 1. WV vs. non-WV populations (groups)

\begin{tabular}{lcccc}
\hline Groups & $\mathrm{n}$ & $P$ & $H$ & $I$ \\
\hline WV (22 populations) & 18 & 35.05 & 0.1267 & 0.1861 \\
non WV (4 populations) & 20 & 62.85 & 0.1866 & 0.2870 \\
Significance & & $*$ & $*$ & $*$ \\
\hline
\end{tabular}

Analysis 2. WV cultivated vs. WV wild populations (ecotypes)

\begin{tabular}{lcccc}
\hline Ecotypes & $\mathrm{n}$ & $P$ & $H$ & $I$ \\
\hline Cultivated (10 populations) & 20 & 39.13 & 0.1441 & 0.2095 \\
Wild (12 populations) & 16 & 31.65 & 0.1121 & 0.1666 \\
Significance & & $\mathrm{NS}$ & $\mathrm{NS}$ & $\mathrm{NS}$ \\
\hline
\end{tabular}

Analysis 3. WV cultivated populations from low vs. high cultivation intensity regions

\begin{tabular}{lcccc}
\hline Regions & $\mathrm{n}$ & $P$ & $H$ & $I$ \\
\hline WVCR1 (7 populations) & 16 & 29.93 & 0.0948 & 0.1603 \\
WVCR2 (3 populations) & 28 & 60.60 & 0.2593 & 0.3243 \\
Significance & & $*$ & $*$ & $*$ \\
\hline
\end{tabular}

Analysis 4. WV wild populations from low vs. high harvest regions

\begin{tabular}{lcccc}
\hline Regions & $\mathrm{n}$ & $P$ & $H$ & $I$ \\
\hline WVWR1 (8 populations) & 15 & 33.33 & 0.1172 & 0.1743 \\
WVWR2 (4 populations) & 17 & 28.27 & 0.1019 & 0.1513 \\
Significance & & $\mathrm{NS}$ & $\mathrm{NS}$ & $\mathrm{NS}$ \\
\hline
\end{tabular}

Anlaysis 5. WV wild populations vs. WV cultivated populations from low cultivation intensity region

\begin{tabular}{lcccc}
\hline Population & $\mathrm{n}$ & $P$ & $H$ & $I$ \\
\hline Wild (12 populations) & 16 & 31.65 & 0.1121 & 0.1666 \\
WVCR1(7 populations) & 16 & 29.93 & 0.0948 & 0.1603 \\
Significance & & $\mathrm{NS}$ & $\mathrm{NS}$ & $\mathrm{NS}$ \\
\hline
\end{tabular}

Anlaysis 6. WV wild populations vs. WV cultivated populations from high cultivation intensity region

\begin{tabular}{lcccc}
\hline Population & $\mathrm{n}$ & $P$ & $H$ & $I$ \\
\hline Wild (12 populations) & 16 & 31.65 & 0.1121 & 0.1666 \\
WVCR2 (3 populations) & 28 & 60.60 & 0.2593 & 0.3243 \\
Significance & & $*$ & $*$ & $*$ \\
\hline
\end{tabular}

* Significant; NS = not significant at $\alpha=0.05$ 
Table 2.6. Summary of analysis of molecular variance (AMOVA) for groups, ecotypes and regions of $P$. quinquefolius using RAPD markers. Significance tests based on 1000 random permutations.

\begin{tabular}{|c|c|c|c|c|c|}
\hline $\begin{array}{l}\text { Source of } \\
\text { variation }\end{array}$ & df & SS & $\begin{array}{c}\text { Variance } \\
\text { components }\end{array}$ & $\begin{array}{l}\% \text { of total } \\
\text { variation }\end{array}$ & $P$-value \\
\hline \multicolumn{6}{|l|}{ Analysis 1- WV group } \\
\hline Among populations & 21 & 1938.855 & 4.96140 & 48.48 & $<0.001$ \\
\hline Within populations & 367 & 1935.279 & 5.27324 & 51.52 & $<0.001$ \\
\hline \multicolumn{6}{|l|}{ Analysis 2- Non WV group } \\
\hline Among populations & 3 & 209.845 & 3.09361 & 25.33 & $<0.001$ \\
\hline Within populations & 75 & 684.028 & 9.12038 & 74.67 & $<0.001$ \\
\hline \multicolumn{6}{|c|}{ Analysis 3- WV vs. non WV group } \\
\hline Between groups & 1 & 379.070 & 2.12286 & 16.66 & $<0.001$ \\
\hline Among populations & 24 & 2148.700 & 4.69400 & 36.84 & $<0.001$ \\
\hline Within populations & 442 & 619.307 & 5.92603 & 46.50 & $<0.001$ \\
\hline \multicolumn{6}{|c|}{ Analysis 4- WV cult. populations } \\
\hline Among populations & 9 & 862.597 & 4.67319 & 45.30 & $<0.001$ \\
\hline Within populations & 187 & 1055.332 & 5.64348 & 54.70 & $<0.001$ \\
\hline \multicolumn{6}{|c|}{ Analysis 5- WV wild populations } \\
\hline Among populations & 11 & 984.850 & 5.34205 & 52.22 & $<0.001$ \\
\hline Within populations & 180 & 879.947 & 4.88860 & 47.78 & $<0.001$ \\
\hline \multicolumn{6}{|c|}{ Analysis 6- WV population ecotypes } \\
\hline Between ecotypes & 1 & 91.408 & -0.08373 & -0.82 & 0.07048 \\
\hline Among populations & 20 & 1847.447 & 5.00559 & 49.10 & $<0.001$ \\
\hline Within populations & 367 & 1935.279 & 5.27324 & 51.72 & $<0.001$ \\
\hline \multicolumn{6}{|c|}{ Analysis 7- WV cultivation regions } \\
\hline Between regions & 1 & 159.563 & 0.44206 & 4.20 & $<0.001$ \\
\hline Among populations & 8 & 703.035 & 4.42724 & 42.11 & $<0.001$ \\
\hline Within populations & 187 & 1055.332 & 5.64348 & 53.68 & $<0.001$ \\
\hline \multicolumn{6}{|c|}{ Analysis 8- WV wild harvest regions } \\
\hline Between regions & 1 & 86.933 & -0.24968 & -2.47 & 0.79179 \\
\hline Among populations & 10 & 897.916 & 5.46870 & 54.10 & $<0.001$ \\
\hline Within populations & 180 & 879.947 & 4.88860 & 48.37 & $<0.001$ \\
\hline
\end{tabular}

$\mathrm{df}=$ degrees of freedom; SS $=$ sum of squares. 


\section{CHAPTER 3}

Variation of ginsenosides profiles and content among root morphotypes of American ginseng: economic implications 


\begin{abstract}
Quality perception and value of ginseng root is influenced by its morphotype. In this study genotypic influence on root morphotype has been elucidated, profiles and concentration of individual ginsenoside among three American ginseng root morphotypes (ML, BLB, and STK) are compared, and economic implications with regard to marketing and breeding of these morphotypes are assessed. The three morphotypes were observed in 3 month old plants that were grown from a mixed batch of seeds in a greenhouse using a growing medium of uniform composition and bulk density. This implies that underlying genetics could be playing a role in morphotype variation in ginseng roots and presents a potential for breeding desirable morphotypes, since currently there are no cultivars for this species. The frequency distribution of these root mophotypes in the greenhouse study was similar to that observed in field cultivated roots. On average the STK morphotype was the most abundant (63\%) followed by BLB (22\%) and ML morphotypes (15\%). Quantification of six ginsenosides (Rg1, Re, Rb1, Rc, Rb2, and Rd) in roots of marketable age ( $>4$ years) by HPLC showed significant variation in profiles and concentration of individual ginsenoside among morphotypes. Chromatographic patterns of $\operatorname{Rg} 1$ and Re showed two profiles; low $\operatorname{Rg} 1 /$ high Re, and high Rg1/low Re, although the profiles were not particular to any root morphotype. ML roots consistently exhibited low Rg1/high Re profiles whereas BLB and STK roots exhibited both profiles. Ginsenosides Rg1, Re, and Rb1 varied significantly among morphotypes, however Rb2, Rc and Rd ginsenosides did not. There was a significant difference in total ginsenosides content between ML roots $(2.19 \pm 0.07 \%, w / w)$ and BLB or STK roots $(1.86 \pm 0.07 \%$, $1.79 \pm 0.07 \%$ respectively), however BLB and STK roots were not significantly different.
\end{abstract}


Basing quality of root on total ginsenosides alone, it would be conclude that roots of ML morphotypes are of higher quality than those of BLB and STK morphotypes. Economic analysis showed that if a grower opts to sort out a mixed batch of roots to different morphotypes prior to marketing, revenue returns could be increased under current production system (no ginseng cultivars) only if sorting does not result in value reduction of the other two non-ML morphotypes. However, if sorting will result in devaluation of other two non-ML morphotypes, then even at the lowest sorting cost, this strategy will result in a net loss, simply because of the low abundance of ML morphotype in the population. Substantial economic gains are visible if successful breeding and production of high quality morphotype is attained in future. The results from this study provide breeding targets for American ginseng, and will have important implications to the grading of American ginseng roots. 


\section{Introduction}

American ginseng (Panax quinquefolius L.) is an economically important medicinal plant with earnings of over 100 million US dollars annually (Ren and Chen 1999). This perennial herb belongs to the Araliaceae family and is native to the eastern deciduous woodlands of North America (Catling et al. 1994). For over 300 years it has been harvested from the wild for export to Asia where ginseng is widely used in Traditional Chinese Medicine (TCM) (Duke 1989; Persons 1994; Robbins 1998). Ginseng use in western cultures as an herbal supplement has become common lately, mainly due to increasing use of herbal remedies in developed countries (Breevort 1998, Hoareau and DaSilva 1999).

The bulk of American ginseng roots sold from North America are cultivated, but considerable amounts of roots are still harvested from the wild (Pierce 2002). Although wild collection is prohibited in some states in the US, some states do allow wild collection but require ginseng diggers to obtain a permit from respective state regulatory agencies (Schumann and Thomas 1993, Robbins 1998). The regulation on collecting American ginseng from the wild was initiated following its listing as an appendix II species by CITES in 1975 (Robbins 1998). The purpose of this regulation is to ensure American ginseng population levels are sustained in the wild to avoid its extinction due to over-collection.

Medicinally, P. quinquefolius and Panax ginseng (Asian ginseng) are the two commonly used species of the genus Panax (Kitts et al. 2000). The two species grow in 
different continents however they occupy similar habitats (Pritts 1995). They are genetically different, have different ginsenoside profiles and exert opposite therapeutic properties (Sengupta et al. 2004). P. ginseng is purpoted to have stimulant properties and is therefore used to energize the body, whereas $P$. quinquefolius is purported to have calming properties and thus used as an adaptogen and a mild tonic to relax the body (Pritts 1995; Ligor 2005).

Ginsenosides are the main bioactive components in the genus Panax. . They are available in small quantities and are believed to be responsible for most of ginseng's pharmacological actions (Attele et al. 1999). More than 40 different ginsenosides have been identified in genus Panax (Teng et al. 2003). The basic structure of ginsenosides is similar, consisting of a gonane steroid nucleus with 17 carbon atoms arranged in four rings with a modified side chain at C-20 (Shibata et al. 1985; Radad et al. 2006). Based on their structural differences, ginsenosides can be classified into three groups: the protopanaxadiol group (e.g., Rb1, Rb2, Rb3, Rc, Rd, Rg3, Rh2, Rs1), the protopanaxatriol group (e.g., Re, Rf, $\mathrm{Rg} 1, \mathrm{Rg} 2, \mathrm{Rh} 1$ ), and the oleanic acid group (e.g., Ro) (Attele et al. 1999; Vanisree et al. 2004).

The specific mechanisms of pharmacological actions of ginsenosides are yet to be fully elucidated, however, it has been suggested that each ginsenoside may have its own specific tissue-dependent effects and may act independently or in combination with others to produce a biological effect (Ki et al. 1998; Murphy and Lee 2002). For instance ginsenosides $\mathrm{Rg} 1$ and $\mathrm{Rb} 1$ have been reported as efficient neuroprotective agents (Liao et 
al. 2002), they promote neural growth in vitro, protect neurons against ischemic injury (Zou 2002; Shen and Zhang 2003), enhance learning ability by increasing hippocampal synaptic density (Mook-Jung 2001), and prevent memory loss by increasing the proliferative ability of neural progenitor cells (Wen et al. 1996; Zhang et al. 1998; Shen and Zhang 2003). Ginsenoside Rb1 is also reported to have wound healing properties, as it mediates vascular regeneration by promoting angiogenesis via stimulation of vascular endothelial growth factor production (Kimura et al. 2006).

Anti-cancer properties have been reported in ginsenosides $\mathrm{Rb} 1, \mathrm{Rb} 2$ and $\mathrm{Rc}$ because of their ability to inhibit tumor angiogenesis and metastasis (Mochizuki et al. 1995; Chang 2003). Ginsenoside Re has also been reported to have anti-diabetic and anti-hyperlipidemic properties (Attele et al. 2002; Xie et al. 2005; Cho et al. 2006). Despite all these research reports, use of ginsenosides has not been incorporated into conventional medicine, mainly due to lack of rigorous well controlled long-term clinical studies (Kitts and Hu 2000). However, a significant potential exist for their future use in conventional medicine to treat some of the debilitating diseases such as cancer, diabetes, amnesia, Parkinson's and Alzheimer's diseases. Nonetheless, use of ginseng as herbal remedy and supplement will likely continue to increase.

From here forward use of the term ginseng will only be referring to American ginseng. The root is the most used plant part of ginseng, and often its value and desirability among Asian consumers is influenced by many factors including its shape (Pritts 1995; Guo et al. 1995). Ginseng root has been classified into 3-5 morphotypes. 
Sokhansanj et al (1999) classified freshly dug roots into three morphotypes: "Pencil", "chunky", and "complex". A "Pencil" root resembles a carrot, it has a slender body and lacks major lateral roots; a "chunky" root has 3 to 4 large lateral roots giving a "human" shape to the root; and a "complex" root has a single central body with lateral roots giving a "chicken claw" or "spider" shape to the root. Roy et al (2003) classified dried roots into five categories: "chunky", "forked", "pencil (carrot)", "spider", and "fiber". Following this classification, a "chunky" root is described as bullet or bubble shaped; a "forked" root has a humanoid appearance, and a "pencil" root has a main tap root equal to or greater than $5 \mathrm{~cm}$ in length. "Spider" roots have no distinct tap root present or if present is less than $2 \mathrm{~cm}$ in length and have several secondary and tertiary roots radiating from main root. "Fiber" roots comprise of secondary and tertiary roots with diameters of $1-2 \mathrm{~mm}$ or less.

The classification by Roy et al (2003) is typical of that employed by wholesalers, who often prune and separate dried roots to attain different grades. For example 'fiber' roots are obtained through pruning of secondary and tertiary roots off of the main roots. A 'spider' root is a very rare morphotype of ginseng and is often times regarded as a variant of a 'chunky' or 'bullet' root. Therefore only three categories are truly representative of the common root morphotypes encountered in ginseng at the farm level; 'Forked' or 'Man-like' (ML) - a branched root exhibiting a humanoid shape; 'Chunky' or 'Bulb' (BLB) - compact, round or bullet shaped root; and 'Pencil/Carrot/Stick' (STK) - slender, elongated tap root without lateral roots (Figure 3.1). 
The underlying causes of variations in root morphotypes and consequent effects on ginsenoside profiles have not been extensively studied (Li 1997). Soil texture and bulk density have been attributed to influencing the shape of ginseng root (Li 1997; Park et al. 2005; Roy et al. 2008). It is reported that coarse textured soils provide little resistance to root growth especially in the seeding year resulting in less desirable STK shaped roots, and that roots grown in loam soil are shorter and rounder than those grown in sand soil (Roy et al. 2008). Increasing bulk density (BD) has been reported to result in roots that are shorter, fatter, with wide mid-sections (Roy et al. 2008). Whereas soil texture and BD may play a role in determining root shape, the effect of underlying genetics cannot be discounted given that this species has been reported to be genetically heterogeneous, and in the light that influence of genotype on root morphology has been documented on Radish (Rhapanus sativus L.) (Bai et al. 1999, Bohem et al. 1999; Schluter and Punja 2002; Cruse-Sanders and Hamrick 2004; Grubbs and Case 2004; Iwata et al. 2004; Tsuro et al. 2007).

Variation in levels of total ginsenosides among ginseng roots are frequently reported in literature, and this has been attributed to several factors including ecotype (Lui and Staba 1980; Betz et al. 1984), age (Court et al. 1996; Smith et al. 1996), soil fertility and environmental conditions (Li et al. 1996; Li and Mazza 1999), population and genotype (Assinewe et al. 2003; Lim et al. 2005; Schlag and McIntosh 2006). Also variations in individual ginsenosides have been observed within and among populations, and these variations have been attributed to genotype variability (Lim et al. 2005; Schlag and McIntosh 2006). Rg1, Re, and Rb1 are often the most abundant saponins in 
American ginseng root and therefore have been extensively investigated for their medicinal properties (Murphy and Lee 2002). Recently it has been noted that ginsenosides $\operatorname{Rg} 1$ and $\operatorname{Re}$ are inversely related in ginseng, and variation in their profiles is evident even within a single population (Lim et al. 2005; Schlag and McIntosh 2006). The term chemotype has been used to describe the inverse relationship between $\operatorname{Rg} 1$ and Re ginsenosides (Schlag and McIntosh 2006). Roots with low Rg1 but high Re content are described as "low Rg1/high Re chemotype" - which is commonly observed in American ginseng, whereas roots with high Rg1 but low Re content are described as "high Rg1/low Re chemotype" - which is rarely observed.

Despite numerous reports on ginsenoside content and profiles in ginseng root, there has been less focus on relative abundance of individual ginsenosides among different root morphotypes. There are no known cultivars of ginseng (Schluter and Punja 2002), and this has been largely attributed to general lack of breeding direction for medicinal plants (Hong et al. 2005). Long held biased consumer preference for different root morphotypes coupled with research findings about pharmacological properties of individual ginsenosides will help set breeding targets for ginseng. In this study the influence of underlying genotypes on root morphotype was assessed, and frequency distribution of the three morphotypes (ML, BLB, and STK) among ginseng population was tabulated. Also the contents of six main ginensosides (Rg1, Re, Rb1, Rc, Rb2, and Rd) among the three root mophotypes were compared, with particular attention drawn to variations of $\mathrm{Rg} 1, \mathrm{Re}$ and $\mathrm{Rb} 1$ ginsenosides profiles. Lastly, potential economic implication for the grower who may opt to sort out his mixed batch of harvested roots to 
different morphotypes before selling as a way to leverage return for his crop was evaluated.

\section{Materials and Methods}

Greenhouse study - Stratified seeds were purchased from multiple sources (growers), and on June 2009, they were planted in the greenhouse in a regular Sunshine mix 1 growing medium (SUNGRO Horticulture, Seba Beach, Canada). The plants were grown under shade utilizing 55\% shade cloth and were watered once daily. After three months the roots were harvested, cleaned and sorted into different morphotypes. Frequency distribution of the three morphotypes was calculated.

Plant materials for ginsenoside analysis - Fresh roots aged 4, 8 and 10 years from woods cultivated American ginseng were collected from a single farm in western Maryland. These roots were grown under similar environmental conditions in raised soil beds of similar soil composition. Root ages were provided by the grower and were confirmed in our laboratory by counting the scars on the rhizome which typically indicates the root's age. From each age group, roots were separated into three distinct morphotypes (ML, BLB, and STK) and frequency distribution of each morphotype was calculated. The roots were then washed under running tap water, blotted dry with paper towels, and placed in individual paper bags. The roots were freeze dried for $72 \mathrm{hrs}$ and then ground into a fine powder to pass through $1 \mathrm{~mm}$ mesh in a Thomas ${ }^{\circledR}$ model 4 Wiley mill (Thomas Scientific, Swedensboro, NJ). Between each sample the mill was 
thoroughly cleaned to eliminate cross contamination between samples. Ground samples were stored at room temperature until needed for ginsenoside extraction.

Chemicals - All solvents were of high-performance liquid chromatography (HPLC) grade and were purchased from Fisher Scientific (Pittsburgh, PA). The water used for extraction and HPLC analysis was ultra purified by Milli-Q ${ }^{\circledR}$ water purification system, (Millipore, Billerica, MA). Ginsenoside standards kit containing 5 mg each of Rb1, Rb2, Rc, Rd, Re, and Rg1 (purity > 99\%) was purchased from Indofine Chemical Company, Inc. (Hillsborough, NJ). All solvents were filtered through a $0.45 \mu \mathrm{m}$ Autovial® PVDF membrane filters (Whatman, Inc., Clifton, NJ) before use.

Supplies $-0.45 \mu \mathrm{m}$ filter Centrifuge tubes (Spin- $\mathrm{X}^{\circledR} 8162$, Corning Inc., Corning, NY) and $10 \mathrm{ml}$ glass vials were purchased from Fisher Scientific (Pittsburg, PA), $300 \mu 1$ Polyvials with caps were purchased from Waters, Inc. (Milford, MA).

Extraction of ginsenosides - Our extraction method was based on that of Schlag and McIntosh (2005) with some modifications. One hundred milligrams of root powder was transferred into $10 \mathrm{ml}$ glass vials with vented cap, and $5 \mathrm{ml}$ of $80 \% \mathrm{MeOH}$ was added, briefly mixed by vortexing and the mixture was incubated in a water bath at $70^{\circ} \mathrm{C}$ with constant sonication for $1 \mathrm{hr}$. Thereafter, the vials were centrifuged for 5 minutes at 2000 rcf using a Mistral 3000i centrifuge (Curtin Matheson Scientific, Inc., Houston, TX), and the supernatant was transferred into clean tubes. The residues were re-extracted once and the supernatants from both extractions were combined and reduced to dryness under a 
stream of $\mathrm{N}_{2}$ at $38^{\circ} \mathrm{C}$, and then re-suspended in $2 \mathrm{ml}$ solvent of 20:20:60 (MeOH: $\mathrm{ACN}$ : $\mathrm{H}_{2} \mathrm{O}$ ). The concentrated extracts were filtered through $0.45 \mu \mathrm{m}$ membrane filters into clean $2 \mathrm{ml}$ polypropylene tubes (Spin- $\mathrm{X}^{\circledR} 8162$, Corning Inc., Corning, NY) and stored at $4^{\circ} \mathrm{C}$ until HPLC analysis $(<24 \mathrm{hrs})$. All root extractions were done in triplicates.

HPLC instrumentation and analysis - The HPLC system used was a Waters model 2695 Alliance separation module (Milford, MA) equipped with a PDA detector (Waters 996 PDA), an in-line degasser, an auto sampler, and Waters Millennium 32 software. The separation was carried out in Atlantis ${ }^{\circledR} \mathrm{T} 3$ column $(5 \mu \mathrm{m}, 250 \times 4.6 \mathrm{~mm})$ with Atlantis ${ }^{\circ}$ T3 guard cartridge $(5 \mu \mathrm{m}, 4.6$ x $20 \mathrm{~mm})$ (Waters, Inc., Milford, MA). The UV spectrum for ginsenosides was recorded at $203 \mathrm{~nm}$. For HPLC analysis, a $20 \mu 1$ sample or standard was injected into the column and eluted at room temperature at a constant flow rate of $1.2 \mathrm{ml} / \mathrm{min}$. The mobile phase used for separation consisted of solvent $\mathrm{A}$ (100\% water), and solvent B (100\% acetonitrile). A mobile phase gradient was based on that of Wang et al (2006) with slight modifications: From $0-20 \mathrm{~min}, 80 \%$ A and 20\% B; from 20 - 29 min, 74\% A and 26\% B; from $29-43$ min, 66\% A and 34\% B; from 43 - $47 \mathrm{~min}, 64 \% \mathrm{~A}$ and $36 \% \mathrm{~B}$; from $47-54 \mathrm{~min}, 57 \% \mathrm{~A}$ and $43 \% \mathrm{~B}$.

Serial concentration dilutions were made for individual standards and were injected into HPLC, and corresponding peak areas for each standard concentration were used to make standard curves (peak areas were averages of three runs per standard concentration). The standards were prepared using the same solvent as samples. All six ginsenoside standards were combined and $20 \mu 1$ of the mixture was injected to obtain 
retention times for respective ginsenosides. Presence of individual ginsenosides in each sample was confirmed by presence of peaks at retention times corresponding to those obtained from mixed standards. Repeatability was determined by injecting samples thrice per day (intraday variability) with samples ordered randomly in each run, whereas precision of measurement was determined by analyzing samples on three different days (interday variability) with relative standard deviation of $<5 \%$ of retention time determined as acceptable measurement variability attributable to instrument drift. Individual ginsenoside content in each sample were calculated using their respective standard curves $\left(r^{2}>0.99\right)$.

\section{Experimental design and statistical analysis of ginsenosides - The experiment was} designed as a randomized complete block. The experimental unit was an entire root. Since the roots were of different ages, blocking was used to reduce age effects since past research has demonstrated that age has a significant effect on ginsenosides content (Tani et al. 1981; Court et al. 1996). There were nine replicates for each morphotype in 4 and 8 year old roots, and six replicates for each morphotype in 10 year old roots. Our sample size was limited by the difficulty of obtaining the three distinct root morphotypes of equal age in the same population in order to ensure a more uniform comparison. Statistical analysis was done using the GLM procedure in SAS (SAS Institute, Inc., Cary, NC). When the dependent variable (individual or total ginsenoside) was significant at $\alpha=0.05$, Tukey's multiple comparison test was used to ascertain the differences. 


\section{Results and Discussion}

Greenhouse study - After just three months of growth, harvested root showed distinct morphotypes typical of those exhibited by older roots (Figure 3.1). Given that the growing medium was of uniform composition, devoid of any hard objects that may alter growth patterns of the roots and was of uniform bulk density, the variation of root morphotypes then would be attributed to underlying genetics. The relative abundance of morphotypes was 16\% ML, 24\% BLB, and 60\% STK (n=353). Similar frequency distributions of morphotypes were observed in roots sampled from 8 year old field grown ginseng: 14\% ML, 20\% BLB, and 66\% STK (n=102).

Chromatograms and ginsenosides content - Representative chromatograms of samples and standards are shown in Figure 3.2. All six ginsenosides were present in all root samples that were analyzed. Roots showed two distinct Rg1/Re profiles (low Rg1/ high Re, and high Rg1/low Re) (Figure 3.2). These profiles were not exclusive to any root morphotype, however, low Rg1/high Re profile was consistently exhibited by ML morphotypes, whereas BLB and STK morphotypes exhibited both profiles (Figure 3.3). The proportion of roots with low Rg1/high Re profile was 50 and 62.5\% in BLB and STK morphotypes respectively. There was no correlation between age and Rg1 or Re concentration across all morphotypes (Figure 3.3), but concentration of ginsenoside Rb1 mostly increased with age (Figure 3.4), and was positively correlated with total ginsenosides (Figure 3.6). 
Total ginsenoside content (sum of six quantified ginsenosides) ranged from $0.94-$ $3.47 \% \mathrm{w} / \mathrm{w}$ for ML morphotypes, $1.12-2.55 \% \mathrm{w} / \mathrm{w}$ for BLB morphotypes, and 1.37 $2.65 \% \mathrm{w} / \mathrm{w}$ for STK morphotypes. There was a gradual increase in total ginsenoside with age independent of morphotype except for STK morphotype where 4 and 8 year old roots had almost equal amount of total ginsenosides despite the age difference (Figure 3.5). This observation that ginsenoside content increases with age is consistent with other published reports (Tani et al. 1981; Court et al. 1996; Smith et al.1996) and further reaffirms the widely accepted practice of harvesting older roots since they have more ginsenosides content than younger roots $(<4$ years) and therefore regarded to be more potent.

Comparisons between root morphotypes of same age showed that total ginsenoside content was significantly higher in 10 year old roots of ML morphotype than those of BLB and STK morphotypes (Figure 3.5). In 8 year old roots there was no significant difference in total ginsenoside content except for roots of STK morphotype, whereas in 4 year old roots there was no significant difference in total ginsenoside across morphotypes (Figure 3.5). Comparison of individual ginsenoside content among root morphotypes showed significant differences on levels of $\mathrm{Rg} 1, \mathrm{Re}$, and $\mathrm{Rb} 1$ ginsenosides but not on those of Rc, Rb2, and Rd ginsenosides (Figure 3.6). The most abundant ginsenoside of protopanaxadiol group in all morphotypes was Rb1, which accounted for $51.60,51.08$, and $45.25 \%$ of the total ginsenoside contents in ML, BLB and STK morphotypes respectively (Table 3.1). These results agree with other previous published reports on field grown and in vitro transformed $P$. quinquefolius roots (Li et al. 1996; 
Mallol et al 2001; Schlag and McIntosh 2006). Rb2 was the least abundant ginsenoside of the protopanaxadiol group accounting for $1.83,2.69$, and $3.91 \%$ of total ginsenoside contents in ML, BLB, and STK morphotypes respectively (Table 3.1).

Ginsenosides of the protopanaxatriol group ( $\mathrm{Rg} 1$ and $\mathrm{Re})$ were inversely related in all three morphotypes (Figure 3.3), and Re was the main ginsenoside of this group constituting $29.68,18.82$, and $22.91 \%$ of the total ginsenosides content in ML, BLB, and STK morphotypes respectively (Table 3.1), which is in accordance with previous studies (Lim et al. 2005; Schlag and McIntosh 2006). Rg1 and Re accounted for 2.74 and $29.68 \%$ of total ginsenoside respectively in ML morphotype, 11.83 and $18.83 \%$ in BLB morphotype, and 10.06 and $22.91 \%$ in STK morphotype (Table 3.1). The most notable finding from this study was that relative abundance of $\mathrm{Rg} 1$ was low in roots of ML morphotypes (2.74\%); almost five fold less compared to that in roots of BLB or STK morphotypes. In a sharp contrast, Re content was significantly higher in ML roots than in BLB or STK roots, with a fold difference in content of 1.3 over BLB roots and 1.5 over STK roots (Table 3.1).

Ginsenoside Re is formed after enzymatic addition of two rhamnose residues to $\operatorname{Rg} 1$ (Gubar et al. 1997). In this study, comparisons of Re and $\operatorname{Rg} 1$ contents across root morphotypes showed that the ratios of $\operatorname{Re} / \operatorname{Rg} 1$ ginsenosides were $10,1.5$, and 2.3 in ML, BLB, and STK roots respectively. Based on these ratios it can be inferred that the enzymatic activities involved in adding sugar molecules on $\operatorname{Rg} 1$ to form Re may be higher in ML roots than in BLB or STK roots. However, further studies on enzymatic 
activity among root morphotypes will need to be conducted to confirm this hypothesis and determine if that activity is genetically predisposed.

Overall, roots of ML morphotypes had significantly higher total ginsenosides (adjusted mean, $2.19 \pm 0.07 \% \mathrm{w} / \mathrm{w})$ than those of BLB morphotype $(1.86 \pm 0.07 \% \mathrm{w} / \mathrm{w})$ or STK morphotype $(1.79 \pm 0.07 \% \mathrm{w} / \mathrm{w})$, however there was no significant difference in total ginsenosides content between roots of BLB and STK morphotypes (Figure 3.6). Ginsenosides are reported to be located in the periderm and cortex regions of the root (Tani et al. 1981). Given that previous studies have reported that ginseng root hairs contain high ginsenosides content than main roots (Tani et al. 1981; Christensen et al. 2006), then the significantly higher total ginsenoside content in ML roots could be due to the more abundance of root hairs and lateral roots in this morphotype than in the other morphotypes. However, this could also be due to underlying genetics, given the substantial genetic variability of this species.

Ginsenoside contents of roots analyzed in this study were relatively lower compared to those previously reported $(3-6 \% \mathrm{w} / \mathrm{w})$ for cultivated $P$. quinquefolius in North America (Court et al. 1996; Li et al. 1996; Assinewe et al. 2003). However, this results are consistent with those reported for Maryland populations $(2.3 \% \mathrm{w} / \mathrm{w})$ (Schlag and McIntosh 2006), and New York populations (2.5\% w/w) (Lim et al. 2005) for the same age range $(4-10 y r s)$. Variability in ginsenoside content is expected for $P$. quinquefolius given that this species is genetically heterogeneous and naturally grows in a 
broad geographic region with distinctly different ecological conditions (Bai et al. 1997; Boehm et al. 1999; Assinewe et al. 2002).

Economic analysis - Biased quality assessment and pricing of ginseng in the market based on root morphotype has significant implications on ginseng returns. Therefore the expected economic return from growing and marketing of select root morphotypes potentially could guide cultivation and marketing decisions for a ginseng grower. A simple economic analysis was carried out to evaluate potential economic implications of sorting roots into respective morphotypes after harvest as a way to increase returns for the grower. The analysis calculations are based on estimates of root yield per acre and frequency distribution of each root morphotype in a mixed batch (MXD) based on their respective mean frequency distributions as observed from both the greenhouse study and field tabulations (15\% ML, 22\% BLB, and 63\% STK). Using total ginsenosides as a criterion for quality, returns due to sorting has been assessed at two different price premium mark ups (5\% and 30\%) for ML roots over the average price for a pound of mixed root morphotypes (\$60) of woods cultivated ginseng.

Yield of roots per acre based on different planting densities is shown in Table 3.2. The yield calculations are based on planting density of $6 \times 6$ inches which was utilized on the farm where roots samples were collected. Under these specifications number of roots per acre will be 238,032 after assuming a $20 \%$ root loss (Table 3.2). Root weight yields per acre for MXD root morphotype batch (current sale system) and sorting to different morphotypes (for value addition) are shown in Table 3.3. Estimated gross value per acre 
of production under current selling system (MXD) is \$238,967 (Table 3.3), and returns from sorting roots to different morphotypes (value addition) before selling after factoring in sorting cost ( $\$ 1,000$ per acre) with $5 \%$ and $30 \%$ price premium mark up for ML roots will be $\$ 2,112$ and $\$ 17,672$ respectively (Table 3.3). However if labor cost for sorting rises, the returns from 5\% price mark up for ML morphotype over MXD will break even if sorting labor cost reaches $\$ 3,112$ per acre. Sorting roots to respective morphotypes in spite of increasing the premium of ML morphotype, will more likely lower the value of BLB and STK roots. If that occurs, then sorting will not result in any increase in revenue return even at the lowest sorting labor cost. However, in future, if breeding efforts will yield cultivars of a desirable morphotype (ML), then revenue returns from growing high quality cultivars (ML morphotype) could be substantial (Table 3.4).

\section{Conclusions}

In this study, it has been demonstrated that variation in root morphotypes of ginseng is possibly influenced by underlying genotype. Majority of roots in ginseng population are of STK morphotype, whereas ML morphotype has the lowest occurrence. The presented results have shown that roots of ML morphotype have higher total ginsenoside content than roots of BLB or STK morphotypes, but there is no significant difference in total ginsenoside content between BLB and STK roots. Using total ginsenoside as a criterion for assessing quality of roots, then roots of ML morphotypes would be regarded to be of higher quality than those of BLB or STK morphotypes. With regard to major ginsenosides, the concentrations of Re and Rb1 are higher in roots of ML morphotype compared to those of BLB and STK morphotypes, however Rg1 is lowest in roots of ML 
morphotype. This research has shown that the ML morphotype predominantly exhibit low Rg1/high Re profile, whereas BLB and STK morphotypes exhibit both low Rg1/high Re, and high $\operatorname{Rg} 1 /$ low Re profiles.

Currently there are no cultivars for American ginseng (Schluter and Punja 2002), mainly due to lack of breeding direction for medicinal plants in general (Hong et al 2005). However with studies reporting various pharmacological activities of individual ginsenosides, coupled with reports on variation of ginsenoside profiles and concentration among specific roots morphotypes, there are potential breeding targets for American ginseng. For example Re is reported to be very effective against diabetes and lowering cholesterol (Attele et al. 2002; Xie et al. 2005; Cho et al. 2006), therefore cultivars with high Re levels (ML roots) could be desirable. On the other hand, Rg1 is reported to have neuroprotective properties (Liao et al. 2002), so cultivars with high concentration of $\operatorname{Rg} 1$ (BLB and STK morphotypes) could be targeted.

Estimates from the economic analysis show that under the current production system (no selected cultivars), sorting roots to different morphotypes as a strategy to leverage returns will result in revenue increase, however the level of return due to sorting will be dependent upon the cost of sorting and price premium mark up the grower will assign to high quality roots. Looking forward, the prospects for improving returns are substantial for American ginseng if cultivars become available. 


\section{Literature Cited}

Assinewe, V. A., B. R. Baum, D. Gagnon, J. T. Arnason. 2003. Phytochemistry of wild populations of Panax quinquefolius L. (North American ginseng). Journal of Agricultural and Food Chemistry 51: 4549-4553.

Attele, A. S., J. A. Wu, C. S. Yuan. 1999. Ginseng pharmacology: multiple constituents and multiple actions. Biochememical Pharmacology 58: 1685-1693.

Attele, A. S., Y. P. Zhou, J. T. Xie, J. A. Wu, L. Zhang, L. Dey, W. Pugh, P. A. Rue, K. S. Polonsky, and C. S. Yuan. 2002. Antidiabetic effects of Panax ginseng berry extract and the identification of an effective component. Diabetes 51: 1851-1858.

Bai, D., J. Brandle, and R. Reeleder. 1997. Genetic diversity in North American ginseng (Panax quinquefolius L.) grown in Ontario detected by RAPD analysis. Genome 40: $11-115$.

Betz, J. M., A. H. DerMarderosian, and T. Lee. 1984. Continuing studies on ginsenoside products by TLC and HPLC, II. Proceedings of the $6^{\text {th }}$ Annual North American ginseng conference. J.T.A Proctor, (eds). University of Guelph, Guelph, Canada. pp. 65-83.

Boehm, C. L., H. C. Harrison, G. Jung, and J. Nienhuis. 1999. Organization of American and Asian ginseng germplasm using randomly amplified polymorphic DNA (RAPD) markers. Journal American Society Horticulture Science 124: 252-256.

Brevoort, P. 1998. The blooming U.S. botanical market: a new overview. Herbalgram 44: 33-46. 
Carpenter, S. G., and G. Cottam. 1982. Growth and reproduction of American ginseng (Panax quinquefolius) in Wisconsin, USA. Canadian Journal of Botany 60: $2692-2696$.

Catling, P. M., E. Haber, and E. Small. 1994. Poorly known economic plants of Canada: ginseng_Panax quinquefolius L. Canadian Botanical Association Bulletin 27: $70-72$.

Chang, Y. S., E. K. Seo, C. Gyllenhaal, and K. I. Block. 2003. Panax ginseng: a role in cancer therapy?. Integrative Cancer Therapies 2: 13-33.

Charron, D., and D. Gagnon. 1991. The demography of northern populations of Panax quinquefolium (American ginseng). Journal of Ecology 79: 431-445.

Cheng, L. and P.D. Mitchell. 2009. Status of the Wisconsin Ginseng Industry. Department of Agricultural and Applied Economics, University of Wisconsin-Madison, Madison, Wisconsin, USA.

Cho, W. C. S., W.-S. Chung, S. K. W. Lee, A. W. N. Leung, C. H. K. Cheng, and K. K. M. Yue. 2006. Ginsenoside Re of Panax ginseng possesses significant antioxidant and antihypelipidemic efficacies in streptozotocin-induced diabetic rats. European Journal of Pharmacology 550: 173-179.

Christensen, L. P., M. Jensen, and U. Kidmose. 2006. Simultaneous determination of ginsenosides and polyacetylenes in American ginseng root (Panax quinquefolium L.) by high performance liquid chromatography. Journal of Agricultural and Food Chemistry 54: 8995-9003.

Duke, J. 1989. Ginseng: A concise handbook. Reference Publication, Inc. Algonac. Mississippi.USA. 
Goldstein, B. 1975. Ginseng: Its history, dispersion, and folk tradition. American Journal of Chinese Medicine 3: 223-234.

Gubar, S. I., T. P. Gulko, and V.A. Kunakh. 1997. Growth and glycoside accumulation in ginseng callus tissue culture under long-term action of exogenous phytohormones. Russian Journal of Plant Physiology 44: 83-89.

Guo, Y. P., W. G. Bailey, and K. B. van Dalfsen. 1995. North American ginseng (Panax quinquefolium L.) root grading. In W.G. Bailey, C. Whitehead, J.T.A. Proctor, and J. T. Kyle, eds, pg 380-389. The challenges of the $21^{\text {st }}$ century. Proceedings of the International Ginseng Conference-Vancouver 1994, Simon Frazer University, Burnaby, BC, Canada.

Hu, Y. S., L. Rudenberg, and P. D. Tredici. 1980. Studies of American Ginseng. Rhodora 82: 627-638.

Hoareau, L. and E. J. DaSilva. 1999. Medicinal plants: a re-emerging health aid. Electronic Journal of Biotechnology 2(2): 56-70.

Hong, Y. Q. D., A.J. Lau, C. L. Yeo, X. K. Liu, C. R. Yang, H. L. Koh, and Y. Hong. 2005. Genetic diversity and variation of saponin contents in Panax notoginseng root from a single farm. Journal of Agricultural and Food Chemistry 53: 84608467.

Iwata, H., S. Niikura, Y. Takano, and Y. Ukai. 2004. Genetic control of root shape at different growth stages in Radish (Raphanus sativum L.). Breeding Science 54: $117-124$

Ki, Y. N., S.-R. Ko, and K.-J. Choi. 1998. Relationship of saponin and non-saponin for the quality of ginseng. Journal of Ginseng Research 22: 272-283. 
Kim, H., B. Sung, J. Park, C. Sim, Y. Kim, and S. Lee. 2005. Application of neutron radiography and tomography for analysis of root morphology of growing ginseng. Proceedings of the International Symposium on Research Reactor and Neutron Science - In Commemoration of the $10^{\text {th }}$ Anniversary of HANARO- Daejeon, Korea.

Kimura, Y., M. Sumiyoshi, K. Kawahira, and M. Sakanaka. 2006. Effects of ginseng saponins isolated from Red ginseng roots on burn wound healing in mice. British Journal of Pharmacology 148: 860-870.

Kitts, D. D. and C. Hu. 2000. Efficacy and safety of ginseng. Public Health Nutrition 3: $473-485$.

Kitts, D. D., A. N. Wijewickreme, and C. Hu. 2000. Antioxidant properties of a North American ginseng extract. Molecular Cellular Biochemistry 203: 1-10.

Konsler, T. R., and J. E. Shelton. 1990. Lime and phosphorus effects on American Ginseng I: Growth, soil fertility, and root tissue nutrient status response. Journal of American Society of Horticultural Sciences 115: 570-574.

Konsler, T. R., S. W. Zito, J. E. Shelton, and E. J. Staba. 1990. Lime and phosphorus effects on American Ginseng II: Root and leaf ginsenoside content and their relationship. Journal of American Society of Horticultural Sciences 115: 575580.

Lewis, W. H. 1988. Re-growth of a Decimated Population of (Panax quinquefolium) in a Missouri Climax Forest. Rhodora 90: 1-5.

Lewis, W., and V. Zenger. 1982. Population dynamics of American ginseng (Panax quinquefolium) Araliaceae. American Journal of Botany 69 (9): 1483-1490. 
Lewis, W., and V. Zenger. 1983. Breeding systems and fecundity in the American ginseng, Panax quinquefolium (Araliaceae). American Journal of Botany 70 (3): $466-468$.

Li, T. S. C. 1997. Effects of seedling depth and of soil texture texture on seedling emergence and root shape of American ginseng. Korean Journal of Ginseng Research 21: 115-118.

Li, T. S. C., G. Mazza, A. C. Cottrell, and L. Gao. 1996. Ginsenosides in roots and leaves of American ginseng. Journal of Agricultural and Food Chemistry 44: $717-720$

Li, T. S. C., and G. Mazza. 1999. Correlations between leaf and soil mineral concentrations and ginsenoside contents in American ginseng. HortScience 34: $85-87$.

Liao, B., H. Newmark, and R. Zhou. 2002. Neuroprotective effects of ginseng total saponin and ginsenoside Rb1 and $\operatorname{Rg} 1$ on spinal code neurons in vitro. Experimental Neurology 173: 224-234.

Lim, W., K. W. Mudge, and F. Vermeylen. 2005. Effects of population, age, and cultivation methods on ginsenoside content of wild American ginseng (Panax quinquefolium). Journal of Agricultural and Food Chemistry 53: 8498-8505.

Liu, C. X., and P. G Xiao. 1992. Recent advances in on ginseng research in China. $J$. Ethnopharmacol 36: 26-38.

Lui, J. H. C., and E. J. Staba. 1980. The ginsenosides of various ginseng plants and selected products. Journal of Natural Products 43: 340-346. 
Mallol, A., R. M Cusido, J. Palazon M. M. Bonfill, C. Morales, and M. T. Pinol. 2001. Ginsenoside production in different morphotypes of Panax ginseng transformed roots. Phytochemistry 57: 365-371.

Murphy, L. L. and T. J. Lee. 2002 Ginseng, sex behavior and nitric oxide. Annals of the New York Academy of Science 962: 372-377.

Mochizuki, M., Y.C. Yoo, K. Matsuzawa, K. Sato, I. Saiki, and S. Tonooka. 1995. Inhibitory effect of tumor metastasis in mice by saponins, ginsenoside-Rb2, 20(R)- and 20(S)- ginsenoside-Rg3 of red ginseng. Biological and Pharmaceutical Bulletin 18: 1197-1202.

Mook-Jung, I., H. S Hong, J. H. Boo, K. H. Lee, S. H. Yun, M. Y. Cheong, I. Joo, K. Huh, and M. W. Jung. 2001. Ginsenoside Rb1 and Rg1 improve spatial learning and increase hippocampal synaptophysin level in mice. Journal of Neuroscience Research 63: 509-515.

O’Hara, M., D. Kiefer, K. Farrell, and K. Kemper. 1998. A review of 12 commonly used medicinal herbs. Archives of Family Medicine 7: 523-53.

Park, H., B. D. Lee, and J.-M. Lee. 2005. Effect of soil condition on the root contraction of ginseng. Acta Horticulturae. 676: 155-160.

Persons, W. S. 1994. American ginseng: Green Gold. Bright Mountain Books, Inc. Asheville, North Carolina, USA.

Pierce, A. R. 2002. American ginseng (Panax quinquefolius): Biology, ecology, trade, social issues, and prospects for conservation. Case study of the Rainforest Alliance Sustainable Botanicals Program. New York, New York, USA. 
Pritts, K. D. 1995. How to find, grow, and use America's forest gold. Stackpole Books. Mechanicsburg, Pennsylvania, USA.

Proctor, J. T. A. 1996. Ginseng: Old crop, new directions. In: Janick J (ed) Progress in new crops. ASHS Press, Arlington, Virginia, USA.

Proctor J. T. A. and W. G. Bailey. 1987. Ginseng: Industry, botany, and culture. Horticulture Reviews 9: 187-236.

Radad, K., G. Gille, L. Liu, and W.D. Rausch. 2006. Use of ginseng in medicine with emphasis on neurodegenerative disorders. Journal of Pharmacological Science 100: 176-186.

Rahman M. and Z.K. Punja. 2005. Biochemistry of ginseng root tissues affected by rusty root symptoms. Plant Physiology and Biochemistry 43: 1103-1114.

Ren, G. and F. Chen. 1999. Simultaneous Quantification of Ginsenosides in American Ginseng (Panax quinquefolium) Root Powder by Visible/Near-Infrared Reflectance Spectroscopy. Journal of Agricultural and Food Chemistry 47: 27712775.

Robbins, C. S. 1998. American ginseng: The root of North America's medicinal herb trade. TRAFFIC North America Report. Number B347, Washington, D.C. USA.

Roy, R. C., R. Grohs, and R. D. Reeleder. 2003. A method for the classification by shape of dried roots of ginseng (Panax quinquefolius L.). Canadian Journal of Plant Science 83: 955-958.

Roy, R. C., B. R. Ball Coelho, R. D. Reeleder, A. J. Bruin, R. Grohs, P. H. White, and B. B. Capell. 2008. Effect of planting bed shape, mulch and soil density on root 
yield and shape in North American ginseng (Panax quinquefolius L.). Canadian Journal of Plant Science 88 (5): 937-949.

Schlag, E. M. and M. S. McIntosh. 2006. Ginsenoside content and variation among and within American ginseng (Panax quinquefolius L.) populations. Phytochemistry 67: 1510-1519.

Schlessman, M. 1985. Floral biology of American ginseng (Panax quinquefolium). Bulletin of the Torrey Botanical Club 112 (2): 129-133.

Schluter, C. and Z. K. Punja. 2002. Genetic diversity among natural and cultivated field populations and seed lots of American ginseng (Panax quinquefolius L.) in Canada. International Journal of Plant Science 163 (3): 427-439.

Schumann, D. R. and M. G. Thomas. 1993. Income opportunities in special forest products: Self help suggestions for rural entrepreneurs. Agricultural Information Bulletin (666). U.S. Department of Agriculture, Washington, D.C. USA.

Sengupta, S., S.-A. Toh, L. A. Sellers, J. N. Skepper, P. Koolwijk, H. W. Leung, H.-W. Yueng, R. N. S. Wong, R. Sasisekharan, and T.-P. D. Fan. 2004. Modulating angiogenesis: the yin and yang in ginseng. Circulation 110: 1219-1225.

Shen, L.and J. Zhang. 2003. Ginsenoside Rg1 increases ischemia-induced cell proliferation and survival in the dentate gyrus of adult gerbils. Neuroscience Letters 344: 1-4.

Shibata, S., O. Tanaka, J. Shoji, H. Saito. 1985. Chemistry and pharmacology of Panax. In: Wagner, H., H. Hikino, N. R. Farnsworth, (eds). Economic and Medicinal Plant Research Vol. 1 Academic Press, New York. 217-284. 
Smith, R. G., D. Caswell, A. Carriere, and B. Zielke. 1996. Variation in the ginsenoside content of American ginseng, Panax quinquefolius L., roots. Canadian Journal of Botany 74: 1616-1620.

Sokhansanj, S., W. G. Bailey, Y. P. Guo, K. B. van Dalfsen, and A. L. Oliver. 1999. Physical characteristics of North American ginseng (Panax quinquefolius L.) root. Canadian Society of Agricultural Engineering 41: 239-246.

Sumner, J. 2000. The natural history of medicinal plants. Timber Press, Portland, Oregon.USA.

Tani, T., K. Michinori, K. Tadahisa, H. Masayuki. H. Teruaki, and A. Shigaru. 1981. Histochemistry II. Ginsenoside in ginseng (Panax ginseng) root. Journal of Natural Products 44: 401-407.

Teng, R., C. Ang, D. McManus, D. Armstrong, S. Mau, and A. Bacic. 2003. Regioselective acylation of ginsenoside by Novozyme 435. Tetrahedron Letters 44: 5661-5664.

Tsuro. M., K. Suwabe, N. Kubo, S. Matsumoto, and M. Masashi. 2007. Maping of QTLs controlling root shape and red pigmentation in radish, Raphanus sativum L. Breeding Science 58: 55-61.

Vanisree, M., C. Y. Lee, S. F. Lo, S. M. Nalawade, C. Y. Lin, and H. S. Tsay. 2004. Studies on the production of some important secondary metabolites from medicinal plants by plant tissue cultures. Botanical Bulletin of Academia Sinica 45: $1-22$. 
Wang, C. Z., J. A. Wu, E. McEntee, and C. S. Yuan. 2006. Saponins composition in American ginseng leaf and berry assayed by high performance liquid chromatography. Journal of Agricultural and Food Chemistry 54: 2261-2266.

Wen, T. C., H. Yoshimura, S. Matsuda, J. H. Lim, and M. Sakanaka. 1996. Ginseng root prevents learning disability and neuronal loss in gerbil with 5-minute forebrain ischemia. Acta Neuropathologica 91: 15-22.

Xie, J-T., S. R. Mehendale, X. Li, R. Quigg, X. Wang, C.-Z. Wang, J.A. Wu, H. H. Aung, P. A. Rue, G. I. Bell, and C.-S. Yuan. 2005. Anti-diabetic effect of ginsenoside Re in ob/ob mice. Biochimica et Biophysica Acta 1740 (3): 319-325.

Zhang, B., S. Matsuda, J. Tanaka, N. Tateishi, N. Maeda, T.-C. Wen, H. Peng, and M. Sakanaka. 1998. Ginsenoside Rb1 prevents image navigation disability, cortical infarction, and thalamic degeneration in rats with focal cerebral ischemia. Journal of Stroke and Cerebrovascular Diseases 7 (1): 1-9.

Zou, K., S. Zhu, M. R. Meselhy, C. Tohda, and K. Komatsu. 2002. Dammarene type saponins from Panax japonicus and their neutrite outgrowth activity in SK-N-SH cells. Journal of Natural Products 65: 1288-1292. 

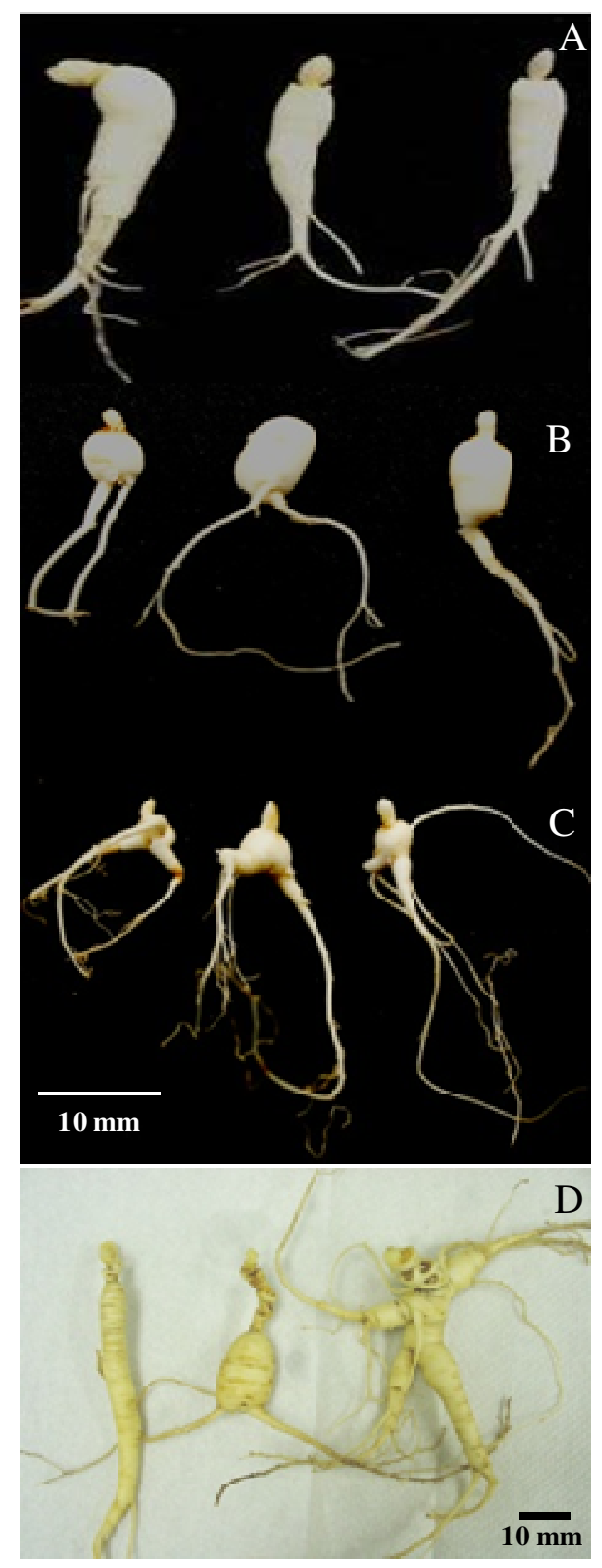

Figure 3.1. Different root morphotypes of Panax quinquefolius. A through C; 3 month old roots grown in regular Sunshine mix in a greenhouse: A) STK morphotype; B) BLB morphotype; C) ML morphotype; D) 8 year old woods cultivated roots showing all three morphotypes, left to right; STK, BLB and ML morphotypes respectively. The scale bar in $\mathrm{C}$ applies to all 3 month old roots. 


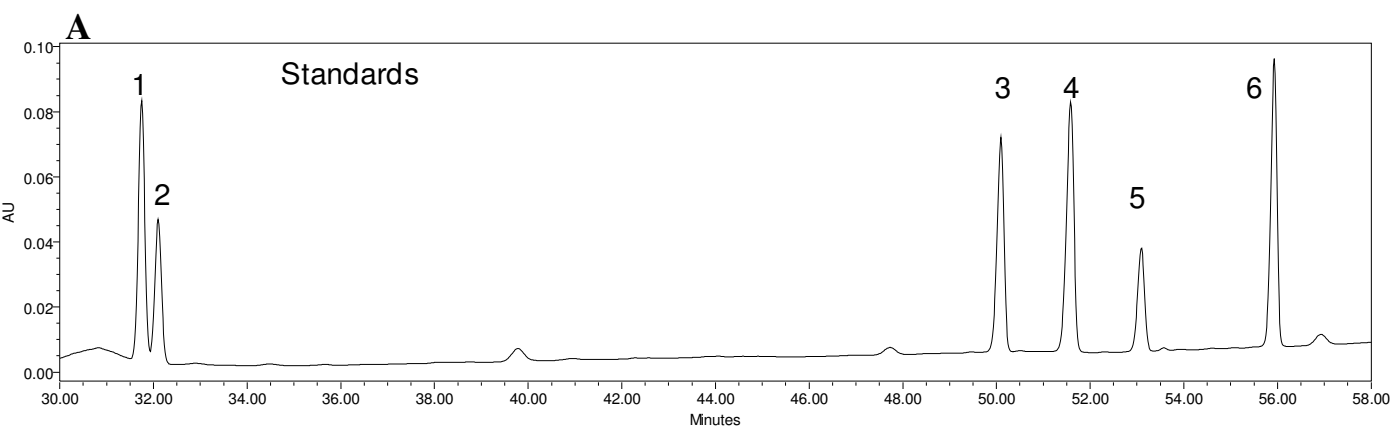

B
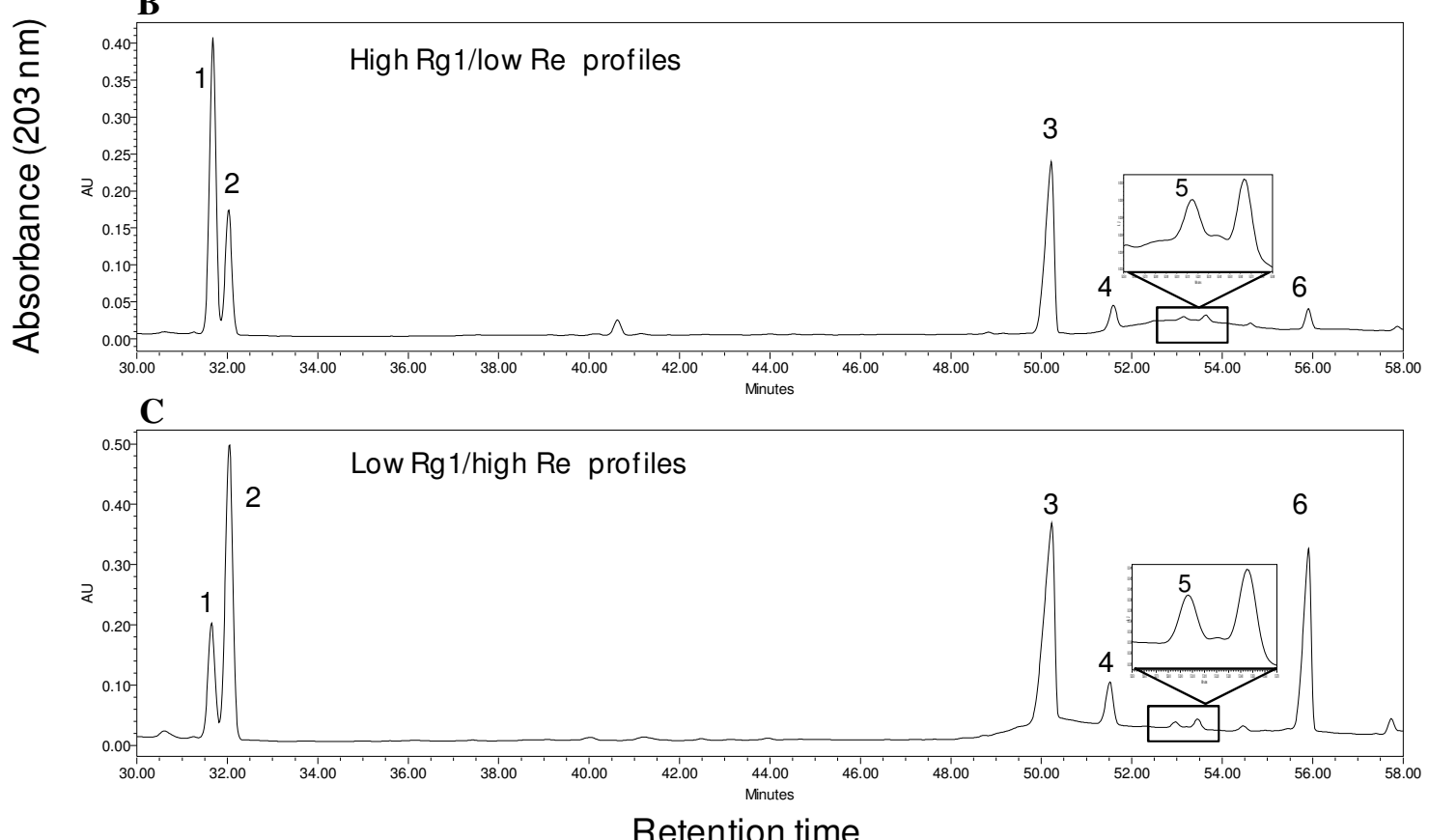

Figure 3.2. HPLC chromatograms of six ginsenosides in P. quinquefolius root samples: A) mixed standard solution; B) chromatograms showing high Rg1/low Re profile; C) low $\mathrm{Rg} 1 /$ high Re profile in root samples. Ginsenoside peaks; 1 (Rg1), 2(Re), 3(Rb1), 4(Rc), 5(Rb2), and 6(Rd). 
$\square \operatorname{Rg} 1 \square \operatorname{Re}$

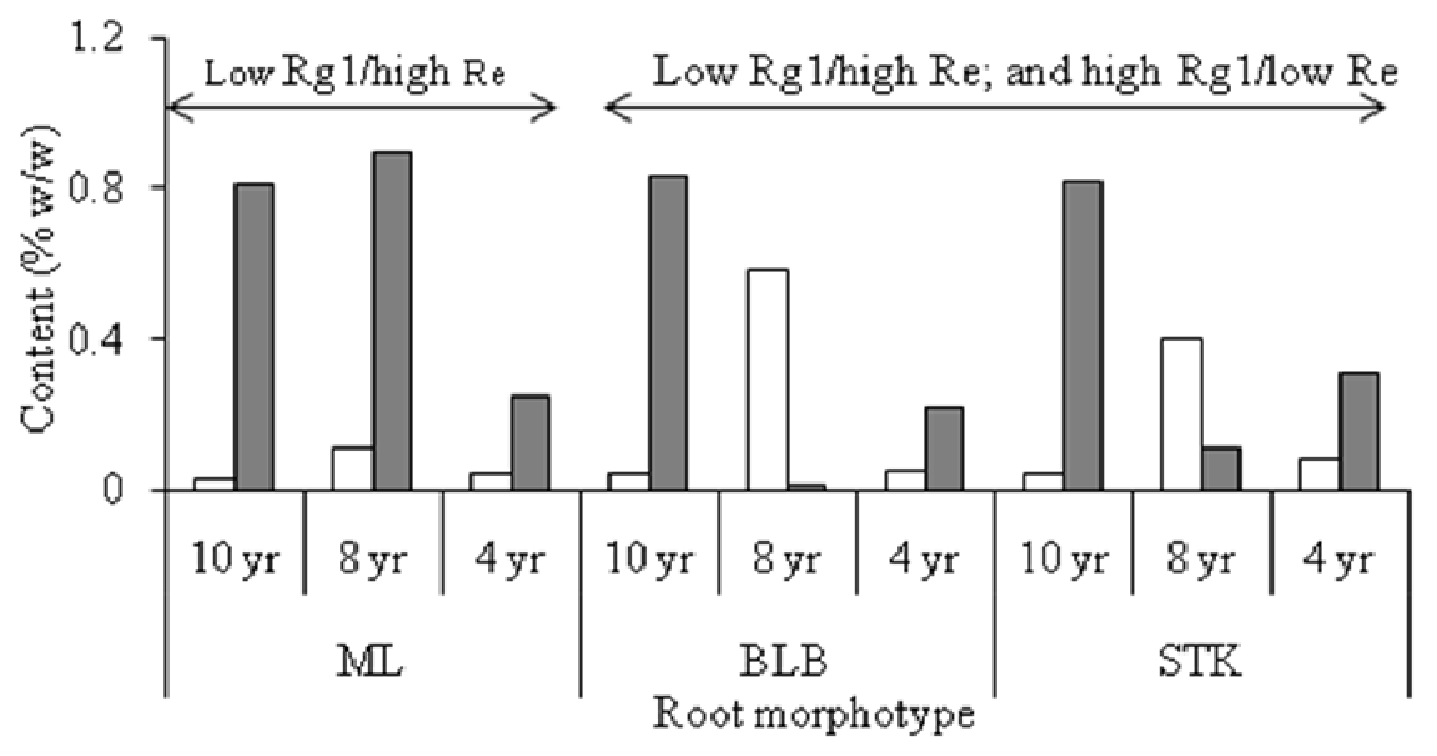

Figure 3.3. Profiles of ginsenoside $\mathrm{Rg} 1$ and $\mathrm{Re}$ in roots of $P$. quinquefolius of three different ages and morphotypes. 


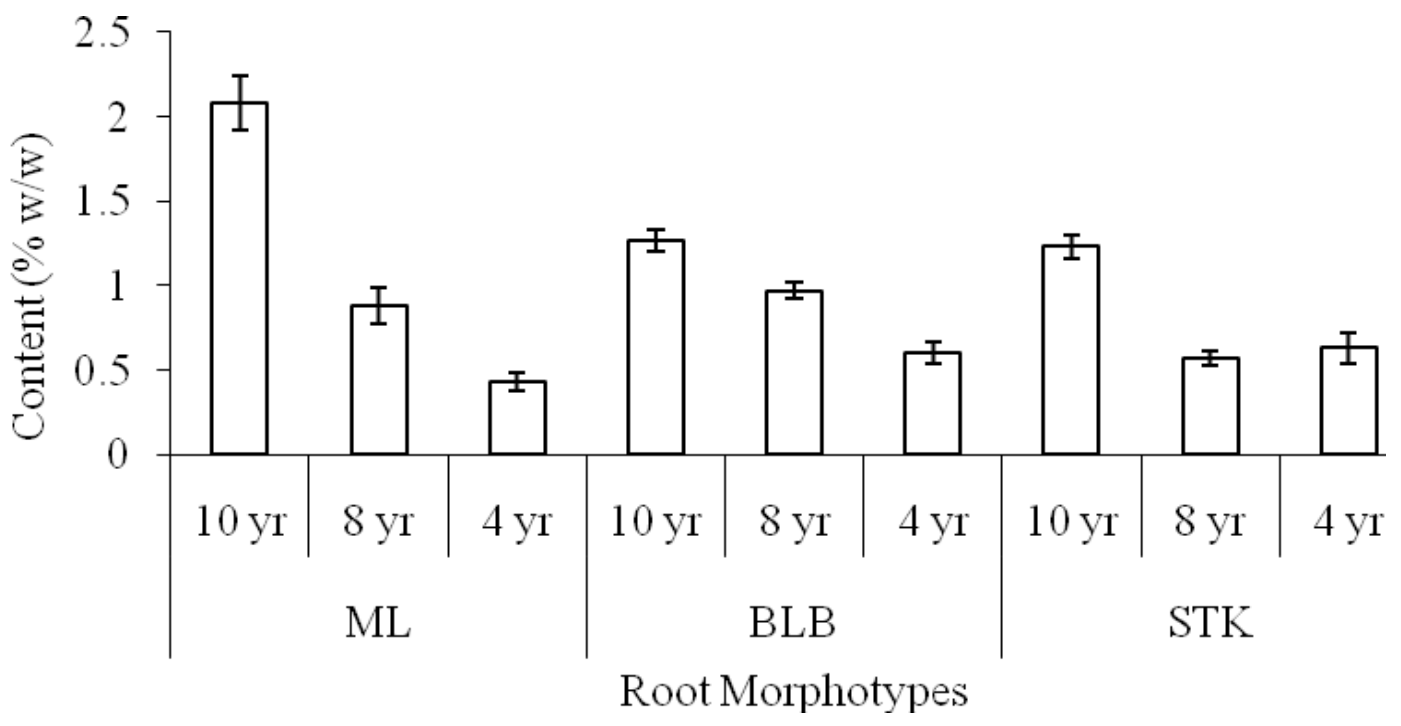

Figure 3.4. Concentration of ginsenoside $\mathrm{Rb} 1$ in roots of $P$. quinquefolius of three different ages and morphotypes. 


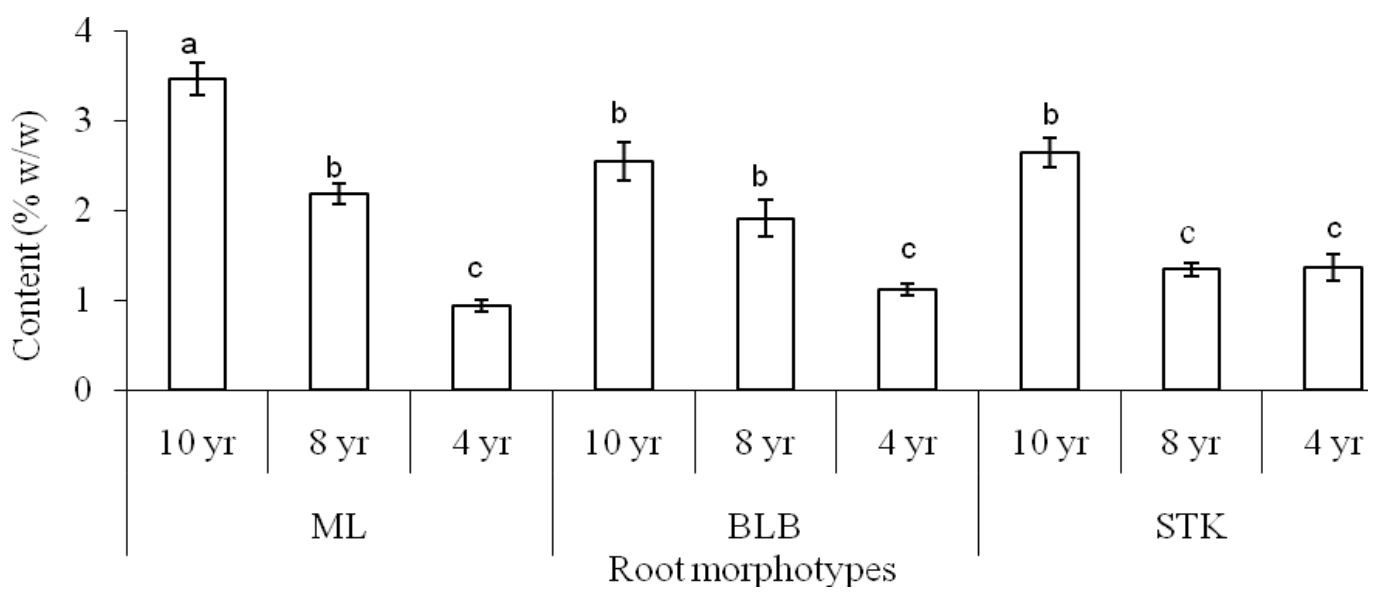

Figure 3.5. Comparison of total ginsenoside concentration (\% w/w) of P. quinquefolius roots by age and morphotype. Mean concentrations accompanied by same letter are not significantly different at $\alpha=0.05$ following Tukey's multiple comparison test. 


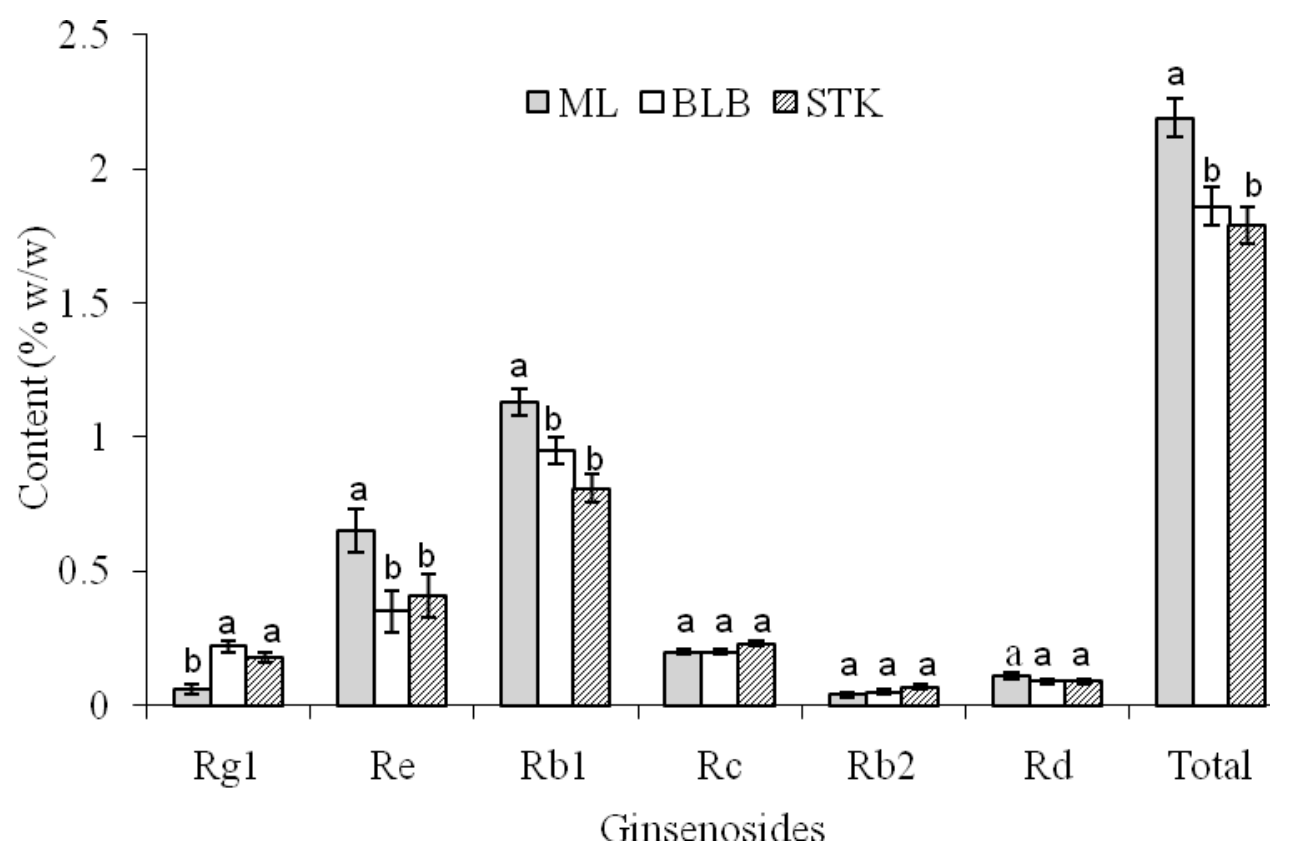

Figure 3.6. Concentration (Adjusted means \pm SEM) of six individual and total ginsenosides of different morphotypes of $P$. quinquefolius roots. Mean concentrations of individual and total ginsenosides accompanied by same letter are not significantly different among morphotypes at $\alpha=0.05$ following Tukey's multiple comparison test. 
Table 3.1. Relative abundance of individual ginsenosides to total content by root morphotype.

\begin{tabular}{lrrrrrr}
\hline \multirow{2}{*}{$\begin{array}{l}\text { Root } \\
\text { Morphotype }\end{array}$} & \multicolumn{6}{c}{ Individual ginsenosides \% of total $^{*}$} \\
\cline { 2 - 7 } & $\operatorname{Rg} 1$ & $\operatorname{Re}$ & $\mathrm{Rb} 1$ & $\mathrm{Rc}$ & $\mathrm{Rb} 2$ & \multicolumn{1}{c}{$\mathrm{Rd}$} \\
\hline & & & & & & \\
ML & 2.74 & 29.68 & 51.60 & 9.13 & 1.83 & 5.02 \\
BLB & 11.83 & 18.82 & 51.08 & 10.75 & 2.69 & 4.84 \\
STK & 10.06 & 22.91 & 45.25 & 12.85 & 3.91 & 5.03 \\
\hline
\end{tabular}

* Sum of the six ginsenosides quantified in this study (Rg1, Re, Rb1, Rc, Rb2, Rd) 
Table 3.2. Root yield per acre of $P$. quinquefolius under different planting densities

\begin{tabular}{|l|c|l|c|c|}
\hline $\begin{array}{l}\text { Spacing in } \\
\text { inches * }\end{array}$ & $\begin{array}{l}\text { Plants per } \\
\text { sq. foot }\end{array}$ & $\begin{array}{l}\text { Plants per } \\
\text { acre }\end{array}$ & $\begin{array}{l}\text { Plants per acre } \\
\text { Assuming 20\% root loss }\end{array}$ & $\begin{array}{l}\text { Root weight } \\
\text { response }\end{array}$ \\
\hline 1" x 6" & 24 & 793,440 & 634,752 & \multirow{2}{*}{ Increases } \\
\hline 3" x 6" & 12 & 396,720 & 317,376 & \multirow{2}{*}{238,032} \\
\hline 6" x 6" & 9 & 297,540 & 158,688 & \\
\hline 9" x 6" & 6 & 198,360 & & \\
\hline
\end{tabular}

*Adapted from Persons (1994) 
Table 3.3. Projected returns from sorting of roots to different morphotypes at farm level prior to selling under current $P$. quinquefolius cultivation system (no cultivars).

\begin{tabular}{|c|c|c|c|c|c|c|c|c|c|c|}
\hline \multirow[t]{2}{*}{$\begin{array}{l}\text { Root } \\
\text { types }\end{array}$} & \multirow{2}{*}{$\begin{array}{l}\text { proportion } \\
(\%) \text { of total } \\
\text { roots/acre* }\end{array}$} & \multirow[t]{2}{*}{$\begin{array}{l}\text { No. of roots } \\
\text { per acre }\end{array}$} & \multirow[t]{2}{*}{$\begin{array}{l}\text { Roots per } \\
\text { pound }\end{array}$} & \multirow[t]{2}{*}{$\begin{array}{l}\text { Pounds per } \\
\text { acre }\end{array}$} & \multicolumn{2}{|c|}{$\begin{array}{l}\text { Premium per } \\
\text { pound }(\$)^{* *}\end{array}$} & \multicolumn{2}{|c|}{$\begin{array}{l}\text { Gross returns per acre } \\
\text { (\$) after } 8 \text { years }\end{array}$} & \multicolumn{2}{|c|}{$\begin{array}{l}\text { Gross returns per acre } \\
(\$) \text { after } 8 \text { years }\end{array}$} \\
\hline & & & & & $5 \%$ & $30 \%$ & $5 \%$ & $30 \%$ & $5 \%$ & $30 \%$ \\
\hline ML & 15 & 35,705 & 34 & 1,037 & 63 & 78 & 65,352 & 80,911 & 65,352 & 80,911 \\
\hline BLB & 22 & 52,367 & 63 & 828 & 60 & 60 & 49,691 & 49,691 & 49,691 & 49,691 \\
\hline STK & 63 & 149,960 & 71 & 2,117 & 60 & 60 & 127,037 & 127,037 & 127,037 & 127,037 \\
\hline \multicolumn{7}{|c|}{ Gross returns from sorting (total of ML, BLB, STK) } & 242,079 & 257,639 & 242,079 & 257,639 \\
\hline \multicolumn{7}{|c|}{ Less sorting labor ${ }^{¥}$} & 1,000 & 1,000 & $3,112^{\text {井 }}$ & 3,112 \\
\hline \multicolumn{7}{|c|}{ Net return ${ }^{\Phi}$} & 241,079 & 256,639 & 238,967 & 254,527 \\
\hline MXD & & & & 3983 & 60 & 60 & 238,967 & 238,967 & 238,967 & 238,967 \\
\hline \multicolumn{7}{|c|}{ Value added return from sorting (over MXD) } & 2,112 & 17,672 & 0 & 15,560 \\
\hline
\end{tabular}

* Based on frequency distribution of morphotypes in our sample

** Price based on market price range for cultivated ginseng (\$30 - \$120) (Cheng and Mitchell 2009) with a 5\% or $30 \%$ premium mark up for ML morphotype based on its quality (high ginsenoside content). Price varies with age, production system, and market.

¥ Sorting labor based on cost for picking berries per acre (grower’s estimates)

${ }^{\phi}$ Returns only accounts for sorting costs. All other production costs up to harvesting are not included

\# Sorting labor cost to break even at $5 \%$ premium mark up for ML roots 
Table 3.4. Projected returns of cultivating select cultivar of $P$. quinquefolius.

\begin{tabular}{|c|c|c|c|c|c|c|c|c|c|}
\hline \multirow[t]{2}{*}{$\begin{array}{l}\text { Root } \\
\text { types }\end{array}$} & \multirow[t]{2}{*}{$\begin{array}{l}\text { Ave. dry wt } \\
\text { per root }(g)^{*}\end{array}$} & \multirow[t]{2}{*}{$\begin{array}{l}\text { No. of roots } \\
\text { per pound }\end{array}$} & \multirow[t]{2}{*}{$\begin{array}{l}\text { Pounds per } \\
\text { acre }^{\S}\end{array}$} & \multicolumn{2}{|c|}{$\begin{array}{l}\text { Price per pound } \\
(\$)^{* *}\end{array}$} & \multicolumn{2}{|c|}{$\begin{array}{l}\text { Gross returns per acre }(\$) \\
\text { after } 8 \text { years } \Phi\end{array}$} & \multicolumn{2}{|c|}{$\%$ return over $\mathrm{MXD}$} \\
\hline & & & & $5 \%$ & $30 \%$ & $5 \%$ & $30 \%$ & $5 \%$ & $30 \%$ \\
\hline ML & 13.19 & 34 & 6,916 & 63 & 78 & 435,708 & 539,410 & 82 & 126 \\
\hline BLB & 7.18 & 63 & 3,764 & 60 & 60 & 225,868 & 225,868 & -5 & -5 \\
\hline STK & 6.41 & 71 & 3,361 & 60 & 60 & 201,646 & 201,646 & -16 & -16 \\
\hline MXD & & & 3,983 & 60 & 60 & 238,967 & 238,967 & & \\
\hline
\end{tabular}

*Weight is for 8 year old root grown at 6" x 6" planting density

$\S$ Based on number of roots produced per acre $(238,032)$

**Price based on market price range for cultivated ginseng (\$30 - \$120) (Cheng and Mitchell 2009) with a 5\% or $30 \%$ premium mark up for ML morphotype based on its quality (high ginsenoside content). Price varies with age, production system, and market.

${ }^{\Phi}$ All other production costs up to harvesting are not included 


\section{CHAPTER 4}

Growth characteristics and ginsenosides production of in vitro tissues of American ginseng (Panax quinquefolius L.) 


\begin{abstract}
In vitro propagation of three root morphotypes (BLB, ML, STK; herein referred as lines) of Panax quinquefolius exhibited varied callus induction response and ginsenoside production on solidified MS medium supplemented with $1.0 \mathrm{mg} \mathrm{l}^{-1} 2,4-\mathrm{D}$ and $0.1 \mathrm{mg} \mathrm{l}^{-1}$ kinetin. Explants from ML line induced callus the fastest, were prolific in growth and accumulated more biomass compared to explants from other lines. Ginsenoside analysis of the stock roots and their respective callus tissues indicated that ML line had the highest ginsenosides content followed by BLB and STK lines. Comparisons between total ginsenoside content of stock roots and callus dry weight, and that between total ginsenoside contents of stock roots and callus tissues showed positive and highly significant correlations. Ginsenoside profiles of stock roots varied among lines. ML lines exhibited low Rg1/high Re profiles exclusively, whereas BLB and STK lines exhibited both low $\mathrm{Rg} 1 /$ high $\mathrm{Re}$ and high $\mathrm{Rg} 1 /$ low Re profiles. RAPD analysis of genomic DNA of stock roots showed polymorphism within and among lines, and when genetic diversity of stock roots and their total ginsenosides content were compared some relationship was revealed, however a link between a particular DNA band or banding patterns and specific ginsenosides profiles or abundance was not apparent. Genetic diversity, varied in vitro response, differences in ginsenosides profiles and abundance among ginseng lines presented in this study could be useful in progressing breeding efforts of ginseng and in vitro production of its bioactive components.
\end{abstract}




\section{Introduction}

Panax quinquefolius L., commonly referred to as American ginseng, is a perennial herb that is native to eastern deciduous woodlands of North America (Catling et al. 1994). For many years it has been cultivated in several regions in the USA and Canada for its highly valued root (Pritts 1995). American ginseng root is renowned for its calming properties, therefore used to reduce stress, lower blood pressure and boost the body's immunity (Vuksan et al. 2001). The pharmacological properties of ginseng are attributed to a group of secondary metabolites called ginsenosides (Attele et al. 1999). Besides of their use as therapeutic agents, ginsenosides are also marketed as dietary supplements and are often included as active ingredients in health foods and energy drinks (Shen et al. 2003; Qu et al. 2009).

For several years, the source of American ginseng root has been from the wild; however reports have indicated that its abundance in the wild is rapidly declining due to over collection, habitat degradation and fragmentation (Nantel et al. 1996; Robbins 1998). Efforts to cultivate ginseng are increasing lately as a response to increasing demand from rapidly expanding pharmaceutical and beverage industries, however field cultivation is labor intensive and time consuming due to the slow-growing nature of this plant (Proctor 1996). It takes 5-7 years from seedling to the final marketable root size, and during that time a lot of care must be given to plants since plant growth is exposed to various pathogens and pests (Proctor 1996). In addition, there are no improved cultivars of P. quinquefolius (Schluter and Punja 2002) even though several studies have documented that this plant is genetically diverse (Bai et al. 1996; Boehm et al. 1999; 
Schluter and Punja 2002; Cruse-Sanders and Hamrick 2004, Grubbs and Case 2004), shows considerable variation in its ginsenoside contents and profiles (Assinewe et al. 2003; Lim et al. 2005; Schlag and McIntosh 2006), and its root displays varied shapes which considerably influence its value and desirability among consumers (Roy et al. $2003 ; 2008)$.

To circumvent the problems associated with traditional cultivation, researchers have attempted to produce the bioactive components of ginseng using in vitro cultures (Wu and Zhong 1999). With in vitro culture, production is more controllable with regard to quality and quantity, since cell lines with desirable productivity can be selected $(\mathrm{Wu}$ and Zhong 1999). Since the first report on in vitro culture of $P$. quinquefolius by Jhang et al. (1974), there has been only few reports on tissue culture of $P$. quinquefolius compared to numerous publications on other species of genus Panax such as $P$. ginseng and $P$. notoginseng. The few reports on P. quinquefolius tissue culture have basically focused on optimizing in vitro conditions either for cell growth and ginsenoside production by suspension cultures or plantlet regeneration through somatic embryogenesis (Wang 1990; Mathur et al. 1994; Zhong et al. 1996; Tirajoh et al. 1998; Wang et al. 1999; Punja et al. 2004; Zhou and Brown 2005). Even though ginsenoside production in vitro has been successful for genus Panax, fluctuation of ginsenoside content is often reported (Wu and Zhong 1999). Variability of ginsenoside production in vitro has been attributed to use of different plant growth hormone combinations and concentrations (Zhong et al. 1996), however influence of genotype, which can be remarkable, has not been extensively studied in P. quinquefolius. 
It has been reported that explants from Azadirachta indica A. Juss (neem) plants with high and low azadirachtin-A (secondary metabolite) content displayed varied response to callus induction and subsequent accumulation of secondary metabolites in vitro (Kota et al. 2006). From a previous study on phytochemical content among different root morphotypes commonly encountered in ginseng (chapter 3), it was reported that roots of "man-like' (ML) morphotype contained high amount of ginsenoside content compared to those of "bulb or round" (BLB) and "straight or stick" (STK) morphotypes. In this study, the objectives were to: 1) evaluate how explants from the three ginseng morphotypes (hereafter referred to as ginseng lines) respond to callus induction and growth in vitro; 2) compare ginsenosides contents and profiles between stock plants and their subsequent callus tissues developed in vitro; and 3) assess genetic diversity among stock plants of the three ginseng lines.

\section{Materials and Methods}

Plant materials - Four year old roots of different ginseng lines were collected from a commercial ginseng farm in West Virginia and transplanted to raised beds of sandy loam soils at the experimental plot at West Virginia University Agronomy farm and the plants were left to grow for two years before they were used as explant sources for in vitro culturing. Upon harvesting roots for in vitro culture, leaf samples from donor plants were collected and frozen in liquid nitrogen then stored at $-80^{\circ} \mathrm{C}$ for later use in DNA extraction and subsequent genetic analysis using RAPD markers. Selected roots were rinsed with tap water, and half of each root was cut and set aside for ginsenoside analysis, and the other half was used for explants materials for in vitro studies. 
Explant preparation and tissue culture - Roots were surface sterilized by immersing in $70 \%$ ethanol for $1 \mathrm{~min}$, followed by soaking in $10 \%$ bleach with Tween® 20 (polyoxyethlenesorbitan monolaurate; PhytoTechnology Laboratories; Shawnee Mission, KS, USA) for 15 min with constant agitation, and finally rinsing three times in sterile distilled water. The culture medium was MS (Murashige and Skoog 1962) supplemented with $30 \mathrm{~g} \mathrm{l}^{-1}$ sucrose, $2 \mathrm{ml} \mathrm{l}^{-1} \mathrm{PPM}^{\mathrm{TM}}$ (Plant Preservation Mixture; Plant cell Technology, Washington, DC, USA), $1.0 \mathrm{mg} \mathrm{l}^{-1}$ 2, 4-D (Product no. D299; PhytoTechnology Laboratories; Shawnee Mission, KS, USA), $0.1 \mathrm{mg} \mathrm{l}^{-1}$ kinetin (Product no. K750; PhytoTechnology Laboratories; Shawnee Mission, KS, USA) and $8 \mathrm{~g} \mathrm{l}^{-1}$ agar (Product no. A111; PhytoTechnology Laboratories; Shawnee Mission, KS, USA). The pH of the medium was adjusted to 5.8 using either $1 \mathrm{~N} \mathrm{NaOH}$ or $\mathrm{HCl}$ prior to adding agar and autoclaving $\left(121^{\circ} \mathrm{C}\right.$ and $105 \mathrm{kPa}$ for $\left.20 \mathrm{~min}\right)$. The explants were excised from each root stock using a stainless steel cylindrical core borer (6 $\mathrm{mm}$ inside diameter), and were cut into small discs of equal sizes measuring $6 \mathrm{~mm}$ thick and $6 \mathrm{~mm}$ wide. The explants discs were transferred into $25 \times 150 \mathrm{~mm}$ borosilicate glass culture tubes containing $20 \mathrm{ml}$ of sterilized culture medium and capped with autoclavable Kim Kap® plastic caps (Kimble Glass, Inc; Toledo, OH, USA). Explants were cultured in the dark for 12 wks at a temperature of $24 \pm 1^{\circ} \mathrm{C}$ and were transferred to fresh medium every 4 wks. For each ginseng line there were six biological replicates with five explants in each replicate.

In vitro data collection and analysis - Callus formation data including average number of days it took explants in each line to induce callus, and percentages of explants with callus cells in each line at 14, 21, and 28 days were collected. Dry weight of callus tissue 
for each replicate explant disc of each biological root replicate was determined at the end of the experiment (12 wks) and averaged for each line. Growth rate of callus tissues for each line was computed as follows:

$$
\text { Growth rate }=\frac{(\text { Final }- \text { Initial }) \text { explant dry weight }}{\text { Initial explant dry weight }} \times 100
$$

Final dry weights of callus tissue among ginseng lines were analyzed using one-way analysis of variance in MINITAB release 13.20 (MINITAB, State College, PA, USA).

DNA extraction - Approximately $20 \mathrm{mg}$ of leaf material was placed in $1.5 \mathrm{ml}$ micro centrifuge tube and ground with a sterile disposable plastic pestle (Fisher Scientific, Pittsburgh, PA, USA). DNA extraction was done using a GenElute Plant Genomic DNA Mini Prep Kit (Catalog no. G2N70, Sigma-Aldrich, St. Louis, MO, USA) following the manufacturer's instructions. Quality of isolated genomic DNA was assessed by running $10 \mu \mathrm{l}$ of DNA template through $1.5 \%$ agarose gel in $0.5 \mathrm{x}$ TBE buffer (Tris-base, Boric acid, and EDTA). Gels were stained with ethidium bromide (EtBr), then visualized on electronic UV transilluminator (ULTRA-LŪM, Inc., Claremont, CA, USA) and digitally photographed with Canon Powershot G6 camera (Canon USA, Inc.). A single high molecular genomic DNA band devoid of a smear indicated intact high quality genomic DNA suitable for subsequent RAPD analysis. For each sample, isolated genomic DNA was quantified and diluted to $20 \mathrm{ng} / \mu \mathrm{l}$ with milliQ water and stored at $-20^{\circ} \mathrm{C}$ until when needed for RAPD analysis. 
RAPD analysis and DNA electrophoresis - Eight decamer primers that were successful for RAPD analysis in chapter 2 were used for PCR amplification of ginseng lines in this study. PCR amplifications were conducted using $\operatorname{HotStarTaq}^{\circledR}$ Master Mix Kit ( Catalog no. 203443, Qiagen ${ }^{\circledR}$, Germantown, MD, USA) with each $25 \mu 1$ reaction mixture containing: $12.5 \mu \mathrm{l}$ Hotstart mix, $11 \mu \mathrm{l}$ of RNase free $\mathrm{H}_{2} \mathrm{O}, 0.5 \mu \mathrm{l}$ of primer $(0.2 \mu \mathrm{M})$, and $1 \mu 1$ of DNA template $(\sim 20 \mathrm{ng})$. All amplifications were done on a single thermo cycler (GeneAmp ${ }^{\circledR}$ PCR System 9700, Applied Biosystems) with PCR thermocycling based on Schluter and Punja (2002) protocol with some modifications. The initial PCR cycle included heating the reaction mixture for $15 \mathrm{~min}$ at $94^{\circ} \mathrm{C}$ to activate the HotStarTaq DNA polymerase, as recommended by the manufacture, then $10 \mathrm{~min}$ at $36^{\circ} \mathrm{C}$, and $2 \mathrm{~min}$ at $72^{\circ} \mathrm{C}$. Subsequent 46 cycles included denaturation at $94^{\circ} \mathrm{C}$ for $30 \mathrm{~s}$, annealing at $36^{\circ} \mathrm{C}$ for $1 \mathrm{~min}$, and elongation at $72^{\circ} \mathrm{C}$ for $1 \mathrm{~min}$. In the final cycle primer elongation at $72^{\circ} \mathrm{C}$ was extended for $10 \mathrm{~min}$. Ten micro litres of amplified mix was loaded into $1.5 \%$ agarose gels alongside $1 \mathrm{~kb}$ DNA ladder (Promega, Madison, WI, USA) and separated electrophoretically in $0.5 \mathrm{x}$ TBE buffer at $105 \mathrm{~V}$ for $40 \mathrm{~min}$. Gels were stained with $\mathrm{EtBr}$, visualized with electronic UV transilluminator, and digitally photographed with Canon Powershot G6 camera (Canon USA, Inc.).

Data analysis - RAPD amplification profiles of each primer for all ginseng lines were scored by visual observation of gel pictures. The DNA bands were scored as present (1) or absent (0) at each position. Monomorphic bands across all samples and bands below $250 \mathrm{bp}$ or above $3000 \mathrm{bp}$ were omitted from further analysis. From the RAPD data, Nei's (1978) unbiased distances matrix was calculated to estimate the pair-wise distances 
among individual roots and among ginseng lines. A cluster analysis was done using Unweighted pair group method (UPGMA) based on Nei's coefficient to generate dendrograms displaying genetic relationship within and among ginseng lines using TFPGA software program (Miller 1998) with 1000 bootstraps.

Ginsenoside extraction - Ginsenosides were extracted following the method of Schlag and McIntosh (2006) with slight modifications. Fifty milligrams of root or callus powder was transferred into $10 \mathrm{ml}$ glass vials with vented cap, and $5 \mathrm{ml}$ of $80 \% \mathrm{MeOH}$ was added, briefly mixed by vortexing and the mixture was incubated in a water bath at $70^{\circ} \mathrm{C}$ with constant sonication for $1 \mathrm{hr}$. Thereafter, the vials were centrifuged for 5 minutes at 2000 rcf using a Mistral 3000i centrifuge (Curtin Matheson Scientific, Inc., Houston, TX, USA), and the supernatant was transferred into clean tubes. The residues were reextracted once and the supernatants from both extractions were combined and reduced to dryness under a stream of $\mathrm{N}_{2}$ at $38^{\circ} \mathrm{C}$, and then re-suspended in $2 \mathrm{ml}$ solvent of 20:20:60 (MeOH: $\mathrm{ACN}: \mathrm{H}_{2} \mathrm{O}$ ). The concentrated extracts were filtered through a $0.45 \mu \mathrm{m}$ membrane filters into clean $2 \mathrm{ml}$ polypropylene tubes (Spin- ${ }^{\circledR} 8162$; Corning Inc. Corning, NY, USA) and stored at $4{ }^{\circ} \mathrm{C}$ until HPLC analysis $(<24 \mathrm{hrs})$.

HPLC analysis - The HPLC system used was a Waters model 626 separation module (Milford, MA, USA) equipped with a Tunable Absorbance Detector (Waters 486), an auto sampler (Waters 717 plus), and Waters Empower software. The separation was carried out in Atlantis ${ }^{\circ} \mathrm{T} 3$ column $(5 \mu \mathrm{m}, 250$ x $4.6 \mathrm{~mm})$ with Atlantis® T3 guard cartridge $(5 \mu \mathrm{m}, 4.6 \times 20 \mathrm{~mm})$, Waters, Inc. (Milford, MA, USA). The UV spectra for 
ginsenosides were recorded at $203 \mathrm{~nm}$. For HPLC analysis, a $20 \mu 1$ sample or standard was injected into the column and eluted at room temperature at a constant flow rate of 1.2 $\mathrm{ml} / \mathrm{min}$. The mobile phase used for separation consisted of solvent A (100\% water), and solvent B (100\% acetonitrile). A mobile phase gradient was based on that of Wang et al (2006) with slight modifications to ensure good separation of peaks with our HPLC system: From $0-20 \mathrm{~min}, 80 \% \mathrm{~A}$ and $20 \% \mathrm{~B}$; from $20-29 \mathrm{~min}, 74 \% \mathrm{~A}$ and $26 \% \mathrm{~B}$; from $29-43 \mathrm{~min}, 66 \% \mathrm{~A}$ and $34 \% \mathrm{~B}$; from $43-47 \mathrm{~min}, 64 \% \mathrm{~A}$ and $36 \% \mathrm{~B}$; from 47 $54 \min , 57 \% \mathrm{~A}$ and $43 \% \mathrm{~B}$; from $54-59 \mathrm{~min}, 5 \% \mathrm{~A}$ and $95 \% \mathrm{~B}$.

A ginsenoside standards kit containing $5 \mathrm{mg}$ each of $\mathrm{Rb} 1, \mathrm{Rb} 2, \mathrm{Rc}, \mathrm{Rd}, \mathrm{Re}$, and Rg1 (purity > 99\%) was purchased from Indofine Chemical Company, Inc. (Catalog no. 020119S, Hillsborough, NJ, USA). Serial concentration dilutions were made for individual standards and were injected into HPLC, and corresponding peak areas for each standard concentration were used to make standard curves (peak areas were averages of three runs per standard concentration). All six ginsenoside standards were combined and $20 \mu l$ of the mixture was injected to obtain retention times for respective ginsenosides. The standards solutions were prepared using the same solvent as for the samples. Individual ginsenosides in each sample were identified by their retention times corresponding to those obtained from chromatogram of mixed standards. In cases where peaks of some ginsenosides would not be clearly identified, samples were spiked with known standard concentration to identify the peaks and quantification of ginsenosides in the sample was calculated by subtracting peak areas of spiked standards alone from total peak area (sample and standard spike). Samples were injected thrice per day (to check 
for intraday variability) with samples ordered randomly in each run, and random samples would be injected on different days (to check for interday variability). Intra and interday variability (RSD) of ginsenoside content was $<5 \%$. Individual ginsenoside content in each sample were calculated using their respective standard curves $\left(r^{2}>0.99\right)$. The sum of six individual ginsenosides (hereafter referred to as total ginsenosides) across ginseng lines was analyzed using one-way analysis of variance in MINITAB release 13.20 (MINITAB, State College, PA, USA).

\section{Results and Discussion}

Callus induction and growth - Callus induction was noted at the cut ends of explants at varied times for ginseng lines, however all explants developed callus tissues within 4 wks, which is in agreement with what was reported previously on tissue culture of $P$. quinquefolius (Punja et al. 2004). All callus tissues were light brown in color, soft and friable. On average explants of ML line developed callus tissues in 14 days, whereas those of BLB and STK lines took on average 19 and 23 days respectively (Figure 4.1). Number of explants with callus tissues varied among ginseng lines. Between day 7 and $14,52 \%$ of explants in ML had developed callus tissues, compared to $17 \%$ of explants in BLB line, and zero explants in STK line. By day 21, 38\% of explants for STK line had developed callus whereas $83 \%$ and $100 \%$ of explants from BLB and ML lines respectively had callus at this time (Figure 4.2). Variability in callus induction and growth among explants from different lines has been reported in P. quinquefolius (Punja et al. 2004). Given that in this study all explants were cultured in the same medium 
under controlled conditions, it is suggestive that such variability in callus induction and growth would be due to genetic variations observed among source plants (Figure 4.6).

Kota et al (2006) reported that in Azadirachta indica A. Juss, explants from high azadirachtin-A (a secondary metabolite) stock plants exhibited a better callus induction response and growth than those from low azadirachtin-A stock plants. Similar observation was made in this study. Explants of ML lines (which had high total ginsenosides content) induced callus in a short time and grew faster than those from other lines (Figure 4.1; Table 4.1). Overall, dry weights of callus tissues at the end of the culture period were highest in ML lines, followed by BLB lines, and STK lines were the lowest (Figure 4.3). Callus dry weight was positively correlated with total ginsenosides content of stock plants $(\rho=0.796)$ (Table 4.2). When values of total ginsenosides contents of stock roots were plotted against callus dry weight and a linear regression equation was fitted into the data (Figure 4.4), the following equation resulted:

$$
\mathrm{CDW}=3.02 \mathrm{GCSR}+17.45\left(\operatorname{Adj} \mathrm{r}^{2}=0.6102\right)
$$

Where:

$$
\begin{aligned}
& \mathrm{CDW}=\text { callus dry weight }(\mathrm{mg}) \text {, and } \\
& \mathrm{GCSR}=\text { total ginsenosides content of stock root }(\mathrm{mg} / \mathrm{g})
\end{aligned}
$$

Analysis of regression showed that the equation (Eq.1) was significant $(P=0.001)$, implying that up to $61.02 \%$ of the variability in callus growth is accounted by ginsenosides content of stock plants. 
Ginsenosides analysis - Despite being of the same age the total ginsenosides content of stock roots ranged from 14.31 to $41.14 \mathrm{mg} / \mathrm{g}$ (Table 4.3). Overall mean total ginsenosides content was lowest in STK line $(23.16 \mathrm{mg} / \mathrm{g})$, whereas ML line had the highest $(35.08 \mathrm{mg} / \mathrm{g}$ ) total ginsenosides content (Table 4.4). Comparison of mean total ginsenosides among lines showed significant differences; these differences were between ML lines and BLB or STK roots, but not between BLB and STK lines (Table 4.4). Ginsenosides Rg1, Re, and Rb1 varied significantly among ginseng lines, however variation of ginsenosides $\mathrm{Rc}, \mathrm{Rb} 2$, and $\mathrm{Rd}$ among lines was not significant (Table 4.4). $\mathrm{Rb} 1$ was the most abundant ginsenoside on all lines (Table 4.3 and 4.4), which is in line with other reports that have quantified ginsenosides in P. quinquefolius (Assinewe et al 2003, Lim et al 2005, Schlag and McIntosh 2006). Rg1 and Re were inversely related among all lines. ML lines exhibited low Rg1/high Re profile, whereas BLB and STK lines exhibited both low $\mathrm{Rg} 1 /$ high Re and high $\mathrm{Rg} 1 /$ low Re profiles, but the proportion of roots with high $\mathrm{Rg} 1 /$ low Re profile were high in both BLB and STK lines (83\% and 66\% respectively) (Table 4.3 and 4.4).

Callus tissues contained less total ginsenosides content than the source roots (Table 4.4), which was expected and is in line with what has been reported before for this species (Wang et al. 1999). Their $\operatorname{Rg} 1 / \operatorname{Re}$ ginsenoside profiles were similar to those of their stock roots, and as in stock roots, $\mathrm{Rb} 1$ was the most abundant ginsenoside in callus tissues. However, the least abundant ginsenoside in callus tissues was Rd, whereas in stock roots it was ginsenoside $\mathrm{Rb} 2$. Overall total ginsenosides content was high in callus tissues of ML line followed by those of BLB line and calli of STK line had the least 
amount of total ginsenosides. The difference in amount of total ginsenosides between callus tissues of BLB and ML lines was not significant; however between STK line and ML or BLB lines was significant (Table 4.4). Total ginsenosides content of callus tissues correlated positively $(\rho=0.828)$ with total ginsenosides content of stock plants (Table 4.2). A linear regression analysis of total ginsenosides content of callus tissue with that of stock roots (Figure 4.5), gave the following equation:

$$
\mathrm{GCCT}=0175 \mathrm{GCSR}+7.75\left(\operatorname{Adj~} \mathrm{r}^{2}=0.6665\right)
$$

Where:

GCCT $=$ total ginsenosides content of callus tissue $(\mathrm{mg} / \mathrm{g})$, and GCSR $=$ total ginsenosides content of stock root $(\mathrm{mg} / \mathrm{g})$ Analysis of regression showed that the equation (Eq. 2$)$ was significant $(P=0.001)$, implying that ginsenosides content of stock roots (GCSR) accounts up to $66.65 \%$ of the variability in ginsenosides content of callus tissues (GCCT).

Even though many studies have reported biomass growth and saponin production of in vitro tissues of ginseng, few have addressed the correlation between callus biomass and its ginsenosides content. In this study, the correlation between callus dry weight and total ginsenosides content was determined, and was found to be positive and highly significant $(\rho=0.915)$ (Table 4.2). In Panax ginseng, such correlation was found to be negative (Wu and Zhong 1999), and in Panax notoginseng there was no correlation found (Zhang and Zhong 1997). The variability in correlation of in vitro cultures and their bioactive components in genus Panax can be attributable to factors such as genetic 
differences among the species, differences in their ginsenosides profiles, and variation of their growth kinetics and nutritional requirements in vitro (Hon et al. 2003; Mathur et al. 2003; Hong et al. 2005).

RAPD analysis - Out of the eight primers used, only six produced good reproducible and polymorphic bands. A total of 37 fragments were generated using the six primers, the number of bands per primer ranged from 5 (OPH-04) to $9(\mathrm{OPH}-09)$ with an average of 6.16 bands per primer. The percentage polymorphic bands ranged from $60 \%$ (UBC81) to $100 \%$ (OPD-05, OPH-05, and OPO-09) with a mean of $89.18 \%$ (Table 4.5). The smallest genetic distance was between ML-2 and BLB-6; ML-3 and STK-4; BLB-1 and BLB-2; and BLB-1 and BLB-3, whereas the largest genetic distance was between ML1and STK-6 (Table 4.6). UPGMA cluster analysis of individual plants showed a considerable amount of genetic variation within ginseng lines (Figure 4.6). One plant from ML line clustered separate from all other plants, and three plants from ML line clustered separately from the rest of the plants and were supported by a high bootstrap value. However, two plants from ML line clustered with plants of either BLB or STK lines. There was no distinct separation between plants of BLB and STK lines; they clustered more often with each other. When plants from each line were pooled such that genetic relationship would be compared at line level, BLB and STK lines clustered together and ML line clustered separately (Figure 4.7). Genetic dissimilarity among lines was; $11.15 \%$ between ML and BLB lines, $12.60 \%$ between ML and STK lines, and 2.60\% between BLB and STK lines (Table 4.7). 
When genetic diversity (Figure 4.6) was compared to variations in ginsenosides content of stock plants (Table 4.3) interesting observations were made. The plant with the highest amount of total ginsenosides (ML-1) clustered separately from all other plants. Similarly, the plant with the lowest total ginsenosides (STK-6) was an "outgroup" of its cluster. Also plants with the next highest amount of total ginsenosides (ML-4 and ML-6) clustered together. However, other plants that clustered together, meaning they were closely related genetically, had different ginsenoside profiles and total ginsenosides content. For example ML-2 and BLB-6 clustered together, but their Rg1/Re profiles were opposite, and had a 28\% difference in their total ginsenosides content. Likewise, ML-3 and STK-4 clustered together even though they had $42 \%$ difference in their total ginsenosides content and their $\operatorname{Rg} 1 / \operatorname{Re}$ profiles were opposite. Furthermore, BLB-1, BLB-2, and BLB-3 clustered together even though their $\operatorname{Rg} 1 / \operatorname{Re}$ profiles were different. BLB-1 and BLB-2 showed high Rg1/low Re profile whereas BLB-3 had low Rg1/high Re profile, however their total ginsenosides content were close $(26.09,25.32$, and 28.41 $\mathrm{mg} / \mathrm{g})$.

Examining of the banding patterns within and among lines did not reveal any unique DNA band or banding pattern that would be associated to a particular ginsenosides profile or that would explain abundance of ginsenosides on one plant over the other or one ginseng line over the other. Linkage of a particular DNA band or banding patterns to a given ginsenosides profile can possibly be attained with use of more DNA markers on a bigger sample size combined with ginsenoside analysis. 


\section{Conclusions}

This study has demonstrated that root morphotypes of $P$. quinquefolius have different callus induction response, callus growth, and ginsenoside production in vitro. Although it has been reported previously that proliferation of in vitro tissues and their subsequent ginsenoside production is influenced by plant growth regulators and chemical composition of the medium, through this study it has also been demonstrated that initial total ginsenosides content of explant donor plants have a significant influence on callus induction, growth, and resulting ginsenosides production in $P$. quinquefolius. Also data from this study suggests that there are genetic differences between root morphotypes of P. quinquefolius and that their ginsenoside profiles are variable. Although a specific link between a particular DNA band and a given ginsenosides profile or abundance was not found in this study, this foundational research will need to be expanded to identify DNA banding patterns that will categorize plants with desired profiles and ginsenoside content and therefore can be targeted for breeding of ginseng cultivars or to produce its bioactive components in vitro. 


\section{Literature Cited}

Assinewe, V. A., B. R. Baum, D. Gagnon, J. T. Arnason. 2003. Phytochemistry of wild populations of Panax quinquefolius L. (North American ginseng). Journal of Agricultural and Food Chemistry 51: 4549-4553.

Attele, A. S., J. A. Wu, C. S. Yuan. 1999. Ginseng pharmacology: multiple constituents and multiple actions. Biochememical Pharmacology 58: 1685-1693.

Bai, D., J. Brandle, and R. Reeleder. 1997. Genetic diversity in North American ginseng (Panax quinquefolius L.) grown in Ontario detected by RAPD analysis. Genome 40: 11-115.

Boehm, C. L., H. C. Harrison, G. Jung, and J. Nienhuis. 1999. Organization of American and Asian ginseng germplasm using randomly amplified polymorphic DNA (RAPD) markers. Journal American Society Horticulture Science 124: 252-256.

Catling, P. M., E. Haber, and E. Small. 1994. Poorly known economic plants of Canada: ginseng_Panax quinquefolius L. Canadian Botanical Association Bulletin 27: $70-72$.

Cruse-Sanders, J.M., and J.L. Hamrick. 2004. Genetic diversity in harvested and protected populations of wild American ginseng, Panax quinquefolius L. (Araliaceae). American Journal of Botany 91 (4): 540-548.

Grubbs, J.H., and M.A. Case. 2004. Allozyme variation in American ginseng (Panax quinquefolius L.): Variation, breeding system, and implications for current conservation practice. Conservation genetics 5: 13-23. 
Hon, C. C., C. Chow, F. Y. Zeng, and C. C. Leung. 2003. Genetic authentication of ginseng and other traditional medicinal Chinese plants. Acta Pharmacologica Sinica 24 (9): 841-846.

Hong, D. Y. Q., A. J. Lau, C. L. Yeo, X. K. Liu, C. R. Yang, H. L. Koh, and Y. Hong. 2005. Genetic diversity and variation of saponin contents in Panax notoginseng roots from a single farm. Journal of Agricultural and Food Chemistry 53: 84608467.

Jhang, J. J. A., E. J. Staba, and J. Y. Kim. 1974. American and Korean ginseng tissue cultures: Growth, chemical analysis, and plantlet production. In vitro cell and Developmental Biology-Plant 9: 253-259.

Kota, S., N. D. Raghupati Rao, and P. Chary. 2006. In vitro response of select regions of Azadirachta indica A. Juss (Meliaceae) as elucidated by biochemical and molecular variations. Current Science 9 (6): 770-776.

Lim, W., K. W. Mudge, and F. Vermeylen. 2005. Effects of population, age, and cultivation methods on ginsenoside content of wild American ginseng (Panax quinquefolium). Journal of Agricultural and Food Chemistry 53: 8498-8505.

Mathur, A., Y. N. Shukla, M. Pal, P. S. Ahuja, and G. C. Uniyal. 1994. Saponin production in callus and cell and cell suspension cultures of Panax quinquefolium. Phytochemistry 35: 1221-1225.

Mathur, A., A. K. Mathur, R. S. Sangwan, A. Gangwar, and G. C. Uniyal. 2003. Differential morphogenetic response, ginsenoside metabolism and RADP patterns of three Panax species. Genetic Resources and Crop Evolution 50: 245-252. 
Miller, M.P. 1998. TFPGA: A Windows ${ }^{\circledR}$ program for the analysis of allozyme and molecular population genetic data. Department of Biological Sciences, Northern Arizona University, Flagstaff, Arizona.

Murashige, T., and F. A. Skoog. 1962. A revised medium for rapid growth and bioassays with tobacco tissue cultures. Plant Physiology 15: 473-497.

Nantel, P., D. Gagnon, and A. Nault. 1996. Population Viability Analysis of American Ginseng and Wild Leek Harvested in Stochastic Environments. Conservation Biology 10 (2): 608- 621.

Nei, M. 1978. Estimation of average heterozygosity and genetic distance from a small number of individuals. Genetics 89: 583-590.

Pritts, K. D. 1995. How to find, grow, and use America's forest gold. Stackpole Books. Mechanicsburg, Pennsylvania, USA.

Proctor J. T. A. and W. G. Bailey. 1987. Ginseng: Industry, botany, and culture. Horticulture Reviews 9: 187-236.

Proctor, J. T. A. 1996. Ginseng: Old crop, new directions. In: Janick J (ed) Progress in new crops. ASHS Press, Arlington, Virginia, USA.

Punja, Z. K., M. Feeney, C. Schluter, and T. Tautorus. 2004. Multiplication and germination of somatic embryos of American ginseng derived from suspension cultures and biochemical and molecular analyses of plantlets. In vitro cell and Developmental Biology-Plant 40: 329-338.

Qu, C., Y. Bai, X. Jin, Y. Wang, K. Zhang, J. You, and H. Zhang. 2009. Study of ginsenosides in different parts and ages of Panax quinquefolius L. Food Chemistry 115: 340-346. 
Robbins, C. S. 1998. American ginseng: The root of North America's medicinal herb trade. TRAFFIC North America Report. Number B347, Washington, D.C. USA.

Roy, R. C., R. Grohs, and R. D. Reeleder. 2003. A method for the classification by shape of dried roots of ginseng (Panax quinquefolius L.). Canadian Journal of Plant Science 83: 955-958.

Roy, R. C., B. R. Ball Coelho, R. D. Reeleder, A. J. Bruin, R. Grohs, P. H. White, and B. B. Capell. 2008. Effect of planting bed shape, mulch and soil density on root yield and shape in North American ginseng (Panax quinquefolius L.). Canadian Journal of Plant Science 88 (5): 937-949.

Shen, X. H., Y. P. Ren, and Y. Chen. 2003. Detection of ginsenoside in health foods by HPLC. Chinese Journal of Health Laboratory Technology 5: 600-601.

Schlag, E. M. and M. S. McIntosh. 2006. Ginsenoside content and variation among and within American ginseng (Panax quinquefolius L.) populations. Phytochemistry 67: 1510-1519.

Schluter, C., and Z.K. Punja. 2002. Genetic diversity among natural and cultivated field populations and seed lots of American ginseng (Panax quinquefolius L.) in Canada. International Journal of Plant Science 163: 427-439.

Tirajoh, A., T. S. Kyung and Z. K. Punja. 1998. Somatic Embryogenesis and Plantlet Regeneration in American Ginseng (Panax quinquefolium L.). In vitro cell and Developmental Biology-Plant 34: 203-211.

Wang, A. 1990. Callus induction and plantlet regeneration of American ginseng. HortScience 25 (5): 571-572. 
Wang. X., J. T. A. Proctor, Y. Kakuda, S. KrishnaRaj, P. K. Saxena. 1999. Ginsenosides in American Ginseng: Comparison of in vitro Derived and Field-Grown Plant Tissues. Journal of Herbs, Spices \& Medicinal Plants 6 (3): 1-10.

Wang, C. Z., J. A. Wu, E. McEntee, and C. S. Yuan. 2006. Saponins composition in American ginseng leaf and berry assayed by high performance liquid chromatography. Journal of Agricultural and Food Chemistry 54: 2261-2266.

Wu, J., and J.-J. Zhong. 1999. Production of ginseng and its bioactive compounds in plant cell culture: current technological and applied aspects. Journal of Biotechnology 68: 89-99.

Vuksan, V., J. L. Sievenpiper, V. Y. Y. Koo, T. Francis, U. Beljan-Zdravkovic, Z. Xu, and E. Vidgen. 2001. American ginseng (Panax quinquefolius L.) reduces postprandial glycemia in non diabetic subjects and subjects with type II diabetes mellitus. Archives of Internal Medicine 160: 1009-1013.

Zhang, Y. H., and J. -J. Zhong. 1997. Hyperproduction of ginseng saponins and polysaccharide by high density cultivation of Panax notoginseng. Enzyme and Microbial Technology 21: 59-63.

Zhong, J. -J., Y. Bai, and S. -J. Wang. 1996. Effect of plant growth regulators on cell growth and ginsenoside saponin production by suspension cultures of Panax quinquefolium. Journal of Biotechnology 45: 227-234.

Zhou, S., and D. C. W. Brown. 2005. High efficiency plant production of North American ginseng via somatic embryogenesis from cotyledon explants. Plant Cell Reports 25(3):166-173. 


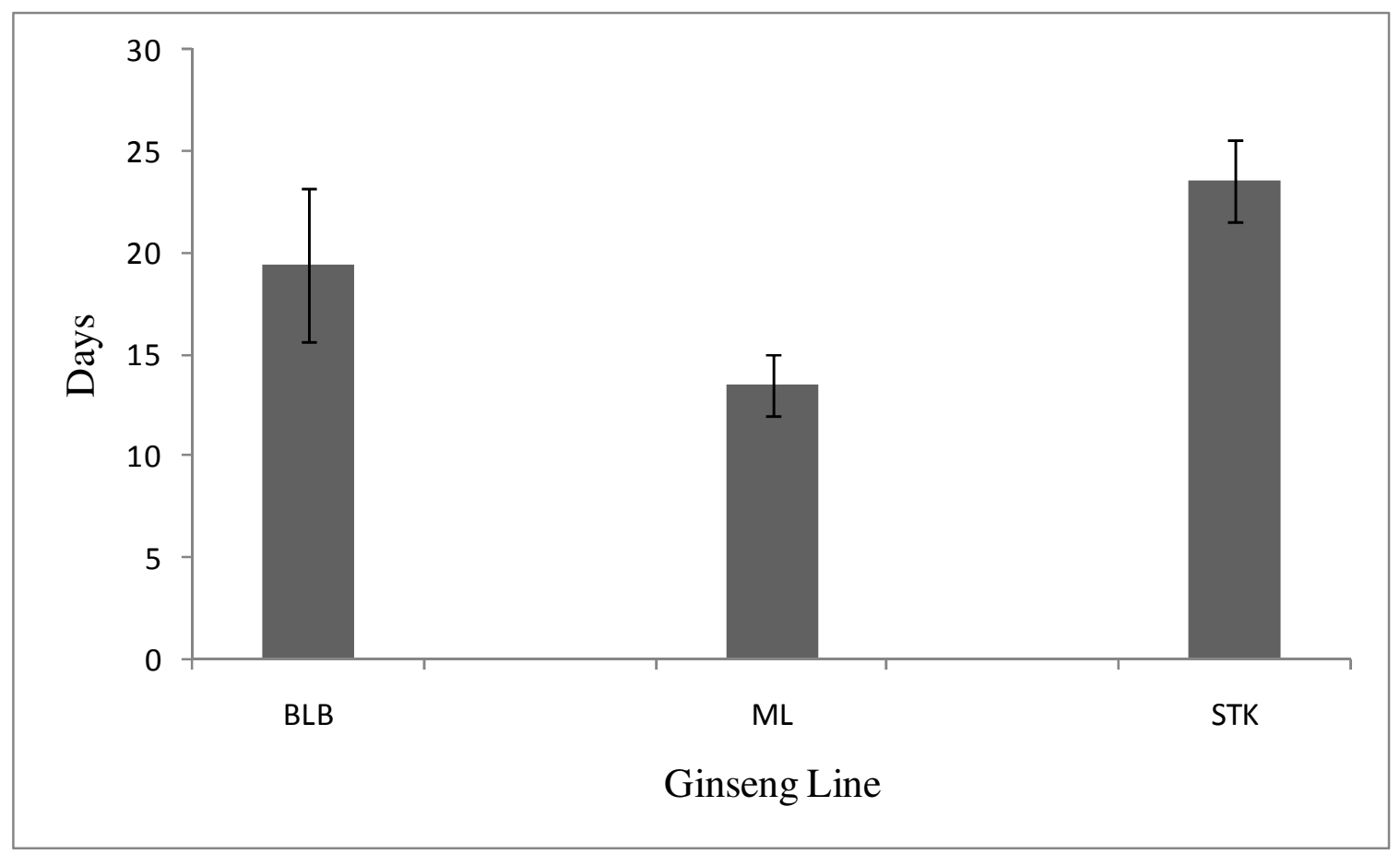

Figure 4.1. Callus induction time of root explants from different ginseng lines cultured in the dark in MS medium with $1.0 \mathrm{mg}^{-1} 2$, 4-D and $0.1 \mathrm{mg} \mathrm{l}^{-1}$ Kinetin. Each value is a mean of 6 biological replicates with 5 explants per replicate. Vertical lines are standard deviations of means. 


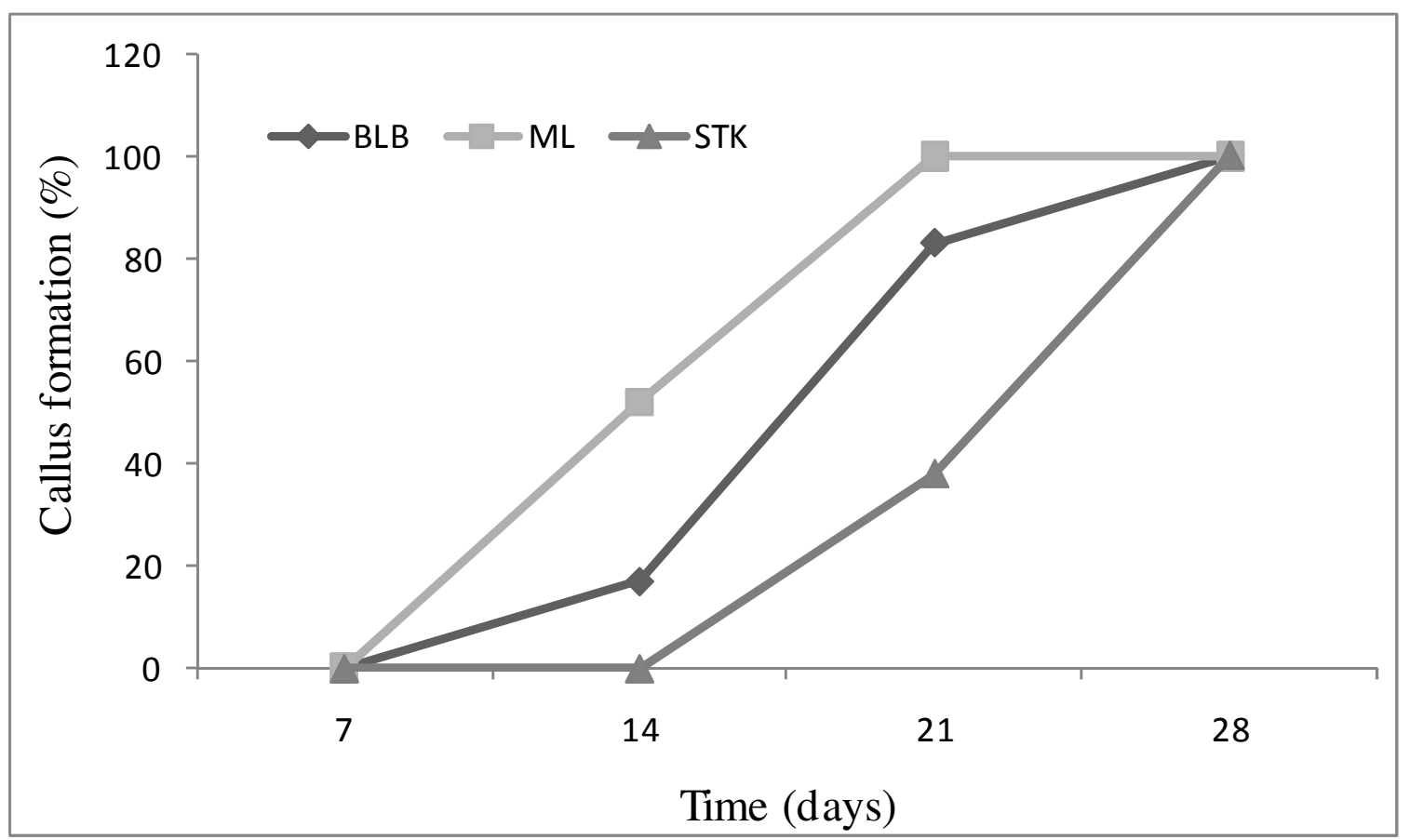

Figure 4.2. Frequency of callus formation on root explants of three American ginseng lines cultured in MS medium with $1.0 \mathrm{mg} \mathrm{l}^{-1} 2,4-\mathrm{D}$ and $0.1 \mathrm{mg} \mathrm{l}^{-1}$ kinetin. Data based on observation of 6 biological replicates with 5 explants per replicate for each ginseng line. 


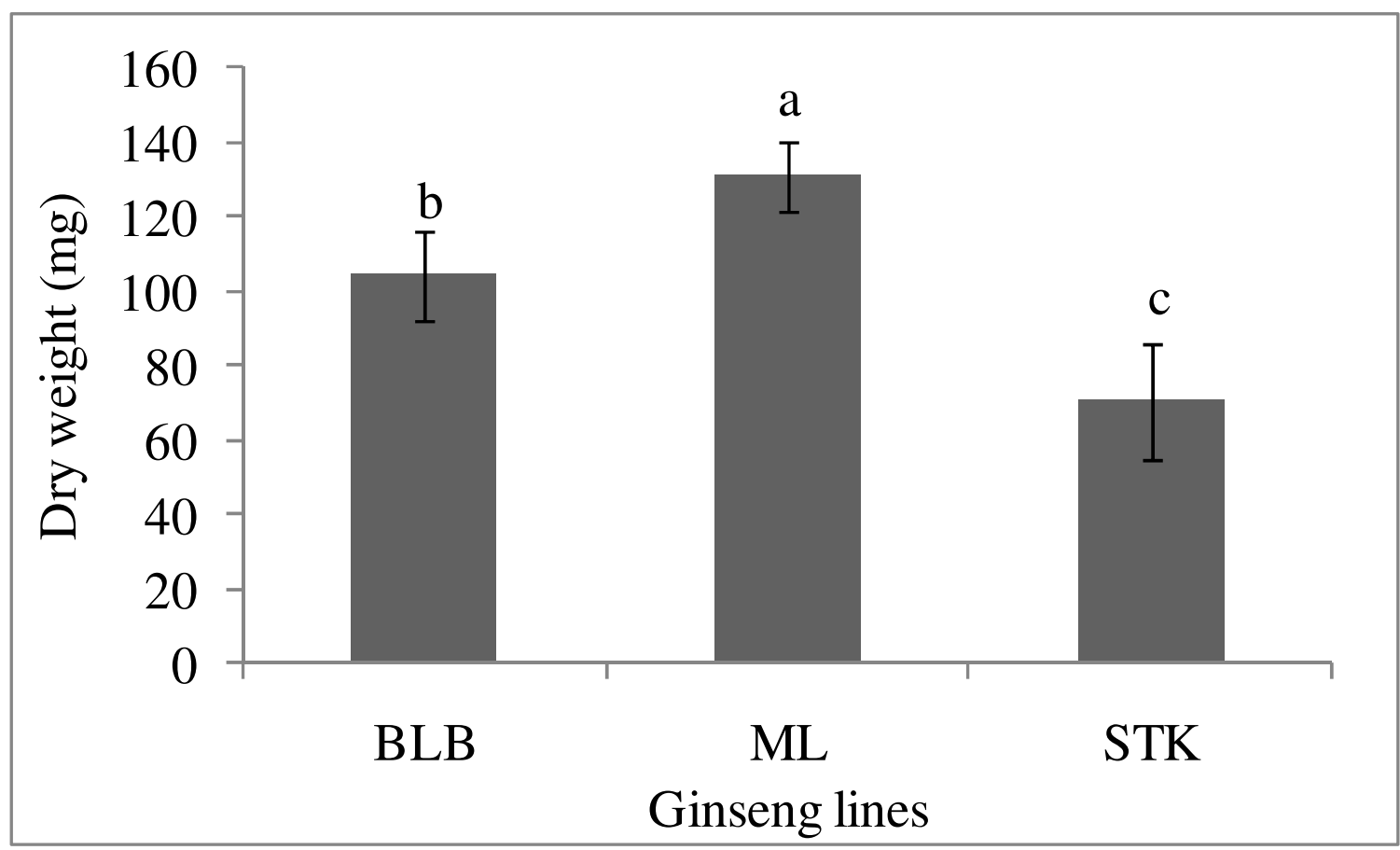

Figure 4.3. Callus growth on root explants of different ginseng lines cultured in the dark for 12 wks in MS medium with $1.0 \mathrm{mg} \mathrm{l}^{-1} 2,4-\mathrm{D}$ and $0.1 \mathrm{mg} \mathrm{l}^{-1}$ kinetin. Means are for 6 biological replicates with 5 explants per replicate for each ginseng line. Vertical lines are standard deviations of means. Different letters on each bar indicate means are significantly different at $\alpha=0.05$, Tukey's test. 


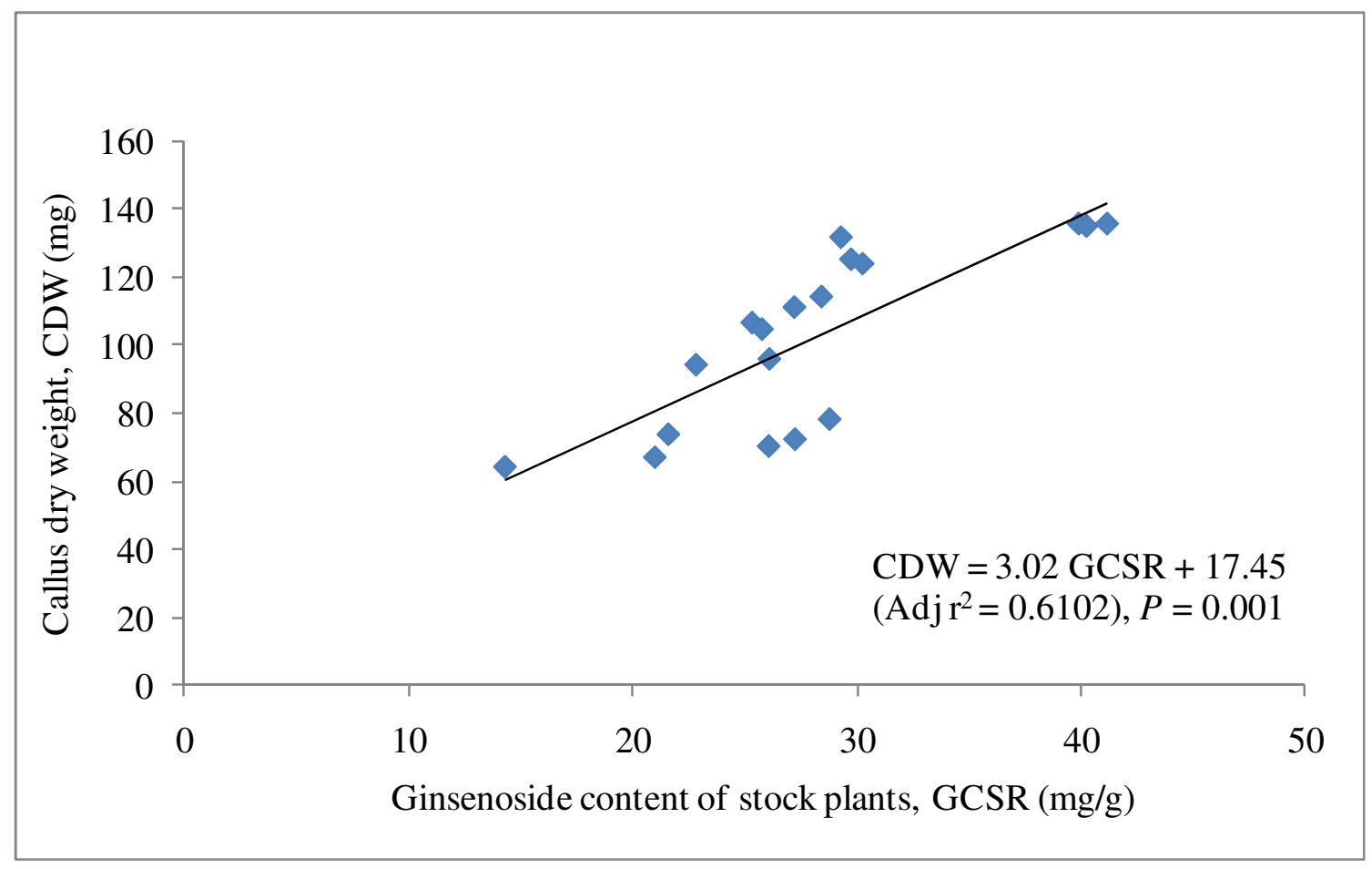

Figure 4.4. Relationship between total ginsenosides of stock plants and subsequent dry weight of their callus tissue after 12 wks of culture in the dark in MS medium with 1.0 $\mathrm{mg} \mathrm{l}^{-1} 2,4-\mathrm{D}$ and $0.1 \mathrm{mg} \mathrm{l}^{-1}$ Kinetin. The extreme values are not leverage points; their DFIT values did not exceed $2 \sqrt{ }[(k+1) / n]$, where $k$ is the number of predictors and $n$ the number of data points (Minitab Reference Manual, MINITAB). 


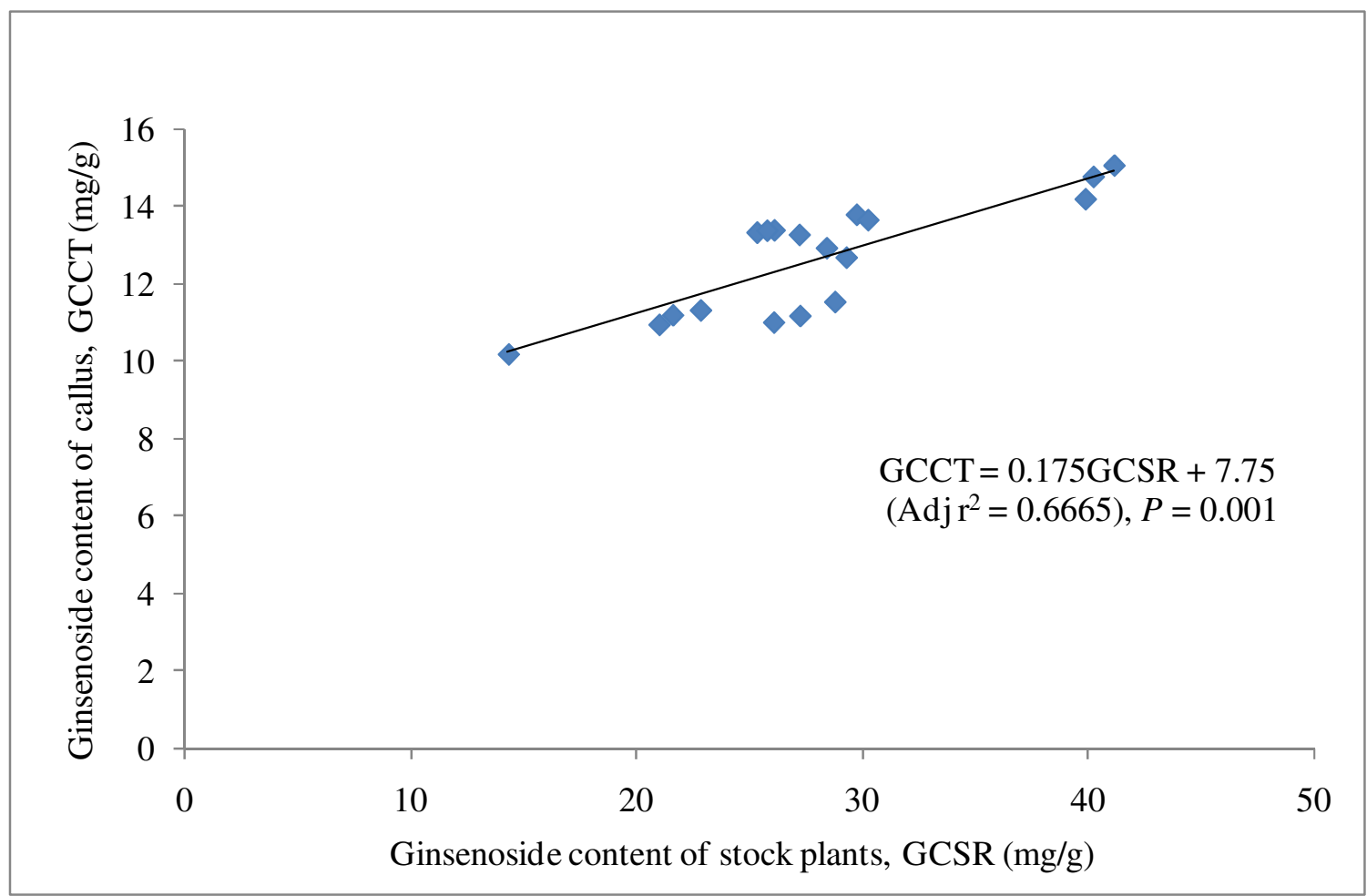

Figure 4.5. Relationship between total ginsenosides of stock roots and that of their callus tissue cultured for $12 \mathrm{wks}$ in the dark in MS medium with $1.0 \mathrm{mg} \mathrm{l}^{-1} 2,4-\mathrm{D}$ and $0.1 \mathrm{mg} \mathrm{l}^{-1}$ Kinetin. The extreme values are not leverage points; their DFIT values did not exceed $2 \sqrt{ }[(k+1) / n]$, where $k$ is the number of predictors and $n$ the number of data points (Minitab Reference Manual, MINITAB). 

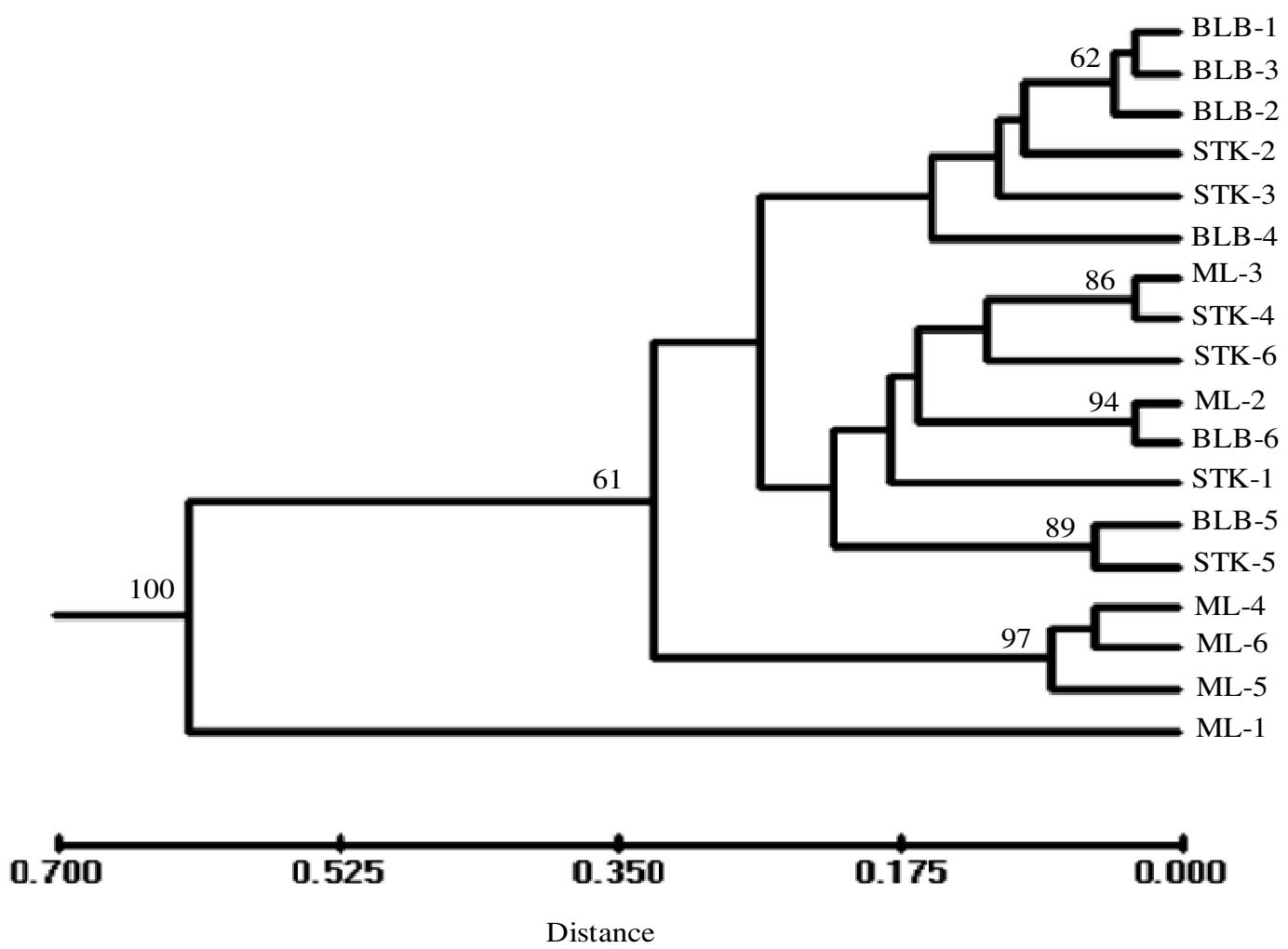

Figure 4.6. UPGMA based distance relationship between individual plants of $P$. quinquefolius with different root morphotypes (ginseng lines) based on Nei's (1978) unbiased distance of their molecular data from RAPD analysis with 1000 bootstraps. BLB = "bulb or round"; ML = "man-like"; STK = "straight or stick" morphotypes. Only bootstraps values greater than $60 \%$ are indicated. 


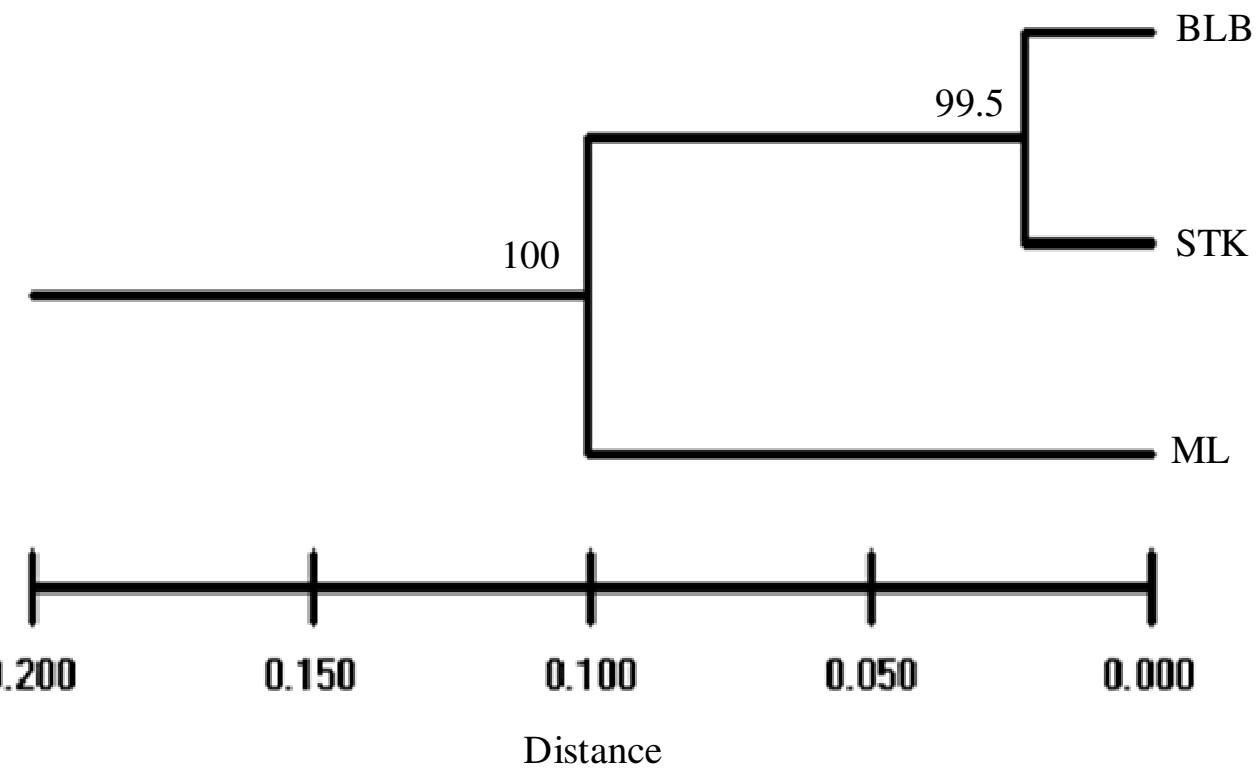

Figure 4.7. UPGMA based distance relationship between three root morphotypes (ginseng lines) of $P$. quinquefolius based on Nei's (1978) unbiased distance of their molecular data from RAPD analysis with 1000 bootstraps. BLB = "bulb or round"; ML = "man-like"; STK = "straight or stick" morphotypes. 
Table 4.1. Rate of callus growth of different ginseng lines cultured for $12 \mathrm{wks}$ in the dark in MS medium with $1.0 \mathrm{mg} \mathrm{l}^{-1} 2,4-\mathrm{D}$ and $0.1 \mathrm{mg} \mathrm{l}^{-1}$ kinetin.

\begin{tabular}{l|c}
\hline Ginseng line & Growth rate, \%* \\
\hline ML & 395 \\
STK & 168 \\
BLB & 294 \\
\hline
\end{tabular}

$*$ Growth rate $=\underline{(\text { Final }- \text { Initial }) \text { explant dry weight }} \times 100$

Initial explant dry weight 
Table 4.2. Pearson correlation among callus weight, total ginsenosides content of callus and stock roots. All correlations were highly significant $(P=0.001)$.

Stock root ginsenosides

content $(\mathrm{mg} / \mathrm{g}) \quad$ Callus weight $(\mathrm{mg})$

$\begin{array}{ll}\text { Callus weight }(\mathrm{mg}) & 0.796\end{array}$

Callus ginsenosides

content (mg/g)

0.828

0.915 
Table 4.3. Ginsenosides content of stock roots and callus tissues of different ginseng lines.

\begin{tabular}{|c|c|c|c|c|c|c|c|c|c|c|c|c|c|c|c|c|}
\hline \multirow{3}{*}{$\begin{array}{c}\text { Ginseng } \\
\text { Lines }\end{array}$} & \multirow[b]{3}{*}{ sample } & \multicolumn{14}{|c|}{ Ginsenosides content $(\mathrm{mg} / \mathrm{g} \text { dry wt })^{\S}$} & \multirow[b]{3}{*}{ Weigth* } \\
\hline & & \multicolumn{7}{|c|}{ Stock roots } & \multicolumn{7}{|c|}{ Callus tissues } & \\
\hline & & Rg1 & $\mathrm{Re}$ & $\mathrm{Rb} 1$ & $\mathrm{Rc}$ & $\mathrm{Rb} 2$ & $\mathrm{Rd}$ & Total $^{6}$ & Rg1 & $\operatorname{Re}$ & $\mathrm{Rb} 1$ & $\mathrm{Rc}$ & $\mathrm{Rb} 2$ & $\mathrm{Rd}$ & Total $^{6}$ & \\
\hline \multirow[t]{6}{*}{ ML } & 1 & 1.40 & 16.19 & 14.74 & 5.83 & 0.21 & 2.77 & 41.14 & 0.85 & 4.14 & 8.81 & 0.41 & 0.54 & 0.29 & 15.04 & 135.58 \\
\hline & 2 & 0.05 & 6.74 & 19.18 & 2.65 & 0.13 & 0.53 & 29.28 & 0.51 & 4.11 & 7.22 & 0.21 & 0.45 & 0.18 & 12.68 & 131.60 \\
\hline & 3 & 2.43 & 10.28 & 10.12 & 5.94 & 0.26 & 0.70 & 29.73 & 0.88 & 4.74 & 6.99 & 0.31 & 0.52 & 0.34 & 13.78 & 125.12 \\
\hline & 4 & 2.31 & 16.06 & 14.35 & 6.12 & 0.23 & 0.80 & 39.87 & 0.76 & 4.62 & 7.52 & 0.51 & 0.48 & 0.29 & 14.18 & 135.54 \\
\hline & 5 & 1.70 & 9.01 & 17.19 & 1.93 & 0.10 & 0.31 & 30.24 & 0.92 & 4.51 & 7.01 & 0.35 & 0.48 & 0.37 & 13.64 & 123.82 \\
\hline & 6 & 1.58 & 15.18 & 15.46 & 5.27 & 0.24 & 2.49 & 40.22 & 0.62 & 5.59 & 7.56 & 0.29 & 0.42 & 0.27 & 14.75 & 134.88 \\
\hline \multirow[t]{6}{*}{ BLB } & 1 & 8.37 & 0.09 & 11.98 & 4.51 & 0.20 & 0.94 & 26.09 & 3.47 & 0.48 & 6.89 & 1.74 & 0.59 & 0.21 & 13.38 & 95.86 \\
\hline & 2 & 1.56 & 5.74 & 10.17 & 6.59 & 0.55 & 3.80 & 28.41 & 3.89 & 0.42 & 6.74 & 1.42 & 0.31 & 0.14 & 12.92 & 114.14 \\
\hline & 3 & 7.56 & 0.53 & 11.28 & 4.92 & 0.23 & 0.80 & 25.32 & 3.70 & 0.51 & 6.69 & 1.43 & 0.81 & 0.18 & 13.32 & 106.54 \\
\hline & 4 & 7.88 & 0.04 & 12.61 & 4.43 & 0.20 & 0.60 & 25.76 & 3.73 & 0.54 & 6.65 & 1.46 & 0.76 & 0.23 & 13.37 & 104.60 \\
\hline & 5 & 7.02 & 0.54 & 12.52 & 6.02 & 0.25 & 0.85 & 27.20 & 3.67 & 0.50 & 6.83 & 1.54 & 0.53 & 0.19 & 13.26 & 111.08 \\
\hline & 6 & 6.25 & 0.90 & 9.16 & 4.79 & 0.27 & 1.45 & 22.82 & 3.58 & 0.54 & 6.05 & 0.52 & 0.45 & 0.18 & 11.32 & 94.16 \\
\hline \multirow[t]{6}{*}{ STK } & 1 & 1.99 & 9.78 & 8.21 & 4.86 & 0.23 & 2.16 & 27.23 & 2.55 & 0.57 & 7.01 & 0.67 & 0.21 & 0.17 & 11.18 & 72.28 \\
\hline & 2 & 0.90 & 9.26 & 9.37 & 6.13 & 0.29 & 2.82 & 28.77 & 2.88 & 0.59 & 6.78 & 0.84 & 0.27 & 0.18 & 11.54 & 78.18 \\
\hline & 3 & 7.75 & 0.21 & 13.25 & 3.71 & 0.16 & 0.98 & 26.06 & 2.39 & 0.45 & 7.16 & 0.66 & 0.20 & 0.15 & 11.01 & 70.24 \\
\hline & 4 & 7.83 & 0.18 & 8.46 & 3.94 & 0.20 & 0.38 & 20.99 & 2.15 & 0.23 & 7.87 & 0.51 & 0.10 & 0.09 & 10.95 & 67.00 \\
\hline & 5 & 7.20 & 0.10 & 9.20 & 4.15 & 0.21 & 0.73 & 21.59 & 2.41 & 0.65 & 6.98 & 0.71 & 0.23 & 0.22 & 11.20 & 73.70 \\
\hline & 6 & 5.53 & 0.23 & 5.70 & 2.27 & 0.13 & 0.45 & 14.31 & 1.98 & 0.21 & 7.14 & 0.56 & 0.19 & 0.11 & 10.19 & 64.18 \\
\hline
\end{tabular}

$\S$ Values of individual ginsenoside are means of three measurements

${ }^{6}$ Sum of six preceding individual ginsenosides

* Dry weight (mg) of callus after 12 wks of culture in MS medium with $1.0 \mathrm{mg} \mathrm{l}^{-1} 2,4-\mathrm{D}$ and $0.1 \mathrm{mg} \mathrm{1^{-1 }}$ Kinetin. Weight values are means of 5 explant measurements per sample 
Table 4.4. Ginsenoside contents and profiles of roots and callus tissues of different ginseng lines. Values are means \pm SD of six replicates per line.

\begin{tabular}{|c|c|c|c|c|c|c|c|c|c|}
\hline & \multirow{2}{*}{$\begin{array}{c}\text { Ginseng } \\
\text { Lines } \\
\end{array}$} & \multicolumn{7}{|c|}{ Individual ginsenosides content (mg/g dry wt) } & \multirow{2}{*}{$\begin{array}{l}\text { Rg1/Re } \\
\text { profile }^{\mathbb{I I}}\end{array}$} \\
\hline & & $\operatorname{Rg} 1$ & $\operatorname{Re}$ & $\mathrm{Rb} 1$ & $\mathrm{Rc}$ & $\mathrm{Rb} 2$ & $\mathrm{Rd}$ & Total $^{6}$ & \\
\hline & & \multicolumn{7}{|c|}{ stock roots } & \\
\hline & BLB & $5.55 \pm 3.38$ & $2.20 \pm 1.95$ & $11.29 \pm 1.38$ & $5.21 \pm 0.89$ & $0.28 \pm 0.13$ & $1.41 \pm 1.21$ & $25.93 \pm 1.89$ & 1 \\
\hline & ML & $2.69 \pm 2.02$ & $11.13 \pm 6.23$ & $15.17 \pm 3.05$ & $4.62 \pm 1.84$ & $0.20 \pm 0.06$ & $1.27 \pm 1.07$ & $35.08 \pm 5.58$ & 2 \\
\hline & STK & $5.20 \pm 3.97$ & $3.29 \pm 1.72$ & $9.03 \pm 3.15$ & $4.18 \pm 2.25$ & $0.20 \pm 0.07$ & $1.25 \pm 1.00$ & $23.16 \pm 5.33$ & 1 \\
\hline \multirow[t]{5}{*}{ Significance } & & $*$ & $*$ & $*$ & NS & NS & NS & $*$ & \\
\hline & & \multicolumn{7}{|c|}{12 week old calli } & \\
\hline & BLB & $3.67 \pm 0.14$ & $0.50 \pm 0.04$ & $6.64 \pm 0.30$ & $1.35 \pm 0.42$ & $0.58 \pm 0.19$ & $0.19 \pm 0.03$ & $12.93 \pm 0.81$ & 1 \\
\hline & ML & $0.76 \pm 0.16$ & $4.62 \pm 0.54$ & $7.52 \pm 0.68$ & $0.35 \pm 0.10$ & $0.48 \pm 0.04$ & $0.29 \pm 0.29$ & $14.01 \pm 0.85$ & 2 \\
\hline & STK & $2.39 \pm 0.31$ & $0.45 \pm 0.19$ & $7.16 \pm 0.38$ & $0.66 \pm 0.12$ & $0.20 \pm 0.06$ & $0.15 \pm 0.05$ & $11.01 \pm 0.45$ & 1 \\
\hline Significance & & $*$ & $*$ & $*$ & $*$ & $*$ & $*$ & $*$ & \\
\hline
\end{tabular}

${ }^{6}$ Sum of six preceding individual ginsenosides

If 1 = high Rg1/low Re profile, $2=$ low Rg1/high Re profile

* Significant; NS = Non significant at $\alpha=0.05$, Tukey's test 
Table 4.5. Summary of primers used in this study.

\begin{tabular}{|l|l|c|c|c|}
\hline Primer & $5^{\prime}-3^{\prime}$ sequence & $\begin{array}{l}\text { Total } \\
\text { bands }\end{array}$ & $\begin{array}{l}\text { Polymorphic } \\
\text { bands }\end{array}$ & $\begin{array}{l}\text { \% of polymorphic } \\
\text { bands }\end{array}$ \\
\hline OPD-05 & TGAGCGGACA & 8 & 8 & 100.00 \\
\hline OPH-04 & GGAAGTCGCC & 5 & 4 & 80.00 \\
\hline OPH-05 & AGTCGTCCCC & 4 & 4 & 100.00 \\
\hline OPO-05 & TGGCGTCCTT & 9 & 9 & 100.00 \\
\hline OPAD-11 & CAATCGGGTC & 6 & 5 & 83.33 \\
\hline UBC-81 & GAGCACGGGG & 5 & 3 & 60.00 \\
\hline TOTAL & & 37 & 33 & 89.19 \\
\hline
\end{tabular}


Table 4.6. Distance matrix among individual roots within ginseng lines based on RAPD markers. Distance values based on Nei's (1978) unbiased minimum distance.

\begin{tabular}{|c|c|c|c|c|c|c|c|c|c|c|c|c|c|c|c|c|c|c|}
\hline $\begin{array}{l}\text { Ginseng } \\
\text { Line }\end{array}$ & ML-1 & ML-2 & ML-3 & ML-4 & ML-5 & ML-6 & BLB-1 & BLB-2 & BLB-3 & BLB-4 & BLB-5 & BLB-6 & BLB-1 & BLB-2 & BLB-3 & BLB-4 & BLB-5 & BLB-6 \\
\hline ML-1 & 0.0000 & & & & & & & & & & & & & & & & & \\
\hline ML-2 & 0.7576 & 0.0000 & & & & & & & & & & & & & & & & \\
\hline ML-3 & 0.7273 & 0.1515 & 0.0000 & & & & & & & & & & & & & & & \\
\hline ML-4 & 0.6364 & 0.3636 & 0.2727 & 0.0000 & & & & & & & & & & & & & & \\
\hline ML-5 & 0.5758 & 0.4242 & 0.3333 & 0.0606 & 0.0000 & & & & & & & & & & & & & \\
\hline ML-6 & 0.6364 & 0.3636 & 0.2727 & 0.0606 & 0.1212 & 0.0000 & & & & & & & & & & & & \\
\hline BLB-2 & 0.6970 & 0.4242 & 0.2727 & 0.3636 & 0.4242 & 0.4242 & 0.0303 & 0.0000 & & & & & & & & & & \\
\hline BLB-3 & 0.7576 & 0.3636 & 0.2727 & 0.3636 & 0.4242 & 0.4242 & 0.0303 & 0.0606 & 0.0000 & & & & & & & & & \\
\hline BLB-4 & 0.7576 & 0.2424 & 0.1515 & 0.3030 & 0.3636 & 0.3636 & 0.1515 & 0.1818 & 0.1818 & 0.0000 & & & & & & & & \\
\hline BLB-5 & 0.6970 & 0.2424 & 0.1515 & 0.3030 & 0.3636 & 0.3636 & 0.3333 & 0.3030 & 0.3030 & 0.1818 & 0.0000 & & & & & & & \\
\hline BLB-6 & 0.7273 & 0.0303 & 0.1818 & 0.3333 & 0.3939 & 0.3333 & 0.4242 & 0.4545 & 0.3939 & 0.2727 & 0.2727 & 0.0000 & & & & & & \\
\hline STK-1 & 0.7273 & 0.2121 & 0.1818 & 0.3939 & 0.4545 & 0.3939 & 0.2424 & 0.2727 & 0.2121 & 0.2121 & 0.3333 & 0.2424 & 0.0000 & & & & & \\
\hline STK-2 & 0.7576 & 0.3636 & 0.2727 & 0.4242 & 0.4848 & 0.4848 & 0.0909 & 0.1212 & 0.1212 & 0.1212 & 0.3030 & 0.3939 & 0.2727 & 0.0000 & & & & \\
\hline STK-3 & 0.6970 & 0.4242 & 0.2727 & 0.3636 & 0.4242 & 0.4242 & 0.1515 & 0.1212 & 0.1212 & 0.2424 & 0.3030 & 0.3939 & 0.2727 & 0.1212 & 0.0000 & & & \\
\hline STK-4 & 0.6970 & 0.1818 & 0.0303 & 0.3030 & 0.3636 & 0.3030 & 0.3333 & 0.3030 & 0.3030 & 0.1818 & 0.1818 & 0.2121 & 0.2121 & 0.3030 & 0.3030 & 0.0000 & & \\
\hline STK-5 & 0.6970 & 0.3030 & 0.2121 & 0.3636 & 0.4242 & 0.4242 & 0.3333 & 0.3030 & 0.3030 & 0.2424 & 0.0606 & 0.3333 & 0.3333 & 0.3030 & 0.3030 & 0.1818 & 0.0000 & \\
\hline
\end{tabular}

Values in bold indicate minimum and maximum distances 
Table 4.7. Distance matrix of three ginseng lines based on RAPD markers. Distance values based on Nei's (1978) unbiased minimum distance.

\begin{tabular}{lccc}
\hline Ginseng line & ML & BLB & STK \\
\hline ML & 0.0000 & & \\
BLB & 0.1115 & 0.0000 & \\
STK & 0.1260 & 0.0260 & 0.0000 \\
\hline
\end{tabular}




\section{CHAPTER 5}

General Summary 
American ginseng (Panax quinquefolius L.) is a perennial understory herb that is highly renowned for its medicinal properties (Duke 1989; Persons 1994). Like many other medicinal plants worldwide, $P$. quinquefolius is mainly collected from the wild. And because of its growing demand abundance of wild populations is threatened with over harvesting (Robbins 1998). In West Virginia, P. quinquefolius is available both in cultivation and in the wild. Even though P. quinquefolius occurs in all 55 counties in West Virginia (WVDOF 2008), patterns of its harvesting and cultivation vary across the state. For instance most of wild harvesting occurs in the southern region in comparison to other regions, whereas large scale cultivation occurs in the northern region of the state. How these varied levels of human influence have shaped the genetic diversity and population structure of $P$. quinquefolius in West Virginia is relatively unexplored. Unlike other medicinal plants that are mostly harvested for their leaf materials, P. quinquefolius is harvested for its root material. The root of $P$. quinquefolius is unique because it has a tendency to branch into different morphotypes, and this variation in root shapes influences the perceived quality and hence value of $P$. quinquefolius roots in the market (Kim 2005; Roy et al. 2003, 2008).

The objectives of this study were: first, assess the genetic diversity and population structure of $P$. quinquefolius growing in West Virginia and compare it to populations from Pennsylvania and Wisconsin. Second, determine the effects of anthropogenic activities (harvesting pressure and cultivation intensity) on genetic diversity of $P$. quinquefolius growing in West Virginia. Third, compare the variation in concentrations and profiles of ginsenosides among three different root morphotypes (BLB, ML, and 
STK) and explore possible economic implications to $P$. quinquefolius marketing. Fourth, assess genetic differences among these root morphotypes and evaluate how explants obtained from these roots respond to in vitro callus initiation, growth and ginsenosides production.

Overall, results from 26 populations (14 wild and 12 cultivated) evaluated in this study showed higher levels of genetic diversity in cultivated than wild populations. Most of the variation in cultivated populations was attributed to individuals within populations, whereas in wild populations greater variance was partitioned to differentiation among populations. This finding is in agreement with other studies on $P$. quinquefolius (Schluter and Punja 2002; Mooney 2007). Cultivated populations typically have a higher degree of gene flow among populations facilitated by greater movement of seeds between growers as opposed to limited gene flow among wild populations mainly caused by isolation of populations due to fragmented habitats.

In general, West Virginia populations exhibited lower indices of genetic diversity compared to populations from Pennsylvania and Wisconsin. Low genetic diversity in West Virginia populations particularly for wild populations could be a consequence of harvesting pressure associated with the long history of wild $P$. quinquefolius collection in the state. There was no significant difference in genetic diversity between cultivated and wild $P$. quinquefolius populations in West Virginia. Although this was unexpected, at least it can be explained by high level of genetic similarity observed between wild and cultivated populations from low cultivation intensity regions which could be due to use of 
seeds collected from the wild to start $P$. quinquefolius cultivation in most small scale farms.

There was a significant effect of cultivation intensity on genetic diversity of $P$. quinquefolius in West Virginia. Populations from high cultivation intensity regions had higher genetic diversity indices compared to those from low cultivation intensity regions. Likewise, among population differentiation was lower in high cultivation intensity regions but high in low cultivation intensity regions. This implies that high cultivation intensity consequently leads to increase in genetic diversity among individuals within populations because of broader sourcing of seeds to meet the needs of large scale $P$. quinquefolius farming. With regard to harvest pressure, populations from low harvest pressure regions had higher diversity indices than populations from high harvest pressure regions, although these genetic diversity indices were not statistically significant. Nonetheless, lower diversity indices observed in high harvest pressure region further reaffirms the detrimental effects of harvest pressure on $P$. quinquefolius populations (Cruse-Sanders and Hamrick 2004; Cruse-Sanders et al. 2005).

Comparison of ginsenosides content among different root morphotypes (ML, BLB and STK) of $P$. quinquefolius showed significant variation in concentration and profiles of individual and total ginsenosides. Roots of ML morphotype had higher total ginsenosides content than roots of BLB or STK morphotypes. However there was no significant difference in total ginsenoside content between BLB and STK roots. The concentrations of ginsenosides Re and Rb1 was higher in roots of ML morphotypes 
compared to roots of BLB and STK morphotypes. However ginsenoside Rg1 was lower in roots of ML morphotype. Roots of ML morphotype exclusively exhibited low Rg1/high Re profile, whereas roots of BLB and STK morphotypes exhibited both low $\operatorname{Rg} 1 /$ high Re, and high $\operatorname{Rg} 1 /$ low Re profiles. Basing root quality on total ginsenosides alone, then roots of ML morphotypes could be regarded to be of a higher quality than those of BLB or STK morphotypes. Tabulation of frequency distribution of morphotypes in both field and greenhouse production showed that ML and STK roots were the least and the most abundant morphotypes respectively. Variation in root morphotypes was observed in greenhouse grown roots as young as 3 months old. Given that physical and environmental factors that may influence root shape were held uniform in the greenhouse, the observed variation on root shape could be an influence of underlying genetics. However, further research using robust genomic tools will be needed to explore genetic linkage to root morphology in $P$. quinquefolius. This will present breeding opportunities for this species.

Currently there are no cultivars for P. quinquefolius (Schluter and Punja 2002), and this has been attributed to the general lack of breeding direction for medicinal plants (Hong et al. 2005). However, in the light of various reports on pharmacological activities of individual ginsenosides, breeding targets for P. quinquefolius are becoming apparent. For instance, $\mathrm{Re}$ is reported to be effective against diabetes and helps lower cholesterol (Attele et al. 2002; Xie et al. 2005; Cho et al. 2006), therefore cultivars with high Re levels (ML roots) could be desirable. On the other hand, $\mathrm{Rg} 1$ has been reported to have 
neuroprotective properties (Liao et al. 2002), so cultivars with high concentration of $\operatorname{Rg} 1$ (BLB and STK morphotypes) could be targeted.

In the light of the above findings, economical analysis was conducted to explore if sorting of harvested roots to respective morphotypes before selling could leverage returns for the grower. Result from economic analysis showed that, under the current production system (no selected cultivars), sorting roots to different morphotypes could result in revenue increase. The level of return however will be dependent upon the cost of sorting and price premium mark up the grower will assign to high quality roots (ML). However if sorting will result in lowering the price value of the other two root morphotypes (BLB and STK), then even at the lowest sorting cost this strategy will result in a net loss when compared to selling roots as a mixed batch. This is simply because of the low abundance of ML morphotype roots in the population. However, substantial economic gain is expected if successful breeding and production of high quality root morphotype is attained in future.

Lastly, this study assessed how explants derived from different root morphotypes of $P$. quinquefolius responded to in vitro callus induction, growth and ginsenosides production. Data from this study showed that explants from different root morphotypes exhibited different response to callus induction, callus growth and ginsenosides production. There was a positive and significant correlation between ginsenosides content of stock plants and that of callus tissues, and between callus growth and ginsenosides content of stock plants. Explants from stock plants with high total 
ginsenosides content induced callus faster, were prolific in growth, and accumulated more total ginsenoside in their callus tissues than explants from stock plants with low ginsenosides content. This could be one of the reasons for the observed fluctuation of in vitro ginsenosides production in previous studies in the genus Panax (Wu and Zhong 1999) and could be an important consideration for in vitro production of bioactive components of any medicinal plant.

RADP analysis revealed some genetic differences among root morphotypes. Based on molecular distances of their RAPD profiles, BLB and STK roots grouped into the same cluster and separate from ML roots. Also data from HPLC analysis showed that there was a significant variation in ginsenosides profiles and content among root morphotypes of stock plants. However, a specific link between a particular DNA band or banding pattern and a given profile or abundance of ginsenosides was not apparent. However, this line of research will need to be explored further in a bid to identify DNA markers that can be used to identify plants with desired ginsenosides profiles. Those markers can then be used to facilitate breeding of this important medicinal plant. 


\section{Literature Cited}

Attele, A. S., Y.P. Zhou, J.T. Xie, J.A. Wu, L. Zhang, L. Dey, W. Pugh, P.A. Rue, K.S. Polonsky, and C.S. Yuan. 2002. Antidiabetic effects of Panax ginseng berry extract and the identification of an effective component. Diabetes 51: 1851-1858.

Cho, W.C.S., W-S. Chung, S.K.W. Lee, A.W.N. Leung, C.H.K. Cheng, and K.K.M. Yue. 2006. Ginsenoside Re of Panax ginseng possesses significant antioxidant and antihypelipidemic efficacies in streptozotocin-induced diabetic rats. European Journal of Pharmacology 550: 173-179.

Duke, J. 1989. Ginseng: A concise handbook. Reference Publication, Inc. Algonac. Mississippi. USA.

Kim, H., B. Sung, J. Park, C. Sim, Y. Kim, and S. Lee. 2005. Application of neutron radiography and tomography for analysis of root morphology of growing ginseng. Proceedings of the International Symposium on Research Reactor and Neutron Science - In Commemoration of the $10^{\text {th }}$ Anniversary of HANARO- Daejeon, Korea.

Hong, D. Y. Q., A. J. Lau, C. L. Yeo, X. K. Liu, C. R. Yang, H. L. Koh, and Y. Hong. 2005. Genetic diversity and variation of saponin contents in Panax notoginseng roots from a single farm. Journal of Agricultural and Food Chemistry 53: 84608467.

Liao, B., H. Newmark, and R. Zhou. 2002. Neuroprotective effects of ginseng total saponin and ginsenoside Rb1 and Rg1 on spinal code neurons in vitro. Experimental Neurology 173: 224-234. 
Mooney, H.E. 2007. Genetic and evolutionary consequences of harvest in American ginseng, Panax quinquefolius L. (Araliaceae). Ph.D. dissertation. West Virginia University, Morgantown, West Virginia.USA.

Persons, W. S. 1994. American ginseng: Green Gold. Bright Mountain Books, Inc. Asheville, North Carolina, USA.

Robbins, C. S. 1998. American ginseng: The root of North America's medicinal herb trade. TRAFFIC North America Report. Number B347, Washington, D.C. USA.

Roy, R.C., R. Grohs, and R.D. Reeleder. 2003. A method for the classification by shape of dried roots of ginseng (Panax quinquefolius L.). Canadian Journal of Plant Science 83: 955-958.

Roy, R.C., B.R. Ball Coelho, R.D. Reeleder, A.J. Bruin, R. Grohs, P.H. White, and B.B. Capell. 2008. Effect of planting bed shape, mulch and soil density on root yield and shape in North American ginseng (Panax quinquefolius L.). Canadian Journal of Plant Science 88 (5): 937-949.

Schluter, C. and Z. K. Punja. 2002. Genetic diversity among natural and cultivated field populations and seed lots of American ginseng (Panax quinquefolius L.) in Canada. International Journal of Plant Science 163 (3): 427-439.

Wu, J., and J.-J. Zhong. 1999. Production of ginseng and its bioactive compounds in plant cell culture: current technological and applied aspects. Journal of Biotechnology 68: 89-99.

WVDOF. 2008. http://www.wvforestry.com/ginseng.cfm?menucall=ginseng. Accessed April 22, 2008. 
Xie, J-T., S.R. Mehendale, X. Li, R. Quigg, X. Wang, C-Z. Wang, J.A. Wu, H.H. Aung, P.A. Rue, G.I. Bell, and C-S. Yuan. 2005. Anti-diabetic effect of ginsenoside Re in ob/ob mice. Biochimica et Biophysica Acta 1740 (3): 319-325. 